DCPT- $11 / 31$

\title{
The overarching finite symmetry group of Kummer surfaces in the Mathieu group $M_{24}$
}

\author{
Anne Taormina* and Katrin Wendland ${ }^{\dagger}$
}

\author{
${ }^{*}$ Centre for Particle Theory \& Department of Mathematical Sciences, \\ Durham University, Science Laboratories, South Road, Durham, DH1 3LE, U.K. \\ ${ }^{\dagger}$ Mathematics Institute, Albert-Ludwigs-Universität Freiburg, \\ Eckerstraße 1, Freiburg im Breisgau, D-79104, Germany.
}

\begin{abstract}
In view of a potential interpretation of the role of the Mathieu group $M_{24}$ in the context of strings compactified on K3 surfaces, we develop techniques to combine groups of symmetries from different K3 surfaces to larger 'overarching' symmetry groups. We construct a bijection between the full integral homology lattice of K3 and the Niemeier lattice of type $A_{1}^{24}$, which is simultaneously compatible with the finite symplectic automorphism groups of all Kummer surfaces lying on an appropriate path in moduli space connecting the square and the tetrahedral Kummer surfaces. The Niemeier lattice serves to express all these symplectic automorphisms as elements of the Mathieu group $M_{24}$, generating the 'overarching finite symmetry group' $\left(\mathbb{Z}_{2}\right)^{4} \rtimes A_{7}$ of Kummer surfaces. This group has order 40320 , thus surpassing the size of the largest finite symplectic automorphism group of a K3 surface by orders of magnitude. For every Kummer surface this group contains the group of symplectic automorphisms leaving the Kähler class invariant which is induced from the underlying torus. Our results are in line with the existence proofs of Mukai and Kondo, that finite groups of symplectic automorphisms of K3 are subgroups of one of eleven subgroups of $M_{23}$, and we extend their techniques of lattice embeddings for all Kummer surfaces with Kähler class induced from the underlying torus.
\end{abstract}

\section{Introduction}

$M_{24}$ is the largest in a family of five sporadic groups - amongst the 26 appearing in the classification of finite simple groups - that has rekindled interest in the mathematical physics community following an intriguing remark published by Eguchi, Ooguri and Tachikawa 1. This remark stems from an expression for the elliptic genus of a K3 surface that uses knowledge of 2-dimensional $N=4$ superconformal field theory and Witten's construction of elliptic genera [2, 3. That the K3 elliptic genus, which is a weak Jacobi form of weight 0 and index 1, may be expanded in a linear combination of $N=4$ superconformal characters is not surprising. Indeed in the context of superstring theory, it has long been established that

\footnotetext{
*anne.taormina@durham.ac.uk

${ }^{\dagger}$ katrin.wendland@math.uni-freiburg.de
} 
compactification on a K3 surface, which is a hyperkähler manifold, yields a world-sheet theory that is invariant under $N=4$ superconformal transformations. The K3 elliptic genus may be calculated as a specialisation of the corresponding partition function, which is a sesquilinear expression in the $N=4$ characters [4].

What is surprising and remains to be fully understood, is that the coefficients of the non-BPS $N=4$ characters in the elliptic genus decomposition coincide with the dimensions of some irreducible and reducible representations of the sporadic group $M_{24}$. Actually, on the basis of the information encoded in the K3 elliptic genus alone, the dimensions could be those of representations of the Mathieu group $M_{23}$, the stabilizer in $M_{24}$ of an element in the set $\mathcal{I}=\{1,2, \ldots, 24\}$, when viewing $M_{24}$ as the group of permutations of 24 elements preserving the extended binary Golay code $\mathcal{G}_{24}$. It turns out that this Mathieu group information is encoded in the K3 elliptic genus in the form of a weakly holomorphic mock modular form of weight $\frac{1}{2}$ on $S L(2, \mathbb{Z})$, suggesting the existence of a "Mathieu Moonshine" phenomenon [5, 7, 6, 8].

Yet, an interpretation of the appearance of $M_{24}$ as a symmetry group within a theory of strings compactified on K3 is lacking. One difficulty is that the $M_{24}$ sporadic group does not act in a conventional way on the string states. Rather, the symmetry manifests itself when a specific subset of BPS states is considered. Another particularity is that by nature, the elliptic genus is an invariant on each irreducible component of the moduli space of $N=(2,2)$ superconformal field theories. The moduli space of superconformal theories on K3 is such an irreducible component. Hence, the $M_{24}$-information the elliptic genus carries is unaltered when surfing the K3 moduli space between generic points and isolated points with enhanced symmetry, of which Gepner models are the main examples. This suggests that $M_{24}$, in a sense yet to be uncovered, 'overarches' the symmetries associated with all superconformal field theories in the K3 moduli space.

Although we have, at this stage, little new to say regarding the interpretation of an $M_{24}$-action within the framework of strings compactified on K3 surfaces, we show here how an overarching symmetry, smaller than $M_{24}$, emerges when considering the finite groups of symplectic automorphisms of all K3 surfaces of a particular type. Namely, we consider symmetry groups of Kummer surfaces, by which we mean the groups of those symplectic automorphisms that preserve the Kähler class induced by that of the underlying complex torus. The results presented in this work should be regarded as an attempt to set the scene for further investigations pinning down an 'overarching' $M_{24}$-action as alluded to above. We revisit the results obtained by Mukai in [9], stating that any finite group of symplectic automorphisms of a K3 surface is isomorphic to a subgroup of the Mathieu group $M_{23}$ which has at least 5 orbits on the set $\mathcal{I}$ of 24 elements. We develop a technique which allows to combine the symmetry groups of several different Kummer surfaces. With this technique, we obtain the group $\left(\mathbb{Z}_{2}\right)^{4} \rtimes A_{7}$ of order $2^{7} \cdot 3^{2} \cdot 5 \cdot 7=40320$, which is a maximal subgroup of $M_{23}$. This group contains as proper subgroups all symplectic automorphism groups of Kummer surfaces preserving the induced Kähler class. In this sense, we find an 'overarching' symmetry group of all Kummer surfaces. In [10], Kondo rederives Mukai's result, using ingenious lattice techniques. The framework we set up here is in this spirit. We use the Niemeier lattice of type $A_{1}^{24}$, denoted $N$ hereafter, as a device which for all Kummer surfaces encodes the action of the symmetry groups as automorphisms of the extended binary Golay code, represented by permutations on 24 elements belonging to $M_{24}$. This encoding is linked to the construction of a bijection $\theta$ between the full integral homology lattice $H_{*}(X, \mathbb{Z})$ of a Kummer surface $X$ and the negative definite version of the Niemeier lattice $N$, denoted $N(-1)$. Here, the lattice $H_{*}(X, \mathbb{Z})$ is identified with the standard unimodular lattice of the appropriate signature by an isometry which is induced by the Kummer construction and thus is naturally fixed and compatible with all Kummer surfaces. Although in general, the bijection $\theta$ between $H_{*}(X, \mathbb{Z})$ and $N(-1)$ depends on the 
complex structure and Kähler class of the Kummer surface considered, we are able to construct a unique bijection $\Theta$, up to a few choices of signs, which for two specific Kummer surfaces denoted $X_{0}$ and $X_{D_{4}}$ yields an action of their distinct symmetry groups, $\mathcal{T}_{64}$ and $\mathcal{T}_{192}$ respectively, on the same Niemeier lattice $N$. Moreover, $\Theta$ is compatible with the generic symmetry group $G_{t} \cong\left(\mathbb{Z}_{2}\right)^{4}$ of Kummer surfaces. In fact, we argue that we are naturally working in a smooth connected cover $\widetilde{\mathcal{M}}_{h k}$ of the moduli space of hyperkähler structures on K3. Namely, the symplectic automorphism group of a K3 surface which preserves a given Kähler class solely depends on the hyperkähler structure determined by the invariant complex structure and Kähler class. The choice of an isometric identification of $H_{*}(X, \mathbb{Z})$ with a fixed standard lattice amounts to the transition to the smooth connected cover $\widetilde{\mathcal{M}}_{h k}$ of the moduli space. We show that our bijection $\Theta$ is compatible with the symmetry group of all K3 surfaces along a particular path which connects $X_{0}$ and $X_{D_{4}}$ in $\overline{\mathcal{M}}_{h k}$. Indeed, there exists such a path consisting of Kummer surfaces, all of whose symmetry groups away from beginning and end of the path restrict to the generic one, $G_{t} \cong\left(\mathbb{Z}_{2}\right)^{4}$. Our bijection $\Theta$ therefore effectively reveals the presence of a larger, overarching and somehow hidden symmetry that could manifest itself in an unsuspected way within string theories compactified on K3 surfaces. In our eyes, the significance of our result is that it provides a framework allowing to pin down overarching symmetry groups that transcend known symmetry groups of K3 surfaces given by distinct points in our moduli space. In the specific case of $\widetilde{\mathcal{M}}_{h k}$, one ultimately wishes the overarching symmetry group to be $M_{24}$, in accordance with the information encoded in the K3 elliptic genus. Although the overarching symmetry group $\left(\mathbb{Z}_{2}\right)^{4} \rtimes A_{7}$ we find is two orders of magnitude larger than the biggest finite symplectic automorphism group of a K3 surface, it is still by orders of magnitude smaller than the Mathieu group $M_{24}$. In order to obtain $M_{24}$, further techniques and different lattices are required, and this is beyond the scope of the present work.

Mathematically, one of our main results is the following theorem, which holds for all Kummer surfaces and which we prove in section 3, see theorem 3.3.7.

Theorem. Let $X$ denote a Kummer surface with underlying torus $T$, equipped with the complex structure and Kähler class which are induced from $T$. By $E_{\vec{a}}, \vec{a} \in \mathbb{F}_{2}^{4}$, we denote the classes in $H_{2}(X, \mathbb{Z})$ obtained in the Kummer construction by blowing up the 16 singular points of $T / \mathbb{Z}_{2}$. Let $G$ denote the group of symplectic automorphisms of $X$ which preserve the Kähler class. With $L_{G}=\left(H_{*}(X, \mathbb{Z})^{G}\right)^{\perp} \cap H_{*}(X, \mathbb{Z})$ and $v_{0} \in H_{0}(X, \mathbb{Z}), v \in H_{4}(X, \mathbb{Z})$ such that $\left\langle v_{0}, v\right\rangle=1$, and $e:=\frac{1}{2} \sum_{\vec{a} \in \mathbb{F}_{2}^{4}} E_{\vec{a}}$, the lattice $M_{G}(-1)$ with

$$
M_{G}:=L_{G} \oplus \operatorname{span}_{\mathbb{Z}}\left\{e, v_{0}-v\right\}
$$

can be primitively embedded in the Niemeier lattice $N$ of type $A_{1}^{24}$.

The theorem generalizes Kondo's proof [10] to Mukai's theorem [9] in the following sense: For a K3 surface $X$, Kondo proves that there exists some Niemeier lattice $\widetilde{N}$, such that the lattice $L_{G}$ can be primitively embedded in $\widetilde{N}(-1)$. Although Kondo primitively embeds a lattice $L_{G} \oplus\langle-2\rangle$, the additional direction $\langle-2\rangle$ is not identified geometrically within $H_{*}(X, \mathbb{Z})$. Our first contribution thus is the natural geometric interpretation $\langle-2\rangle=\operatorname{span}_{\mathbb{Z}}\left\{v_{0}-v\right\}$. Second, for every Kummer surface with induced Kähler class, our theorem shows that in Kondo's proof, the special Niemeier lattice $N$ of type $A_{1}^{24}$ can always be used 1 . Third, the lattice $M_{G}$ which we embed contains Kondo's lattice $L_{G}$ and obeys $\mathrm{rk}\left(M_{G}\right)=\operatorname{rk}\left(L_{G}\right)+2$. This embedding is the restriction to $M_{G}$ of the bijection $\Theta$ between $H_{*}(X, \mathbb{Z})$ and $N(-1)$ described above. The construction of such a bijection is an application of the "gluing techniques" due to Nikulin [11, 12 that build on previous work by Witt [13] and Kneser [14]. Our specific bijection $\Theta$ isometrically identifies two different primitive sublattices $M_{\mathcal{T}_{192}}$ and $M_{\mathcal{T}_{64}}$ in $H_{*}(X, \mathbb{Z})$ with

\footnotetext{
${ }^{1}$ This nicely ties in with Mukai's result [10, Appendix] that there exists a symplectic action on the special Niemeier lattice $N$ for each group $G$ in Mukai's classification.
} 
their images. Furthermore, we force these embeddings to be equivariant with respect to the two corresponding symmetry groups $G=\mathcal{T}_{192}$ and $G=\mathcal{T}_{64}$, thus obtaining natural actions of these two groups on $N$. This allows us to view $N$ as a device which carries both these group actions, and in fact the actions of all symmetry groups along an appropriate path in moduli space, which combine to the action of a larger, overarching group. Interestingly, the resulting group already gives the overarching symmetry group of all Kummer surfaces. Since our techniques confine us to the study of symmetries of Kummer surfaces, the fact that we can generate the entire overarching symmetry group of Kummer surfaces may very well mean that on transition to more general techniques, employing the Leech lattice instead of the Niemeier lattice $N$, say, one could obtain the entire group $M_{24}$. We are currently working on such a generalisation.

Our techniques were originally designed to study non-classical symmetries of superconformal field theories compactified on K3 surfaces, particularly to develop a device which could maybe distinguish between those symmetries which play a role for "Mathieu Moonshine" and those that don't. Indeed2, in the beautiful work [15] it is proved that the symmetry groups of superconformal field theories on K3 in general are not subgroups of $M_{24}$, but instead that they are all contained in the Conway group $\mathrm{Co}_{1}$. Our findings could in fact indicate that the classical symmetries of K3 are the only ones that play a role for "Mathieu Moonshine".

The paper is organised as follows.

Section 2 starts with a short review of Nikulin's lattice gluing prescription and illustrates it in two cases of direct interest to us: the gluing of the Kummer lattice to the lattice stemming from the underlying torus of a given Kummer surface, including an extension of this technique to recover the full integral homology, as well as the reconstruction of the Niemeier lattice $N$ associated with the root lattice of $A_{1}^{24}$ through the gluing of two different pairs of sublattices. This construction of the integral K3-homology provides an isometric identification of the homology lattice $H_{*}(X, \mathbb{Z})$ with a standard lattice which is induced by the Kummer construction and which is used throughout this work. Built in this section is proposition 2.3.3, stating and proving that the Niemeier lattice $N$ possesses a rank 16 primitive sublattice $\widetilde{\Pi}$ which, up to a total reversal of signature, is isometric to the Kummer lattice $\Pi$. That the primitive embedding of the Kummer lattice in $N(-1)$ is unique up to automorphisms of $N$ is proven in proposition 2.3.5. Moreover, an explicit realisation of the primitive sublattice $\widetilde{\Pi}$ that proves extremely useful in our construction is provided via the map (2.14). These properties of the Niemeier lattice $N$ of type $A_{1}^{24}$, to our knowledge, had not been noticed before.

After reviewing basic albeit crucial aspects of complex structures, Kähler forms and symplectic automorphisms of K3 surfaces, we argue that by our assumptions, we are in fact working on the smooth connected cover $\widetilde{\mathcal{M}}_{h k}$ of the moduli space of hyperkähler structures on K3. Next, after providing a summary of the results obtained by Mukai and Kondo that are important for our analysis, Section 3 generalizes Kondo's results to the full integral homology lattice to fit our purpose. Moreover, it introduces the lattice $M_{G} \supsetneq L_{G}$ that is so crucial for our construction, and proves that this lattice, after a total reversal of signature, may be embedded primitively in the Niemeier lattice $N$, as stated in theorem 3.3.7 and as discussed above.

Section 4 builds on the previous sections to bring to light the overarching symmetry that is at the centre of this work. This is achieved by constructing a linear bijection $\Theta$ that extends the isometry between the Kummer lattice and $\widetilde{\Pi}(-1)$ to the full integral homology $H_{*}(X, \mathbb{Z})$, which as mentioned above is explicitly identified with a fixed standard lattice by means of the Kummer construction, and $N(-1)$. We start in subsection 4.1 with the discussion of the translational automorphism group that is a normal subgroup of all symplectic automorphism

\footnotetext{
${ }^{2}$ We are grateful to Rob Curtis for explaining to us in April 2010 that the well-known symmetry group $C_{4}^{3} \rtimes S_{4}$ of the Gepner model $(2)^{4}$ is not a subgroup of $M_{24}$.
} 
groups of Kummer surfaces which preserve the induced Kähler class, and show how its action on $X$ induces an action of $\left(\mathbb{Z}_{2}\right)^{4}$ on $N$. Subsections 4.2 and 4.3 are dedicated to the tetrahedral Kummer surface $X_{D_{4}}$ with symmetry group $\mathcal{T}_{192} \subset M_{24}$, and to the Kummer surface $X_{0}$ obtained from the square torus $T_{0}$, whose symmetry group is $\mathcal{T}_{64} \subset M_{24}$. In subsection 4.4 we prove that these two Kummer surfaces enjoy the same 'overarching' linear bijection $\Theta: H_{*}(X, \mathbb{Z}) \longrightarrow N(-1)$, leading to the concept of overarching symmetry. Moreover, we construct a smooth path from $X_{0}$ to $X_{D_{4}}$ in the smooth connected cover $\widetilde{\mathcal{M}}_{h k}$ of the moduli space of hyperkähler structures on K3 such that the symmetry group of every K3 surface along the path is compatible with $\Theta$.

We end by giving our conclusions and interpretations of our results, including some evidence for the expectation that our techniques may eventually show Mathieu moonshine to be linked to the group $M_{24}$ rather than $M_{23}$. Appendix $\AA$ provides a brief review of some of the notions and techniques we borrowed from group theory in the course of our work, while appendix B gathers notations for most lattices introduced and used in the main body of the text.

\section{Lattices: Gluing and primitive embeddings}

In this work, we investigate certain finite groups of symplectic automorphisms of Kummer surfaces in terms of subgroups of the Mathieu group $M_{24}$. As we explain below, due to the Torelli Theorem 3.1.1 for K3 surfaces, on the one hand, and the natural action of $M_{24}$ on the Niemeier lattice $N$ of type $A_{1}^{24}$ by proposition 2.3.2, on the other hand, both group actions are naturally described in terms of lattice isometries. Therefore, some of the main techniques of our work rest on lattice constructions, which shall be recalled in this section. As a guide to the reader, table 1 in appendix B lists a number of lattices that we introduce in this section and that we continue to use throughout this work.

Let us first fix some standard terminology (see for example [12, 16]):

Definition 2.0.1 Consider a d-dimensional real vector space $V$ with scalar product $\langle\cdot, \cdot\rangle$ of signature $\left(\gamma_{+}, \gamma_{-}\right)$.

1. A lattice $\Gamma$ in $V$ is a free $\mathbb{Z}$-module $\Gamma \subset V$ together with the symmetric bilinear form induced by $\langle\cdot, \cdot\rangle$ on $\Gamma$. Its DISCRIMINANT $\operatorname{disc}(\Gamma)$ is the determinant of the associated bilinear form on $\Gamma$. The lattice $\Gamma$ is NON-DEGENERATE if $\operatorname{disc}(\Gamma) \neq 0$, and it is UNIMODUlar if $|\operatorname{disc}(\Gamma)|=1$. By $\Gamma(N)$ with $N$ an integer we denote the same $\mathbb{Z}$-module as $\Gamma$, but with bilinear form rescaled by a factor of $N$.

2. A lattice $\Gamma$ in $V$ is INTEgRal if its bilinear form has values in $\mathbb{Z}$ only. It is EVEN if the associated quadratic form has values in $2 \mathbb{Z}$ only.

3. If $\Gamma$ is a non-degenerate even lattice in $V$, then there is a natural embedding $\Gamma \hookrightarrow$ $\Gamma^{*}=\operatorname{Hom}(\Gamma, \mathbb{Z})$ by means of the bilinear form on $\Gamma$. Thus there is an induced $\mathbb{Q}$ valued symmetric bilinear form on $\Gamma^{*}$. The DISCRIMINANT FORM $q_{\Gamma}$ of $\Gamma$ is the map $q_{\Gamma}: \Gamma^{*} / \Gamma \rightarrow \mathbb{Q} / 2 \mathbb{Z}$ together with the symmetric bilinear form on the DISCRIMINANT GROUP $\Gamma^{*} / \Gamma$ with values in $\mathbb{Q} / \mathbb{Z}$, induced by the quadratic form and symmetric bilinear form on $\Gamma^{*}$, respectively.

4. A sublattice $\Lambda \subset \Gamma$ of a lattice $\Gamma$ in $V$ is a PRimitive sublattice if $\Gamma / \Lambda$ is free.

By this definition, we can view every sublattice $\Lambda \subset \Gamma$ of $\Gamma \subset V$ as a lattice in the vector space $\Lambda \otimes \mathbb{R}$ with the induced quadratic form. In fact, since $V=\Gamma \otimes \mathbb{R}$ for non-degenerate $\Gamma$, we can dispense with the mention of the vector space that any of our lattices is constructed in. Note that $\Lambda$ is a primitive sublattice of $\Gamma$ if and only if $\Lambda=(\Lambda \otimes \mathbb{Q}) \cap \Gamma$. Moreover, for 
every non-degenerate even lattice $\Gamma$ we have $|\operatorname{disc}(\Gamma)|=\left|\Gamma^{*} / \Gamma\right|$, hence such $\Gamma$ is unimodular if and only if $\Gamma=\Gamma^{*}$. The classification of even unimodular lattices is known to some extent:

Theorem 2.0.2 Let $V$ denote a d-dimensional real vector space with scalar product $\langle\cdot, \cdot\rangle$ of signature $\left(\gamma_{+}, \gamma_{-}\right)$with $d=\gamma_{+}+\gamma_{-}>0$.

1. An even unimodular lattice $\Gamma \subset V$ exists if and only if $\gamma_{+}-\gamma_{-} \equiv 0 \bmod 8$.

2. If $\gamma_{+}>0$ and $\gamma_{-}>0$ and $\gamma_{+}-\gamma_{-} \equiv 0 \bmod 8$, then up to lattice isometries, there exists a unique even unimodular lattice $\Gamma \subset V$.

3. If $d=24$ and $\gamma_{-}=0$, then there are 24 distinct isometry classes of even unimodular lattices $\Gamma \subset V$. Each of these isometry classes is uniquely determined by the sublattice

$$
R:=\operatorname{span}_{\mathbb{Z}}\{\delta \in \Gamma \mid\langle\delta, \delta\rangle=2\}
$$

which has rank 0 or 24 and is isometric to the root lattice of some semi-simple complex Lie algebra.

Part 1. and 2. of the above theorem are known as Milnor's THEOREm (see [17] and [18, Ch. 5], [19, Ch. 2] for a proof), while the classification 3. of positive definite even unimodular lattices of rank 24 is due to NiEMEIER [20, and such lattices are named after him:

Definition 2.0.3 A NiEMEIER LATTICE is a positive definite even unimodular lattice $\Gamma$ of rank 24. Its ROOT SUBLATTICE (2.1) is the lattice $R \subset \Gamma$ that is generated by those elements of $\Gamma$ on which the quadratic form yields the value 2 . If $R$ is isometric to the root lattice of the semi-simple Lie algebra $\mathfrak{g}$, then we say that $\Gamma$ is a NIEMEIER LATTICE OF TYPE $\mathfrak{g}$.

In our applications, we often find ourselves in a situation where a primitive sublattice $\Lambda \subset \Gamma$ of an even unimodular lattice $\Gamma$ is well understood, and where we need to deduce properties of the lattice $\Gamma$ from those of $\Lambda$. In such situations, GLUING TECHNIQUES as well as CRITERIA FOR PRIMITIVITY OF SUBLATTICES that were developed by Nikulin in [11, 12, prove tremendously useful, see also [16. We recall these techniques in the next subsection.

\subsection{Even unimodular lattices from primitive sublattices}

If $\Gamma$ is an even unimodular lattice and $\Lambda \subset \Gamma$ is a primitive sublattice, then according to [11, Prop. 1.1] the discriminant forms $q_{\Lambda}$ and $q \mathcal{V}$ of $\Lambda$ and its orthogonal complement $\mathcal{V}:=\Lambda^{\perp} \cap \Gamma$ obey $q_{\Lambda}=-q \mathcal{V}$. Moreover,

Proposition 2.1.1 Let $\Gamma$ denote an even unimodular lattice and $\Lambda \subset \Gamma$ a non-degenerate primitive sublattice. Then the embedding $\Lambda \hookrightarrow \Gamma$ is uniquely determined by an isomorphism $\gamma: \Lambda^{*} / \Lambda \rightarrow \mathcal{V}^{*} / \mathcal{V}$, where $\mathcal{V} \cong \Lambda^{\perp} \cap \Gamma$ and the discriminant forms obey $q_{\Lambda}=-q_{\mathcal{V}} \circ \gamma$. Moreover,

$$
\Gamma \cong\left\{(\lambda, v) \in \Lambda^{*} \oplus \mathcal{V}^{*} \mid \gamma(\bar{\lambda})=\bar{v}\right\},
$$

where for $L$ a non-degenerate even lattice, $\bar{l}$ denotes the projection of $l \in L^{*}$ to $L^{*} / L$.

Note that (2.2) allows us to describe $\Gamma$ entirely by means of its sublattices $\Lambda$ and $\Lambda^{\perp} \cap \Gamma$ along with the isomorphism $\gamma$.

Example: Recall the HYPERBOLIC LATTICE, i.e. the even unimodular lattice of signature $(1,1)$ with quadratic form

$$
\left(\begin{array}{ll}
0 & 1 \\
1 & 0
\end{array}\right)
$$


with respect to generators $v_{0}, v$ over $\mathbb{Z}$. We generally denote the hyperbolic lattice by $U$. As a useful exercise, the reader should convince herself that the gluing procedure described above allows a reconstruction of $U$ from the two definite sublattices $A^{ \pm}$generated by $a_{ \pm}:=v_{0} \pm v$, respectively, with

$$
U=\left\{\frac{1}{2}\left(n_{+} a_{+}+n_{-} a_{-}\right) \mid n_{ \pm} \in \mathbb{Z}, n_{+}+n_{-} \in 2 \mathbb{Z}\right\}
$$

by (2.2).

To apply proposition 2.1.1 one needs to know that the lattice $\Lambda$ can be primitively embedded in some even, unimodular lattice. Nikulin has also developed powerful techniques which determine whether or not this is the case for abstract non-degenerate lattices $\Gamma$ and $\Lambda$. To recall this, we need some additional terminology:

Definition 2.1.2 Consider a finite abelian group $A$. The minimum number of generators of $A$ is called the LENGTH $\ell(A)$ OF $A$. If $A=A_{q}$ carries a quadratic form $q: A_{q} \rightarrow \mathbb{Q} / 2 \mathbb{Z}$, then for every prime $p$ let $A_{q_{p}}$ denote the SYLOW $p$-GROUP IN $A_{q}$, that is, $A_{q_{p}} \subset A_{q}$ is the maximal subgroup whose order is a power of $p$. Then by $q_{p}$ we denote the restriction of $q$ to $A_{q_{p}}$.

Note that for every finite abelian group $A$ and for every prime $p$, there exists a unique Sylow $p$-group in $A$. Moreover, if $A=A_{q}$ carries a quadratic form $q$ with values in $\mathbb{Q} / 2 \mathbb{Z}$, then $q$ decomposes into an orthogonal direct sum of all the $q_{p}$ with prime $p$. As a special case of [12, Thm. 1.12.2] used in precisely this form in Kondo's proof of [10, Lemma 5], we now have:

Theorem 2.1.3 Let $\Lambda$ denote a non-degenerate even lattice of signature $\left(l_{+}, l_{-}\right)$and $A_{q}=$ $\Lambda^{*} / \Lambda$ its discriminant group, equipped with the induced quadratic form $q$ with values in $\mathbb{Q} / 2 \mathbb{Z}$. Furthermore assume that all the following conditions hold for the integers $\gamma_{+}, \gamma_{-}$with $\gamma_{+} \equiv \gamma_{-}$ $\bmod 8$ :

1. $\gamma_{+} \geq l_{+}$and $\gamma_{-} \geq l_{-}$,

2. $\ell\left(A_{q}\right) \leq \gamma_{+}+\gamma_{-}-l_{+}-l_{-}$,

3. $i . q_{2}=\left(\frac{1}{2}\right) \oplus q_{2}^{\prime}$, where $\left(\frac{1}{2}\right)$ is the quadratic form on the discriminant of a root lattice $\mathbb{Z}(2)$ of type $A_{1}$ and $q_{2}^{\prime}$ is an arbitrary quadratic form with values in $\mathbb{Q} / 2 \mathbb{Z}$ or ii. $\ell\left(A_{q_{2}}\right)<\gamma_{+}+\gamma_{-}-l_{+}-l_{-}$,

4. for every prime $p \neq 2, \ell\left(A_{q_{p}}\right)<\gamma_{+}+\gamma_{-}-l_{+}-l_{-}$.

Then $\Lambda$ can be primitively embedded in some even unimodular lattice $\Gamma$ of signature $\left(\gamma_{+}, \gamma_{-}\right)$.

\subsection{Example: The K3-lattice for Kummer surfaces}

A classical application of the gluing technique summarized in proposition 2.1.1 is the description of the integral homology of a Kummer surface in terms of the integral homology of its underlying torus and the contributions from the blow-up of singularities. We review this construction in the present subsection, following [21, 22]. First recall the definition of K3 surfaces:

Definition 2.2.1 A K3 SURFACE is a compact complex surface with trivial canonical bundle and vanishing first Betti number. 
By a seminal result of Kodaira's [23, Thm. 19], as real four-manifolds all K3 surfaces are diffeomorphic. The integral homology of a K3 surface $X$ has the following properties (see e.g. [17, 24]): First, $H_{2}(X, \mathbb{Z})$ has no torsion, and equipped with the intersection form it is an even unimodular lattice of signature $(3,19)$. By theorem 2.0 .2 this means that $H_{2}(X, \mathbb{Z})$ is uniquely determined up to isometry. In fact, $H_{2}(X, \mathbb{Z}) \cong U^{3} \oplus E_{8}^{2}(-1)$, where $U$ is the hyperbolic lattice with quadratic form (2.3) and $E_{8}$ is isometric to the root lattice of the Lie algebra $e_{8}$. The lattice $U^{3} \oplus E_{8}^{2}(-1)$ is often called the K3-LATTICE. Furthermore, $H_{*}(X, \mathbb{Z})=H_{0}(X, \mathbb{Z}) \oplus H_{2}(X, \mathbb{Z}) \oplus H_{4}(X, \mathbb{Z})$ is an even unimodular lattice of signature $(4,20)$ and thus $H_{*}(X, \mathbb{Z}) \cong U^{4} \oplus E_{8}^{2}(-1)$ by theorem 2.0 .2 and the above. A choice of isometries $H_{2}(X, \mathbb{Z}) \cong U^{3} \oplus E_{8}^{2}(-1), H_{*}(X, \mathbb{Z}) \cong U^{4} \oplus E_{8}^{2}(-1)$ is called a MARKING of our K3 surface $X$. Such a marking is specified by fixing a standard basis of $H_{2}(X, \mathbb{Z}), H_{*}(X, \mathbb{Z})$, which does not necessarily need to exhibit the structure of $U^{3} \oplus E_{8}^{2}(-1), U^{4} \oplus E_{8}^{2}(-1)$ in the first instance.

Let us now recall the KUMMER CONSTRUCTION, which yields a K3 surface by a $\mathbb{Z}_{2}$-orbifold construction from every complex torus $T$ of dimension 2. Let $T=T(\Lambda)=\mathbb{C}^{2} / \Lambda$, with $\Lambda \subset \mathbb{C}^{2}$ a lattice of rank 4 over $\mathbb{Z}$, and with generators $\overrightarrow{\lambda_{i}}, i=1, \ldots, 4$. The group $\mathbb{Z}_{2}$ acts naturally on $\mathbb{C}^{2}$ by $\left(z_{1}, z_{2}\right) \mapsto\left(-z_{1},-z_{2}\right)$ and thereby on $T(\Lambda)$. Using Euclidean coordinates $\vec{x}=\left(x_{1}, x_{2}, x_{3}, x_{4}\right)$, where $z_{1}=x_{1}+i x_{2}$ and $z_{2}=x_{3}+i x_{4}$, points on the quotient $T(\Lambda) / \mathbb{Z}_{2}$ are identified according to

$$
\begin{aligned}
& \vec{x} \sim \vec{x}+\sum_{i=1}^{4} n_{i} \vec{\lambda}_{i}, \quad n_{i} \in \mathbb{Z}, \\
& \vec{x} \sim-\vec{x} .
\end{aligned}
$$

Hence $T(\Lambda) / \mathbb{Z}_{2}$ has 16 singularities of type $A_{1}$, located at the fixed points of the $\mathbb{Z}_{2}$-action. These fixed points are conveniently labelled by the HYPERCUBE $\mathbb{F}_{2}^{4} \cong \frac{1}{2} \Lambda / \Lambda$, where $\mathbb{F}_{2}=\{0,1\}$ is the finite field with two elements, as

$$
\vec{F}_{\vec{a}}:=\left[\frac{1}{2} \sum_{i=1}^{4} a_{i} \overrightarrow{\lambda_{i}}\right] \in T(\Lambda) / \mathbb{Z}_{2}, \quad \vec{a}=\left(a_{1}, a_{2}, a_{3}, a_{4}\right) \in \mathbb{F}_{2}^{4} .
$$

It is known that the complex surface obtained by minimally resolving $T(\Lambda) / \mathbb{Z}_{2}$ is a $\mathrm{K} 3$ surface (see e.g. [22]):

Definition 2.2.2 Let $T=T(\Lambda)$ denote a complex torus of dimension 2 with underlying lattice $\Lambda \subset \mathbb{C}^{2}$. The Kummer SURFACE WITH UNDERLYING TORUs $T$ is the complex surface $X$ obtained by minimally resolving each of the singularities $\vec{F}_{\vec{a}}, \vec{a} \in \mathbb{F}_{2}^{4} \cong \frac{1}{2} \Lambda / \Lambda$, of $T / \mathbb{Z}_{2}$ given in (2.4). The blow-up of $\vec{F}_{\vec{a}}$ yields an exceptional divisor whose class in $H_{2}(X, \mathbb{Z})$ we denote by $E_{\vec{a}}$. The natural rational map of degree 2 from $T$ to $X$, which is defined outside the fixed points of $\mathbb{Z}_{2}$ on $T$, is denoted $\pi: T \rightarrow X$, and the linear map it induces on homology is denoted $\pi_{*}: H_{2}(T, \mathbb{Z}) \rightarrow H_{2}(X, \mathbb{Z}), \pi_{*}: H_{2}(T, \mathbb{R}) \rightarrow H_{2}(X, \mathbb{R})$, respectively.

Note that by definition, the Kummer surface $X$ with underlying torus $T(\Lambda)=\mathbb{C}^{2} / \Lambda$ carries the complex structure which is induced from the universal cover $\mathbb{C}^{2}$ of $T(\Lambda)$. Moreover, the integral classes $E_{\vec{a}} \in H_{2}(X, \mathbb{Z})$ with $\vec{a} \in \mathbb{F}_{2}^{4}$ arise from blowing up singularities of type $A_{1}$ and thus represent rational two-cycles on $X$. Hence by construction, they generate a sublattice of $H_{2}(X, \mathbb{Z})$ of type $A_{1}^{16}(-1)$, i.e. a sublattice of rank 16 with quadratic form $\operatorname{diag}(-2, \ldots,-2)$. This lattice, however, is not primitively embedded in $H_{2}(X, \mathbb{Z})$. Instead, by [21, 22] we have

Proposition 2.2.3 Let $X$ denote a Kummer surface with underlying torus $T$ as in definition 2.2.2. Furthermore, let $\Pi$ denote the smallest primitive sublattice of $H_{2}(X, \mathbb{Z})$ containing all the $E_{\vec{a}}, \vec{a} \in \mathbb{F}_{2}^{4}$, and let $K:=\pi_{*}\left(H_{2}(T, \mathbb{Z})\right)$. Then the following holds: 


\section{The lattice $\Pi$ is the Kummer LATTice}

$$
\Pi=\operatorname{span}_{\mathbb{Z}}\left\{E_{\vec{a}} \text { with } \vec{a} \in \mathbb{F}_{2}^{4} ; \frac{1}{2} \sum_{\vec{a} \in H} E_{\vec{a}} \text { with } H \subset \mathbb{F}_{2}^{4} \text { an affine hyperplane }\right\} .
$$

The embedding of $\Pi$ in $H_{2}(X, \mathbb{Z})$ is unique up to automorphisms of $H_{2}(X, \mathbb{Z})$.

2. The lattice $K$ is a primitive sublattice of $H_{2}(X, \mathbb{Z})$ with $K \cong U^{3}(2)$ and $K=\Pi^{\perp} \cap$ $\mathrm{H}_{2}(X, \mathbb{Z})$.

Note that $K \cong U^{3}(2)$ follows by construction, since $H_{2}(T, \mathbb{Z}) \cong U^{3}$ and $\pi$ has degree 2 . Moreover, each element of $K$ represents the image of a two-cycle on the torus which is in general position and thus does not contain any fixed points of the $\mathbb{Z}_{2}$-action, hence $K \perp \Pi$. Then $K=\Pi^{\perp} \cap H_{2}(X, \mathbb{Z})$ since $K$ is a primitive sublattice of $\Pi^{\perp} \cap H_{2}(X, \mathbb{Z})$ of same rank $6=22-16$.

For the remainder of this work, we continue to use the notations introduced in definition 2.2.2 and proposition 2.2 .3 above. According to this proposition, $K$ and $\Pi$ are orthogonal complements of one another in the even unimodular lattice $H_{2}(X, \mathbb{Z})$. By the gluing construction of proposition 2.1.1, $H_{2}(X, \mathbb{Z})$ can therefore be reconstructed from its sublattices $K$ and $\Pi$.

Indeed, one first checks $K^{*} / K \cong \Pi^{*} / \Pi \cong\left(\mathbb{Z}_{2}\right)^{6}$ : With

$$
\lambda_{i j}:=\lambda_{i} \vee \lambda_{j} \in H_{2}(T, \mathbb{Z}) \text { for } i, j \in\{1,2,3,4\},
$$

standard generators of $K^{*} / K$ and the discriminant form with respect to these generators are given by

$$
\overline{\frac{1}{2} \pi_{*} \lambda_{i j}}, i j=12,34,13,24,14,23, \quad q_{K}=\left(\begin{array}{cc}
0 & \frac{1}{2} \\
\frac{1}{2} & 0
\end{array}\right)^{3} .
$$

Analogously, with

$$
P_{i j}:=\left\{\vec{a}=\left(a_{1}, a_{2}, a_{3}, a_{4}\right) \in \mathbb{F}_{2}^{4} \mid a_{k}=0 \forall k \neq i, j\right\} \text { for } i, j \in\{1,2,3,4\},
$$

standard generators of $\Pi^{*} / \Pi$ and the discriminant form with respect to these generators are given by

$$
\overline{\frac{1}{2} \sum_{\vec{a} \in P_{i j}} E_{\vec{a}}}, i j=12,34,13,24,14,23, \quad q_{\Pi}=-\left(\begin{array}{cc}
0 & \frac{1}{2} \\
\frac{1}{2} & 0
\end{array}\right)^{3} .
$$

The bilinear forms associated with $q_{K}, q_{\Pi}$ take values in $\mathbb{Q} / \mathbb{Z}$ and thus $q_{K}=-q_{K}, q_{\Pi}=-q_{\Pi}$. Hence we obtain a natural isomorphism $\gamma$ between the two discriminant groups which obeys $q_{K}=-q_{\Pi} \circ \gamma:$

$$
\gamma: K^{*} / K \longrightarrow \Pi^{*} / \Pi, \quad \gamma\left(\overline{\frac{1}{2} \pi_{*} \lambda_{i j}}\right):=\overline{\frac{1}{2} \sum_{\vec{a} \in P_{i j}} E_{\vec{a}}} .
$$

Now proposition 2.1 .1 implies that the K3-lattice $H_{2}(X, \mathbb{Z})$ is generated by the $\pi_{*} \lambda_{i j} \in$ $\pi_{*}\left(H_{2}(T, \mathbb{Z})\right)$, the elements of the Kummer lattice $\Pi$, and two-cycles of type $\frac{1}{2} \pi_{*} \lambda_{i j}+\frac{1}{2} \sum_{\vec{a} \in P_{i j}} E_{\vec{a}} \in$ $K^{*} \oplus \Pi^{*}$. In this case, the gluing procedure can be visualised geometrically as follows: Consider a real 2-dimensional subspace of $\mathbb{C}^{2}$ which on the torus $T$ yields a $\mathbb{Z}_{2}$-invariant submanifold $\kappa$ containing the four fixed points labelled by a plane $P \subset \mathbb{F}_{2}^{4}$. Then $\kappa \rightarrow \kappa / \mathbb{Z}_{2}$ is a $2: 1$ cover of an $\mathbb{S}^{2}$ with branch points $\vec{F}_{\vec{a}}, \vec{a} \in P$, which under blow-up are replaced by the corresponding exceptional divisors representing the $E_{\vec{a}} \in H_{2}(X, \mathbb{Z})$. Hence $\left(\kappa \backslash\left\{\vec{F}_{\vec{a}} \mid \vec{a} \in P\right\}\right)$ is a $2: 1$ unbranched covering of a two-cycle on the Kummer surface $X$ representing $\pi_{*}[\kappa]-\sum_{\vec{a} \in P} E_{\vec{a}} \in$

\footnotetext{
${ }^{3}$ The generators $\vec{\lambda}_{i}, i=1, \ldots, 4$, of the lattice $\Lambda$ are naturally identified with generators $\lambda_{i}, i=1, \ldots, 4$, of $H_{1}(T, \mathbb{Z})$, such that $H_{2}(T, \mathbb{Z})$ is generated by the $\lambda_{i} \vee \lambda_{j}$.
} 
$H_{2}(X, \mathbb{Z})$. In other words, $\frac{1}{2} \pi_{*}[\kappa] \mp \frac{1}{2} \sum_{\vec{a} \in P} E_{\vec{a}} \in H_{2}(X, \mathbb{Z})$. Indeed, note that for $P$ as above and $P^{\prime} \subset \mathbb{F}_{2}^{4}$ a plane parallel to $P, \frac{1}{2} \sum_{\vec{a} \in P} E_{\vec{a}} \mp \frac{1}{2} \sum_{\vec{a} \in P^{\prime}} E_{\vec{a}} \in \Pi$ according to (2.5).

For later use, instead of restricting our attention to the K3-lattice $H_{2}(X, \mathbb{Z})$ of a Kummer surface $X$, we need to work on the full integral homology $H_{*}(X, \mathbb{Z})=H_{0}(X, \mathbb{Z}) \oplus H_{2}(X, \mathbb{Z}) \oplus$ $H_{4}(X, \mathbb{Z})$. Since $H_{0}(X, \mathbb{Z}) \oplus H_{4}(X, \mathbb{Z}) \cong U$ is an even unimodular lattice, we can use the gluing prescription (2.8) either replacing $K$ by $K \oplus U$ with $(K \oplus U)^{*} /(K \oplus U) \cong K^{*} / K$, or replacing $\Pi$ by $\Pi \oplus U$ with $(\Pi \oplus U)^{*} /(\Pi \oplus U) \cong \Pi^{*} / \Pi$. However, yet another option will turn out to be even more useful: We combine the gluing prescription (2.8) with the exercise posed in section 2.1 to obtain, as another application of proposition 2.1.1,

Proposition 2.2.4 Consider a Kummer surface $X$ with underlying torus $T$ and notations as in definition 2.2 .2 and proposition 2.2 .3 . Furthermore, let $v_{0} \in H_{0}(X, \mathbb{Z})$ and $v \in H_{4}(X, \mathbb{Z})$ denote generators of $H_{0}(X, \mathbb{Z}) \oplus H_{4}(X, \mathbb{Z}) \cong U$ such that the quadratic form with respect to $v_{0}, v$ is (2.3). Let

$$
\mathcal{K}:=K \oplus \operatorname{span}_{\mathbb{Z}}\left\{v_{0}+v\right\}, \quad \mathcal{P}:=\Pi \oplus \operatorname{span}_{\mathbb{Z}}\left\{v_{0}-v\right\} .
$$

Then $\mathcal{K}^{*} / \mathcal{K} \cong \mathcal{P}^{*} / \mathcal{P} \cong\left(\mathbb{Z}_{2}\right)^{7}$ under an isomorphism g with

$$
\begin{aligned}
g: \quad & \mathcal{K}^{*} / \mathcal{K} \longrightarrow \mathcal{P}^{*} / \mathcal{P}, \quad g(\bar{\kappa}):=\gamma(\bar{\kappa}) \forall \kappa \in K^{*}, \quad g\left(\overline{\frac{1}{2}\left(v_{0}+v\right)}\right):=\overline{\frac{1}{2}\left(v_{0}-v\right)} ; \\
& H_{*}(X, \mathbb{Z}) \cong\left\{(\kappa, \pi) \in \mathcal{K}^{*} \oplus \mathcal{P}^{*} \mid g(\bar{\kappa})=\bar{\pi}\right\} .
\end{aligned}
$$

By the above, the Kummer construction yields a natural marking $H_{2}(X, \mathbb{Z}) \cong U^{3} \oplus E_{8}^{2}(-1)$, $H_{*}(X, \mathbb{Z}) \cong U^{4} \oplus E_{8}^{2}(-1)$. Indeed, the $\lambda_{i j}, i, j \in\{1, \ldots, 4\}, i<j$, form a basis of $H_{2}(T, \mathbb{Z})$, where each of the pairs $\left\{\lambda_{12}, \lambda_{34}\right\},\left\{\lambda_{13}, \lambda_{24}\right\},\left\{\lambda_{14}, \lambda_{23}\right\}$ generates a sublattice which is isometric to the hyperbolic lattice $U$. Furthermore, (2.5) gives an abstract construction of the Kummer lattice $\Pi$ in terms of the roots $E_{\vec{a}}, \vec{a} \in \mathbb{F}_{2}^{4}$, with $\left\langle E_{\vec{a}}, E_{\vec{b}}\right\rangle=-2 \delta_{\vec{a}, \vec{b}}$. Then the above gluing prescription of $H_{2}(X, \mathbb{Z})$ from the lattices $\pi_{*} H_{2}(T, \mathbb{Z})$ and $\Pi$ specifies an isometry between $H_{2}(X, \mathbb{Z})$ for $X=\widetilde{T / \mathbb{Z}_{2}}$ with a standard even, unimodular lattice of signature $(3,19)$ (though not written in the form $U^{3} \oplus E_{8}^{2}(-1)$ ). Denoting generators of $H_{0}(X, \mathbb{Z})$ and $H_{4}(X, \mathbb{Z})$ by $v_{0}, v$, respectively, where $\left\langle v_{0}, v\right\rangle=1$ as in proposition 2.2.4, we obtain $H_{*}(X, \mathbb{Z}) \cong U^{4} \oplus E_{8}^{2}(-1)$.

In all examples studied below, we use this fixed marking, as it is natural for all Kummer surfaces. Note that the marking allows us to smoothly vary the generators $\vec{\lambda}_{1}, \ldots, \vec{\lambda}_{4}$ of the defining lattice $\Lambda \subset \mathbb{C}^{2}$ of $T=T(\Lambda)$ for $X=T \widetilde{T(\Lambda) / \mathbb{Z}_{2}}$; the marking is thus compatible with the deformation of any Kummer surface into any other one.

\subsection{Example: The Niemeier lattice of type $A_{1}^{24}$}

A second example for the application of Nikulin's gluing techniques from proposition 2.1.1, which we find extremely useful, involves the Niemeier lattice $N$ of type $A_{1}^{24}$ (see theorem 2.0.2 and definition 2.0.3). In other words, $N$ is the Niemeier lattice with root sublattice $R \subset N$ of rank 24 , where $R$ has quadratic form $\operatorname{diag}(2, \ldots, 2)$. Since $N$ is unimodular, we have

$$
R \subset N \subset R^{*},
$$

where $R^{*}=\frac{1}{2} R$ and thus $R^{*} / R \cong \mathbb{F}_{2}^{24}$. Hence $N / R$ can be viewed as a subspace of $\mathbb{F}_{2}^{24}$, and in fact $N / R \cong \mathcal{G}_{24}$, the EXTENDED BINARY GolaY CODE [25, Ch. 16, 18]. Up to isometry, the extended binary Golay code is uniquely determined by the fact that it is a 12-dimensional subspace of $\mathbb{F}_{2}^{24}$ over $\mathbb{F}_{2}$ such that every $v \in \mathcal{G}_{24}$ has Weight 4 zero, 8 (OCTAD), 12 (DODECAD),

\footnotetext{
${ }^{4}$ that is, the number of non-zero entries
} 
16 (COMPLEMENT OCTAD), or 24. For further details concerning the extended binary Golay code, which for brevity we simply call the GOLAY CODE from now on, see appendix A.

In terms of the Golay code $\mathcal{G}_{24} \subset \mathbb{F}_{2}^{24}$, the Niemeier lattice $N$ can be constructed from its root sublattice $R$ as a sublattice of $R^{*}=\frac{1}{2} R$ :

Proposition 2.3.1 Consider the Niemeier lattice of type $A_{1}^{24}$, which for the remainder of this work is denoted by $N$, and its root sublattice $R$. Then

$$
N=\left\{v \in R^{*} \mid \bar{v} \in \mathcal{G}_{24}\right\},
$$

where $\bar{v}$ denotes the projection of $v \in R^{*}$ to $\mathbb{F}_{2}^{24} \cong R^{*} / R$.

As stated in theorem 2.0.2, every Niemeier lattice $\tilde{N}$ is uniquely determined by its root sublattice $\widetilde{R}$ up to isometry. In fact, if $\widetilde{R}$ has rank 24 , then $\widetilde{N}$ can always be constructed from $\widetilde{R}$ analogously to proposition 2.3.1. In this paper, the Niemeier lattice $N$ of type $A_{1}^{24}$ plays a special role, though, since the MATHIEU GROUP $M_{24}$ acts so naturally on it 5 . Namely, $M_{24}$ is the automorphism group of the Golay code [27, 28, 29], and thus we determine the automorphisms of $N$ in accord with [25, Ch. 4.3, 16.1]:

Proposition 2.3.2 Consider the Niemeier lattice $N$ of type $A_{1}^{24}$ with root sublattice $R$ generated by the roots $f_{1}, \ldots, f_{24}$. The automorphism group of $N$ is

$$
\operatorname{Aut}(N)=\left(\mathbb{Z}_{2}\right)^{24} \rtimes M_{24},
$$

where the action of the $n^{\text {th }}$ factor of $\left(\mathbb{Z}_{2}\right)^{24}$ is induced by $f_{n} \mapsto-f_{n}$, while the MATHIEU GROUP $M_{24}$ is viewed as a subgroup of the permutation group $S_{24}$ on 24 elements whose action on $N$ is induced by permuting the roots $f_{1}, \ldots, f_{24}$.

Proof: Since every non-trivial codeword in the Golay code has at least weight 8, from proposition 2.3 .1 we see that the set $\Delta$ of roots in $N$ is

$$
\Delta=\{\delta \in N \mid\langle\delta, \delta\rangle=2\}=\left\{ \pm f_{1}, \ldots, \pm f_{24}\right\} .
$$

Hence every lattice isometry $\gamma \in \operatorname{Aut}(N)$ acts as a permutation on $\Delta$. Proposition 2.3.1 implies $N \subset \frac{1}{2} R$ and thus that $\gamma$ is uniquely determined by its action on $\Delta$. Thus $\gamma=\iota \circ \alpha$ with $\iota \in\left(\mathbb{Z}_{2}\right)^{24} \subset \operatorname{Aut}(N)$ induced by a composition of involutions $f_{n} \mapsto-f_{n}$, and with $\alpha \in \operatorname{Aut}(N)$ induced by a permutation of $f_{1}, \ldots, f_{24}$. Now proposition 2.3.1 implies that $\alpha$ induces an action on $\mathbb{F}_{2}^{24} \cong R^{*} / R$ by permuting the binary coordinates of $\mathbb{F}_{2}^{24}$, which must leave the Golay code $\mathcal{G}_{24} \subset \mathbb{F}_{2}^{24}$ invariant. In other words, $\alpha \in M_{24}$. Vice versa, every involution $f_{n} \mapsto-f_{n}$ and every permutation of the binary coordinates of $\mathbb{F}_{2}^{24}$ which preserves the Golay code induces an automorphism of $N$. From this the claim follows.

The above proposition allows us to view the Niemeier lattice $N$ as a device which yields a geometric interpretation of the Mathieu group $M_{24}$. On the other hand, this lattice turns out to share a number of properties with the integral homology of Kummer surfaces that we discussed in section 2.2. First we observe

Proposition 2.3.3 The Niemeier lattice $N$ of type $A_{1}^{24}$ possesses a primitive sublattice $\widetilde{\Pi} \subset$ $N$ which up to a total reversal of signature is isometric to the Kummer lattice (2.5).

No other Niemeier lattice possesses such a sublattice.

\footnotetext{
${ }^{5}$ By giving an extensive list of examples, in 26] Nikulin emphasizes that all 24 Niemeier lattices are important for the study of K3 surfaces; while this may be true, our objective is the clarification of the role of the Mathieu group $M_{24}$, justifying our preference for the lattice $N$.
} 
Proof: Given the Kummer lattice $\Pi$ of (2.5), consider the lattice $\widetilde{\Pi}:=\Pi(-1)$. We first show that $\widetilde{\Pi}$ can be primitively embedded in some Niemeier lattice $\widetilde{N}$ by applying theorem 2.1.3 to the lattice $\Lambda=\widetilde{\Pi}$.

Recall from definition 2.0.3 that a Niemeier lattice is an even unimodular lattice of signature $\left(\gamma_{+}, \gamma_{-}\right)=(24,0)$. Furthermore, by proposition 2.2.3 and the explanations following it, the lattice $\widetilde{\Pi}$ is a non-degenerate even lattice with signature $\left(l_{+}, l_{-}\right)=(16,0)$ and discriminant group $A_{q}=\widetilde{\Pi}^{*} / \widetilde{\Pi} \cong\left(\mathbb{Z}_{2}\right)^{6}$. In particular, the length of this group is $\ell\left(A_{q}\right)=6$, and for the reductions modulo primes we find $\ell\left(A_{q_{2}}\right)=6$ and $\ell\left(A_{q_{p}}\right)=1$ for all primes $p \neq 2$. Since $\gamma_{+}+\gamma_{-}-l_{+}-l_{-}=\gamma_{+}-l_{+}=8>6>1$, the conditions 1., 2., 3.ii. and 4. of theorem 2.1.3 hold, and thus $\widetilde{\Pi}$ can be primitively embedded in some Niemeier lattice $\widetilde{N}$.

For every Niemeier lattice $\widetilde{N}$, the root lattice $\widetilde{R}$ is known (see e.g. [25, Ch. 16.1]), and one checks that the Niemeier lattice $N$ of type $A_{1}^{24}$ is the only one containing 16 pairwise perpendicular roots that generate a sublattice $R$ of type $A_{1}^{16}$, such that $(R \otimes \mathbb{Q}) \cap N$ contains no further roots, as $\widetilde{\Pi}$ does. From this the claim follows.

In 26, Case 23], Nikulin remarks that $N$ is the only Niemeier lattice which can contain a primitive sublattice that up to a total signature reversal is isomorphic to the Kummer lattice 6 For our investigations, we need an EXPLICIT REALISATION OF A PRIMITIVE SUBLATTICE $\widetilde{\Pi}$ as in proposition 2.3.3. Our construction of the lattice $\widetilde{\Pi}$ and its orthogonal complement $\widetilde{K}$ in $N$ depends on the choice of an arbitrary SPECIAL OCTAD in the Golay code, i.e. a vector of weight 8 in $\mathcal{G}_{24}$. For historical reasons, our choice of special octad is the codeword $o_{9}:=(0,0,1,0,1,1,0,0,1,0,0,0,0,0,1,0,0,0,1,0,0,0,1,1) \in \mathcal{G}_{24}$ whose non-zero entries are the binary coordinates with labels $\{3,5,6,9,15,19,23,24\} \subset \mathcal{I}$ with $\mathcal{I}:=\{1, \ldots, 24\}$. For ease of notation, we regularly denote a codeword $v \in \mathcal{G}_{24} \subset \mathbb{F}_{2}^{24}$ of the Golay code by listing the set $A_{v} \subset \mathcal{I}$ of coordinate labels with non-zero entries. With this notation, calculating the sum of codewords $v, w \in \mathcal{G}_{24} \subset \mathbb{F}_{2}^{24}$ amounts to taking the SYMMETRIC DIFFERENCE of sets $A_{v}+A_{w}=\left(A_{v} \backslash A_{w}\right) \cup\left(A_{w} \backslash A_{v}\right)$. Our special octad $o_{9}$ is thus described by

$$
\begin{aligned}
\mathcal{O}_{9} & :=\{3,5,6,9,15,19,23,24\} \\
o_{9} & =(0,0,1,0,1,1,0,0,1,0,0,0,0,0,1,0,0,0,1,0,0,0,1,1) \in \mathcal{G}_{24} \subset \mathbb{F}_{2}^{24} .
\end{aligned}
$$

This is the octad corresponding to the standard MOG configuration described in appendix $\mathrm{A}$, where the two first columns have entries 1 , and the others are 0 . We now claim the following

Proposition 2.3.4 Consider the Niemeier lattice $N$ of type $A_{1}^{24}$ with root sublattice $R$ generated by roots $f_{1}, \ldots, f_{24}$. With our choice (2.10) of a special octad $\mathcal{O}_{9}$ let

$$
\widetilde{K}:=\left\{\nu \in N \mid \forall n \notin \mathcal{O}_{9}:\left\langle\nu, f_{n}\right\rangle=0\right\}, \quad \widetilde{\Pi}:=\left\{\nu \in N \mid \forall n \in \mathcal{O}_{9}:\left\langle\nu, f_{n}\right\rangle=0\right\} .
$$

Then $\widetilde{K}$ and $\widetilde{\Pi}$ are primitive sublattices of $N$ which are orthogonal complements of one another. Moreover, $\widetilde{\Pi}(-1)$ is isometric to the Kummer lattice $\Pi$ as in (2.5).

Proof: That $\widetilde{K}$ and $\widetilde{\Pi}$ are primitive sublattices of $N$ follows immediately from (2.11). For $n \in \mathcal{I}$, by construction, $f_{n} \in \widetilde{K}$ if and only if $n \in \mathcal{O}_{9}$, while $f_{n} \in \widetilde{\Pi}$ if and only if $n \notin \mathcal{O}_{9}$. Thus $\widetilde{K}$ has rank at least 8 and $\widetilde{\Pi}$ has rank at least 16 with $\widetilde{K}^{\perp} \cap N \subset \widetilde{\Pi}, \widetilde{\Pi}^{\perp} \cap N \subset \widetilde{K}$. Thus $8+16=24=\operatorname{rk}(N)$ implies that these lattices are orthogonal complements of one another. To prove that $\widetilde{\Pi}(-1)$ is isometric to the Kummer lattice $\Pi$ given in (2.5), first observe that there is a 5 -dimensional subspace of the Golay code $\mathcal{G}_{24}$, defined as the space of all those

\footnotetext{
${ }^{6}$ Nikulin however fails to mention that the existence of a primitive sublattice $\widetilde{\Pi}$ in $N$ as in proposition 2.3 .3 was first observed and proved in our first installment arXiv:1008.0954 of this work. In fact he fails to observe that existence needs to be proved, whatsoever.
} 
codewords which have no intersection with the octad $\mathcal{O}_{9}$. A basis of this space is

$$
\begin{aligned}
& \mathcal{H}_{1}:=\{1,2,4,12,13,14,17,18\}, \\
& \mathcal{H}_{2}:=\{1,2,8,11,14,16,17,22\}, \\
& \mathcal{H}_{3}:=\{1,8,10,11,13,17,18,21\}, \\
& \mathcal{H}_{4}:=\{1,4,11,13,14,16,20,21\}, \\
& \mathcal{H}_{5}:=\{2,7,8,10,12,17,18,22\} .
\end{aligned}
$$

Hence we have

$$
\widetilde{\Pi}=\operatorname{span}_{\mathbb{Z}}\left\{f_{n}, n \notin \mathcal{O}_{9} ; \frac{1}{2} \sum_{n \in \mathcal{H}_{i}} f_{n}, i=1, \ldots, 5\right\} .
$$

Now consider the map $I: \mathcal{I} \backslash \mathcal{O}_{9} \longrightarrow \mathbb{F}_{2}^{4}$ with

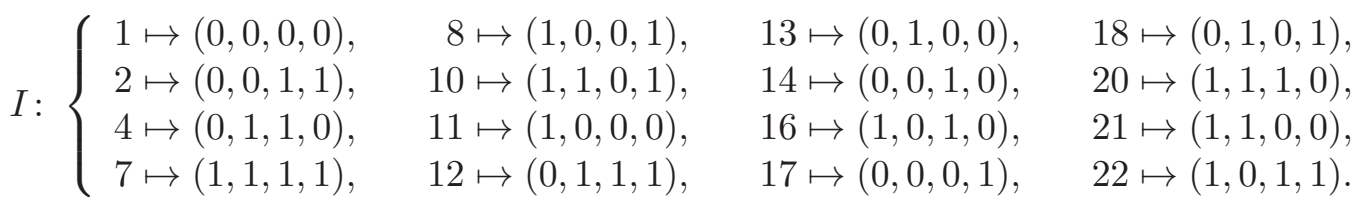

Under this map, the elements of $\mathcal{H}_{i}$ with $i=1, \ldots, 4$ correspond to the hypercube points $\vec{a}=\left(a_{1}, a_{2}, a_{3}, a_{4}\right) \in \mathbb{F}_{2}^{4}$ with $a_{i}=0$, while the hypercube points corresponding to elements of $\mathcal{H}_{5}$ are obtained from those corresponding to $\mathcal{H}_{4}$ by a shift by $(1,1,1,1) \in \mathbb{F}_{2}^{4}$. In other words, in terms of the hypercube labels, each $\mathcal{H}_{i}$ contains the labels corresponding to an affine hyperplane $H_{i} \subset \mathbb{F}_{2}^{4}$, such that every hyperplane in $\mathbb{F}_{2}^{4}$ can be obtained from $H_{1}, \ldots, H_{5}$ by means of symmetric differences. Hence (2.5) and (2.13) show that the map $f_{n} \mapsto E_{I(n)}$ for $n \notin \mathcal{O}_{9}$ induces an isometry of lattices $\widetilde{\Pi}(-1) \longrightarrow \Pi$.

To our knowledge, the relation of the Golay code to the Kummer lattice found in proposition 2.3.4 is a new observation. It is certainly crucial for our analysis below. Our map $I$ in (2.14) induces the structure of a 4-dimensional vector space over $\mathbb{F}_{2}$ on the 16 labels of the Golay code in $\mathcal{I} \backslash \mathcal{O}_{9}$. This linear structure is known to group theorist:7: In [29, Thm. 2.10], Conway proves the previously known fact (see e.g. [30]) that the general linear group $L_{4}(2)=\operatorname{PSL}\left(4, \mathbb{F}_{2}\right)=$ $\mathrm{GL}\left(4, \mathbb{F}_{2}\right)$ of $\mathbb{F}_{2}^{4}$ is isomorphic to the permutation group $A_{8}$ of even permutations on 8 elements. In our notations, the crucial step in the proof identifies the action of the stabilizer subgroup of $\mathcal{O}_{9} \cup\{7\}$ in $M_{24}$ with the action of a subgroup of $L_{4}(2)$ on the 4-dimensional subspace of $\mathcal{G}_{24}$ generated by the octads $\mathcal{H}_{1}, \ldots, \mathcal{H}_{4}$ as in (2.12). By our construction, this vector space is the dual of our hypercube $\mathbb{F}_{2}^{4}$ built on $\mathcal{I} \backslash \mathcal{O}_{9}$. The vector space structure of the latter, to our knowledge, is first mentioned by Curtis in [31. Hence altogether our proposition 2.3.4 gives a novel geometric meaning in terms of Kummer geometry to the known vector space structure on $\mathcal{I} \backslash \mathcal{O}_{9}$.

Note that both lattices $\widetilde{K}$ and $\widetilde{\Pi}$ in proposition 2.3.4 are contained in the $\mathbb{Q}$-span of their root sublattices. The gluing techniques of proposition 2.1.1 apply and allow us to reconstruct the Niemeier lattice $N$ from these lattices. Indeed, first note that $\widetilde{K}^{*} / \widetilde{K} \cong \widetilde{\Pi}^{*} / \widetilde{\Pi} \cong\left(\mathbb{Z}_{2}\right)^{6}$ with associated discriminant forms obeying $q_{\widetilde{K}}=-q_{\widetilde{\Pi}}$. Namely, as representatives $q_{i j} \in K^{*}$ of a minimal set of generators of $\widetilde{K}^{*} / \widetilde{K}$ we identify, for example,

$$
\begin{aligned}
q_{12}:=\frac{1}{2}\left(f_{3}+f_{6}-f_{15}-f_{19}\right), & q_{34}:=\frac{1}{2}\left(f_{6}+f_{9}-f_{15}-f_{19}\right), \\
q_{13}:=\frac{1}{2}\left(-f_{6}+f_{15}-f_{23}+f_{24}\right), & q_{24}:=\frac{1}{2}\left(-f_{15}+f_{19}+f_{23}-f_{24}\right), \\
q_{14}:=\frac{1}{2}\left(f_{3}-f_{9}-f_{15}+f_{24}\right), & q_{23}:=\frac{1}{2}\left(f_{3}-f_{9}-f_{15}+f_{23}\right),
\end{aligned}
$$

\footnotetext{
${ }^{7}$ as we learned after the previous installment of our work arXiv:1107.3834
} 
where the choices of signs at this stage are arbitrary but will come useful later on. The resulting quadratic form is thus calculated to

$$
q_{\widetilde{K}}=\left(\begin{array}{cc}
0 & \frac{1}{2} \\
\frac{1}{2} & 0
\end{array}\right)^{3}
$$

with the associated bilinear form taking values in $\mathbb{Q} / \mathbb{Z}$. An analogous analysis yields representatives $p_{i j} \in \widetilde{\Pi}^{*}$ of generators of $\widetilde{\Pi}^{*} / \widetilde{\Pi}$ which are glued to the $q_{i j}$ under an appropriate isomorphism

$$
\widetilde{\gamma}: \widetilde{K}^{*} / \widetilde{K} \longrightarrow \widetilde{\Pi}^{*} / \widetilde{\Pi}, \quad \widetilde{\gamma}\left(\overline{q_{i j}}\right)=\overline{p_{i j}},
$$

such that $q_{\widetilde{K}}=-q_{\widetilde{\Pi}} \circ \widetilde{\gamma}$ for the associated quadratic forms. In fact, we can use

$$
\begin{aligned}
& p_{12}:=\frac{1}{2}\left(f_{1}+f_{11}+f_{13}+f_{21}\right), \quad p_{34}:=\frac{1}{2}\left(f_{1}+f_{2}+f_{14}+f_{17}\right), \\
& p_{13}:=\frac{1}{2}\left(f_{1}+f_{11}+f_{14}+f_{16}\right), \quad p_{24}:=\frac{1}{2}\left(f_{1}+f_{13}+f_{17}+f_{18}\right), \\
& p_{14}:=\frac{1}{2}\left(f_{1}+f_{8}+f_{11}+f_{17}\right), \quad p_{23}:=\frac{1}{2}\left(f_{1}+f_{4}+f_{13}+f_{14}\right) .
\end{aligned}
$$

Denoting by $\widetilde{P}_{i j} \subset \mathcal{I}$ the sets of labels such that

$$
p_{i j}=\frac{1}{2} \sum_{n \in \widetilde{P}_{i j}} f_{n} \in \widetilde{\Pi}^{*}
$$

in (2.18) above, we find that the map $I$ of (2.14) maps $\widetilde{P}_{i j}$ to the plane $P_{i j} \subset \mathbb{F}_{2}^{4}$ given in (2.7). In other words, the isometry between $\Pi$ and $\widetilde{\Pi}(-1)$ induced by $I$ is compatible with the gluing prescriptions described in section 2.2 and above.

As to uniqueness of the embedding of the Kummer lattice in the Niemeier lattice $N(-1)$, we have

Proposition 2.3.5 Consider the Niemeier lattice $N$ of type $A_{1}^{24}$. The primitive embedding of the Kummer lattice $\Pi$ in $N(-1)$ found in proposition 2.3 .3 is unique up to automorphisms of $N$.

Proof: Assume that $i: \Pi \hookrightarrow N(-1)$ is a primitive embedding of the Kummer lattice $\Pi$ in $N(-1)$. Then from (2.5) we deduce that the $i\left(E_{\vec{a}}\right), \vec{a} \in \mathbb{F}_{2}^{4}$, yield 16 pairwise perpendicular vectors in $\widehat{\Pi}:=i(\Pi) \subset N(-1)$ on which the quadratic form takes value -2 . Hence by (2.9) and with the notations used there, for every $n \in \mathcal{I} \backslash \mathcal{O}_{9}$ we find some $\widehat{I}(n) \in \mathcal{I}$ such that $i\left(E_{I(n)}\right)= \pm f_{\widehat{I}(n)} \in \widehat{\Pi}$, where $I$ is the map (2.14) and the indices $\widehat{I}(n)$ are pairwise distinct. In particular, $i$ induces the hypercube structure of $\mathbb{F}_{2}^{4}$ on the labels $\left\{\widehat{I}(n) \mid n \in \mathcal{I} \backslash \mathcal{O}_{9}\right\}$, and the map induced by $i\left(E_{I(n)}\right) \mapsto f_{n}$ for all $n \in \mathcal{I} \backslash \mathcal{O}_{9}$ is an isometry $\widehat{\Pi} \cong \widetilde{\Pi}$.

Again by (2.5) we have $e:=\frac{1}{2} \sum_{\vec{a} \in \mathbb{F}_{2}^{4}} E_{\vec{a}} \in \Pi$ and thus $\frac{1}{2} \sum_{n \in \mathcal{I} \backslash \mathcal{O}_{9}} f_{\widehat{I}(n)} \in N$. However, by proposition 2.3.1 this implies that $\check{\mathcal{O}}:=\left\{\widehat{I}(n) \mid n \in \mathcal{I} \backslash \mathcal{O}_{9}\right\}$ gives a codeword of the Golay code. It follows that $\mathcal{O}:=\mathcal{I} \backslash \breve{\mathcal{O}}$ is an octad in the Golay code. This means that the lattice $\widehat{K}:=\widehat{\Pi}^{\perp} \cap N$ is isometric to the lattice $\widetilde{K}$ defined in (2.11). Note that $\widehat{K}$ is generated by the $f_{n}$ with $n \in \mathcal{O}$ along with $\frac{1}{2} \sum_{n \in \mathcal{O}} f_{n}$.

Using proposition 2.1.1 we can recover $N$ either from gluing $\widehat{K}$ to $\widehat{\Pi}$, or from gluing $\widetilde{K}$ to $\widetilde{\Pi}$. On the level of discriminant groups, our isometry $\widehat{\Pi} \cong \widetilde{\Pi}$ together with the respective gluing isometries yields an isometry of the discriminant groups $\widehat{K}^{*} / \widehat{K} \longrightarrow \widetilde{K}^{*} / \widetilde{K}$. We need to show that this isometry can be lifted to an isometry $\widehat{K} \longrightarrow \widetilde{K}$ in order to yield the isometry $\widehat{K} \oplus \widehat{\Pi} \cong \widetilde{K} \oplus \widetilde{\Pi}$ compatible with gluing. This can be done by explicit calculation:

Given the quadratic form (2.16) on our discriminant groups, it follows from the gluing prescription (2.17) that the preimages under $\widehat{K}^{*} / \widehat{K} \longrightarrow \widetilde{K}^{*} / \widetilde{K}$ of the $\overline{q_{i j}}$ with $q_{i j}$ given by (2.15) 
are of the form $\overline{\frac{1}{2} \sum_{n \in \widehat{Q}_{i j}} f_{n}}$, where $\frac{1}{2} \sum_{n \in \widehat{Q}_{i j}} f_{n} \in \widehat{K}^{*}$ is glued to $\frac{1}{2} \sum_{n \in \widehat{P}_{i j}} f_{n} \in \widehat{\Pi}^{*}$ with $\widehat{P}_{i j}=\widehat{I}\left(\widetilde{P}_{i j}\right)$ and $I\left(\widetilde{P}_{i j}\right)=P_{i j} \subset \mathbb{F}_{2}^{4}$ in (2.7). In other words, $\widehat{Q}_{i j} \subset \mathcal{O}$ is a quadruplet of labels such that $\widehat{P}_{i j} \cup \widehat{Q}_{i j}$ is an octad in the Golay code. Since $\mathcal{O}$ is an octad, by replacing $\widehat{Q}_{i j}$ by $\mathcal{O} \backslash \widehat{Q}_{i j}$ where necessary, we may assume that all $\widehat{Q}_{i j}$ share a common label, say $a$. Comparing to the $Q_{i j} \subset \mathcal{O}_{9}$ which in (2.15) yield $q_{i j}=\frac{1}{2} \sum_{n \in Q_{i j}}\left( \pm f_{n}\right)$, we map $f_{a} \mapsto f_{15}$. By means of symmetric differences, for example $\widehat{P}_{12}+\widehat{P}_{13}+\widehat{Q}_{12}+\widehat{Q}_{13}$ must yield an octad, where $\widehat{P}_{12}+\widehat{P}_{13}=\widehat{I}\left(\widetilde{P}_{12}+\widetilde{P}_{13}\right)$ is a quadruplet of labels. Thus $\widehat{Q}_{12}$ and $\widehat{Q}_{13}$ share precisely two entries, $a, b$, say. Again comparing to (2.15), we map $f_{b} \mapsto f_{6}$. Continuing in this fashion one obtains the desired isometry $\widehat{K} \longrightarrow \widetilde{K}$ which by construction induces $\widehat{K}^{*} / \widehat{K} \longrightarrow \widetilde{K}^{*} / \widetilde{K}$. In other words, it is compatible with gluing and we obtain an isometry $\widehat{K} \oplus \widehat{\Pi} \cong \widetilde{K} \oplus \widetilde{\Pi}$ which can be extended to a lattice automorphism $\alpha$ of $N$. Then $\alpha \circ i$ gives the primitive embedding of the Kummer lattice $\Pi$ in $N(-1)$ found in proposition 2.3.3.

Finally, we give yet another description of the Niemeier lattice $N$ in terms of the gluing techniques of proposition 2.1.1, which resembles the description of the full integral K3-homology in proposition 2.2.4

Proposition 2.3.6 Consider the Niemeier lattice $N$ of type $A_{1}^{24}$ with root sublattice $R$ generated by roots $f_{1}, \ldots, f_{24}$. With our choice (2.10) of a special octad $\mathcal{O}_{9}$ let $n_{0} \in \mathcal{O}_{9}$ and

$$
\begin{aligned}
& \widetilde{\mathcal{K}}_{n_{0}}:=\left\{\nu \in N \mid \forall n \notin \mathcal{O}_{9} \backslash\left\{n_{0}\right\}:\left\langle\nu, f_{n}\right\rangle=0\right\}, \\
& \widetilde{\mathcal{P}}_{n_{0}}:=\left\{\nu \in N \mid \forall n \in \mathcal{O}_{9} \backslash\left\{n_{0}\right\}:\left\langle\nu, f_{n}\right\rangle=0\right\} .
\end{aligned}
$$

Then $\widetilde{\mathcal{K}}_{n_{0}}$ is a root lattice of type $A_{1}^{7}$ and $\widetilde{\mathcal{P}}_{n_{0}}=\widetilde{\Pi} \oplus \operatorname{span}_{\mathbb{Z}}\left\{f_{n_{0}}\right\}$. Both lattices are primitive sublattices of $N$ which are orthogonal complements of one another. Furthermore, $\widetilde{\mathcal{K}}_{n_{0}}^{*} / \widetilde{\mathcal{K}}_{n_{0}} \cong$ $\widetilde{\mathcal{P}}_{n_{0}}^{*} / \widetilde{\mathcal{P}}_{n_{0}} \cong\left(\mathbb{Z}_{2}\right)^{7}$ under an isomorphism $\widetilde{g}_{n_{0}}: \widetilde{\mathcal{K}}_{n_{0}}^{*} / \widetilde{\mathcal{K}}_{n_{0}} \longrightarrow \widetilde{\mathcal{P}}_{n_{0}}^{*} / \widetilde{\mathcal{P}}_{n_{0}}$ with

$$
\begin{aligned}
& \widetilde{g}_{n_{0}}\left(\overline{q_{i j}}\right):=\frac{\widetilde{\gamma}\left(\overline{q_{i j}}\right) \text { for } i j=12,34,13,24,14,23,}{\frac{1}{2} f_{n_{0}}} ; \\
& \widetilde{g}_{n_{0}}\left(\frac{1}{2} \sum_{n \in \mathcal{O}_{9} \backslash\left\{n_{0}\right\}} f_{n}\right): \quad N \\
& \widetilde{\mathcal{P}}_{n_{0}} \cong \mathcal{P}(-1), \quad \cong\left\{(k, p) \in \widetilde{\mathcal{K}}_{n_{0}}^{*} \oplus \widetilde{\mathcal{P}}_{n_{0}}^{*} \mid \widetilde{g}_{n_{0}}(\bar{k})=\bar{p}\right\}
\end{aligned}
$$

with notations as in (2.15) $-(2.18)$.

Proof: The lattices $\widetilde{\mathcal{K}}_{n_{0}}, \widetilde{\mathcal{P}}_{n_{0}}$ are perpendicular primitive sublattices of $N$ of rank 7 and 17 and thus orthogonal complements of one another by construction.

By proposition 2.3.1 the lattice $\widetilde{\mathcal{K}}_{n_{0}} \subset \widetilde{\mathcal{K}}$ is generated by the $f_{n}$ with $n \in \mathcal{O}_{9} \backslash\left\{n_{0}\right\}$ along with linear combinations $\frac{1}{2} \sum_{n \in A} f_{n}$, if $A \subset \mathcal{O}_{9} \backslash\left\{n_{0}\right\}$ corresponds to a codeword of the Golay code. However, since $O_{9} \backslash\left\{n_{0}\right\}$ contains only 7 elements, while the shortest non-trivial codeword in the Golay code has weight 8 , we find $\widetilde{\mathcal{K}}_{n_{0}}=\operatorname{span}_{\mathbb{Z}}\left\{f_{n} \mid n \in \mathcal{O}_{9} \backslash\left\{n_{0}\right\}\right\}$, which is a root lattice of type $A_{1}^{7}$ as claimed.

Similarly, by (2.11) the lattice $\widetilde{\mathcal{P}}_{n_{0}}$ is generated by the elements of $\widetilde{\Pi}$ along with $f_{n_{0}}$ and any linear combination $\frac{1}{2} \sum_{n \in A} f_{n}$ with $A \cap \mathcal{O}_{9}=\left\{n_{0}\right\}$, if $A \subset \mathcal{I}$ corresponds to a codeword of the Golay code. However, since $\mathcal{O}_{9}$ corresponds to a codeword in the Golay code, any two codewords of which intersect in an even number of labels, no such $A$ can exist. In other words, $\widetilde{\mathcal{P}}_{n_{0}}=\widetilde{\Pi} \oplus \operatorname{span}_{\mathbb{Z}}\left\{f_{n_{0}}\right\}$, and thus $\widetilde{\mathcal{P}}_{n_{0}} \cong \mathcal{P}(-1)$ by proposition 2.3.4 along with the very definition of $\mathcal{P}$ in proposition 2.2.4.

Using proposition 2.1.1, the Niemeier lattice $N$ can be glued from $\widetilde{\mathcal{K}}_{n_{0}}$ and $\widetilde{\mathcal{P}}_{n_{0}}$ as claimed in (2.19). 


\section{The complex geometry and the symmetries of K3 surfaces}

In the preceding section 2.2, we have already addressed some properties of K3 surfaces and in particular of Kummer surfaces. Recall that the objective of this work is the investigation of finite groups of symplectic automorphisms of Kummer surfaces, and the role of the Mathieu group $M_{24}$ in their realisation. We need a number of additional techniques to describe and investigate symplectic automorphisms of Kummer surfaces. This section reviews some of these techniques and introduces new ones.

\subsection{Complex structures and dual Kähler classes}

Recall from definition 2.2.1 that we view a K3 surface $X$ as a complex surface, which thus in particular comes with a choice of complex structure. Moreover, $X$ has trivial canonical bundle and hence there exists a holomorphic $(2,0)$-form on $X$ which never vanishes and which represents a Hodge-de Rham class $\widehat{\Omega} \in H^{2}(X, \mathbb{C})$. Having worked in homology, so far, we introduce the 2-cycle $\Omega \in H_{2}(X, \mathbb{C})$ which is Poincaré dual to $\widehat{\Omega}$. By construction, it obeys $\Omega \vee \Omega=0$, and $H_{4}(X, \mathbb{R}) \ni \Omega \vee \bar{\Omega}$ is positive with respect to the intersection form $\langle\cdot, \cdot\rangle$ on $H_{*}(X, \mathbb{R})$. Decomposing $\Omega$ into its real and its imaginary part,

$$
\Omega=\Omega_{1}+i \Omega_{2}, \quad \Omega_{k} \in H_{2}(X, \mathbb{R}),
$$

the above conditions on $\Omega$ immediately imply

$$
\left\langle\Omega_{1}, \Omega_{2}\right\rangle=0, \quad\left\langle\Omega_{1}, \Omega_{1}\right\rangle=\left\langle\Omega_{2}, \Omega_{2}\right\rangle>0 .
$$

In other words, $\Omega_{1}, \Omega_{2} \in H_{2}(X, \mathbb{R})$ form an orthogonal basis of a positive definite oriented 2dimensional subspace of $H_{2}(X, \mathbb{R})$, which is traditionally denoted by $\Omega$, too. While the choice of complex structure on $X$ obviously determines the position of $\Omega$ relative to the lattice of integral homology $H_{2}(X, \mathbb{Z})$, it is a deep theorem, which is equivalent to the GLOBAL TORELLI THEOREM FOR K3 SURFACES [32, 33, 34, 35, 36, that the converse is also true:

Theorem 3.1.1 (Torelli Theorem) Consider a K3 surface $X$ and the 2-dimensional oriented subspace $\Omega$ of $H_{2}(X, \mathbb{R})$ whose basis is represented by the real and imaginary part of the Poincaré dual of a holomorphic $(2,0)$-form on $X$ which vanishes nowhere. The complex structure of $X$ is uniquely determined by the position of $\Omega$ relative to the lattice $H_{2}(X, \mathbb{Z})$ of integral homology.

By the Torelli Theorem 3.1.1, the moduli space $\mathcal{M}_{c p x}$ of complex structures on a K3 surface is the Grassmannian $\widetilde{\mathcal{M}}_{c p x}$ of positive definite oriented 2-dimensional subspaces $\Omega$ of $\mathbb{R}^{3,19}=$ $\left(U^{3} \oplus E_{8}^{2}(-1)\right) \otimes \mathbb{R}$, modulo the action of the automorphism group of $U^{3} \oplus E_{8}^{2}(-1)$. This moduli space is equipped with its natural topology, which however does not have the Hausdorff property [37]. By choosing a marking $H_{2}(X, \mathbb{Z}) \cong U^{3} \oplus E_{8}^{2}(-1), H_{*}(X, \mathbb{Z}) \cong U^{4} \oplus E_{8}^{2}(-1)$, we work in the smooth connected cover $\widetilde{\mathcal{M}}_{c p x}$ of the moduli space. This allows us to explicitly specify the complex structure of a given K3 surface in our applications by writing out the basis $\Omega_{1}, \Omega_{2}$ of $\Omega$ in terms of lattice vectors in $H_{2}(X, \mathbb{Z})$. Indeed, thereby we specify the very location of $\Omega$ relative to $H_{2}(X, \mathbb{Z})$ and thus the complex structure, by theorem 3.1.1.

In terms of local holomorphic coordinates $z_{1}, z_{2}$, the procedure works as follows: Locally, a holomorphic (2,0)-form representing $\widehat{\Omega}$ has the form $d z_{1} \wedge d z_{2}$, which with respect to real coordinates $\vec{x}=\left(x_{1}, x_{2}, x_{3}, x_{4}\right), z_{1}=x_{1}+i x_{2}, z_{2}=x_{3}+i x_{4}$, as before, yields

$$
d z_{1} \wedge d z_{2}=\left[d x_{1} \wedge d x_{3}-d x_{2} \wedge d x_{4}\right]+i\left[d x_{1} \wedge d x_{4}+d x_{2} \wedge d x_{3}\right]=: \widehat{\Omega}_{1}+i \widehat{\Omega}_{2}
$$


Here, the real valued two-forms $\widehat{\Omega}_{1}, \widehat{\Omega}_{2}$ represent the Poincaré duals of $\Omega_{1}, \Omega_{2}$. Hence with respect to standard real coordinate vector fields $\vec{e}_{1}, \ldots, \vec{e}_{4}$, the latter are readily identified as

$$
\Omega_{1}=e_{1} \vee e_{3}-e_{2} \vee e_{4}, \quad \Omega_{2}=e_{1} \vee e_{4}+e_{2} \vee e_{3} .
$$

Recall from definition 2.2 .2 that a Kummer surface $X$ with underlying torus $T(\Lambda)$ carries the complex structure induced from the universal cover $\mathbb{C}^{2} \cong \mathbb{R}^{4}$ of $T(\Lambda)$. Moreover, as remarked at the end of section 2.2 , the Kummer construction provides us with a natural marking $H_{2}(X, \mathbb{Z}) \cong U^{3} \oplus E_{8}^{2}(-1), H_{*}(X, \mathbb{Z}) \cong U^{4} \oplus E_{8}^{2}(-1)$, which we use throughout our discussion of Kummer surfaces. In other words, by the above-mentioned procedure we calculate the complex structure of the Kummer surface $X=\widetilde{T(\Lambda) / \mathbb{Z}_{2}}$ in terms of the lattice data $\Lambda \subset \mathbb{C}^{2} \cong \mathbb{R}^{4}$, where the generators $\vec{\lambda}_{1}, \ldots, \vec{\lambda}_{4}$ of $\Lambda$ are part of the data. Indeed, $\vec{\lambda}_{1}, \ldots, \vec{\lambda}_{4}$ are expressed in terms of the standard basis vectors $\vec{e}_{1}, \ldots, \vec{e}_{4}$ of $\mathbb{R}^{4}$. Thus we obtain expressions for the Poincaré duals of $d x_{1} \wedge d x_{3}-d x_{2} \wedge d x_{4}$ and $d x_{1} \wedge d x_{4}+d x_{2} \wedge d x_{3}$ in terms of the $\lambda_{i j}=\lambda_{i} \vee \lambda_{j}$, our standard generators of $H_{2}(T(\Lambda), \mathbb{Z})$. Now recall from definition 2.2.2 the linear map $\pi_{*}: H_{2}(T(\Lambda), \mathbb{R}) \longrightarrow H_{2}(X, \mathbb{R})$ which is induced by the rational map $\pi: T(\Lambda)-\rightarrow$ $X$. The images of the Poincaré duals of $d x_{1} \wedge d x_{3}-d x_{2} \wedge d x_{4}$ and $d x_{1} \wedge d x_{4}+d x_{2} \wedge d x_{3}$ under $\pi_{*}$ yield the two-cycles $\Omega_{1}, \Omega_{2}$ specifying the complex structure of the Kummer surface $X$. One thus immediately obtains expressions for the $\Omega_{k}$ in terms of the lattice $H_{2}(X, \mathbb{Z})$, uniquely specifying the complex structure of $X$. Note that this procedure allows us to vary the underlying lattice $\Lambda$ of $T(\Lambda)=\mathbb{C}^{2} / \Lambda$ and thereby the induced complex structure of $X=T \widetilde{T(\Lambda) / \mathbb{Z}_{2}}$, giving the relative position of $\Omega=\operatorname{span}_{\mathbb{R}}\left\{\Omega_{1}, \Omega_{2}\right\}$ in $H_{2}(X, \mathbb{R})=H_{2}(X, \mathbb{Z}) \otimes \mathbb{R}$ in terms of $H_{2}(X, \mathbb{Z})$ with our choice of marking. Here, the lattice $H_{2}(X, \mathbb{Z})$ remains fixed, while the position of $\Omega$ varies with $\Lambda$, thus describing a path in the smooth connected cover $\widetilde{\mathcal{M}}_{c p x}$ of the moduli space of complex structures on K3.

Example: Consider the standard SQUARE TORUs $T_{0}:=T\left(\mathbb{Z}^{4}\right)=\mathbb{C}^{2} / \mathbb{Z}^{4}$, where we simply have $\vec{e}_{i}=\vec{\lambda}_{i}, i=1, \ldots, 4$, and thus $\Omega_{1}=\pi_{*} \lambda_{13}-\pi_{*} \lambda_{24}, \Omega_{2}=\pi_{*} \lambda_{14}+\pi_{*} \lambda_{23} \in H_{2}(X, \mathbb{Z})$. Hence the Kummer surface $X_{0}$ with underlying torus $T_{0}$ has the special property that the 2-dimensional space $\Omega \subset H_{2}\left(X_{0}, \mathbb{R}\right)$ which specifies its complex structure contains a sublattice of $H_{2}\left(X_{0}, \mathbb{Z}\right)$ of (the maximal possible) rank 2. For such K3 surfaces, by a seminal result of Shioda and Inose [38], the quadratic form of the TRANSCENDENTAL LATTICE $\Omega \cap H_{2}(X, \mathbb{Z})$ already uniquely determines the complex structure of $X$. In other words, the complex structure of the Kummer surface $X_{0}$ with underlying torus $T_{0}$ is uniquely determined by the following quadratic form of its transcendental lattice:

$$
\left(\begin{array}{ll}
4 & 0 \\
0 & 4
\end{array}\right)
$$

According to the final remark of [38], this means that $X_{0}$ agrees with the so-called ELLIPTIC MODULAR SURFACE OF LEVEL 4 defined over $\mathbb{Q}(\sqrt{-1})$ of [39, p. 57].

By a deep result due to Siu [35], every K3 surface is KäHLER. In fact, in addition to a complex structure $\Omega \subset H_{2}(X, \mathbb{R})$, we always fix a KäHLER CLASS on each of our K3 surfaces $X$. By definition, a Kähler class is the cohomology class of the two-form which is associated to a Kähler metric on $X$. By [40] this amounts to choosing a real, positive, effective element of $H^{1,1}(X, \mathbb{C})$. Under Poincaré duality, this translates into the choice of some $\omega \in \Omega^{\perp} \cap H_{2}(X, \mathbb{R})$ with $\langle\omega, \omega\rangle>0$, ensuring effectiveness by replacing $\omega$ by $-\omega$ if necessary.

Definition 3.1.2 Consider a K3 surface $X$, and let $\Omega \subset H_{2}(X, \mathbb{R})$ denote the oriented 2dimensional subspace which specifies the complex structure of $X$ according to the Torelli Theorem 3.1.1. A choice of DUAL KäHLER CLASS on $X$ is the choice of some $\omega \in \Omega^{\perp} \cap H_{2}(X, \mathbb{R})$ with $\langle\omega, \omega\rangle>0$. If the dual Kähler class obeys $\omega \in H_{2}(X, \mathbb{Z})$, then $\omega$ is called a POLARIZATION of $X$. 
If $X$ is a Kummer surface with underlying torus $T(\Lambda)$, let $\omega_{T}$ denote the Poincaré dual of the standard Kähler class induced from the standard Euclidean metric on $\mathbb{C}^{2}$. Then with $\pi_{*}$ as in definition 2.2.2, we call $\pi_{*} \omega_{T} \in \Omega^{\perp} \cap H_{2}(X, \mathbb{R})$ the INDUCED DUAL KÄHLER CLASS ON $X$.

By the above, a choice of a dual Kähler class is equivalent to the choice of a Kähler structure on $X$, and the choice of a polarization is equivalent to the choice of a Kähler structure which is represented by an integral Kähler form. It is known that a K3 surface $X$ is ALGEBRAIC, that is, $X$ can be viewed as a complex subvariety of some complex projective space $\mathbb{P}^{N}$, if and only if it admits the choice of a polarization (see e.g. [41, pp. 163, 191]).

We equip all Kummer surfaces with the dual Kähler class induced from its underlying torus. That this special type of dual Kähler class has been chosen is an important assumption which we make throughout our work. Without loss of generality we are also assuming a coordinate description for all our tori $T=T(\Lambda)=\mathbb{C}^{2} / \Lambda$ where the dual Kähler class $\omega_{T}$ of $T$ is induced from the standard Kähler structure of $\mathbb{C}^{2}$. The Kähler class dual to $\pi_{*} \omega_{T}$ is actually located on a wall of the Kähler cone of $X$. In other words, it represents a degenerate (or ORBIFOLD LIMIT of a) Kähler metric on $X$. In this sense, the induced (dual) Kähler classes on our Kummer surfaces yield DEGENERATE KÄHLER STRUCTURES.

For later convenience, note that in terms of the standard local holomorphic coordinates on $T(\Lambda)=\mathbb{C}^{2} / \Lambda$ the standard Kähler class is represented by

$$
\frac{1}{2 i}\left(d z_{1} \wedge d \bar{z}_{1}+d z_{2} \wedge d \bar{z}_{2}\right)=d x_{1} \wedge d x_{2}+d x_{3} \wedge d x_{4}
$$

and hence

$$
\omega=\pi_{*} \omega_{T}=e_{1} \vee e_{2}+e_{3} \vee e_{4}
$$

with notations as above. The induced dual Kähler class on the Kummer surface obtained from $T(\Lambda)$ can thus be immediately calculated in terms of the lattice $H_{2}(X, \mathbb{Z})$ and our fixed marking, given the lattice $\Lambda$ of the underlying torus.

Example: For our square torus $T_{0}=T\left(\mathbb{Z}^{4}\right)$ above we argued that we have $\omega=\pi_{*} \omega_{T}=$ $\pi_{*} \lambda_{12}+\pi_{*} \lambda_{34} \in H_{2}\left(X_{0}, \mathbb{Z}\right)$ for the associated Kummer surface $X_{0}$. Hence this Kummer surface is algebraic. The real 3 -dimensional subspace $\Sigma$ of $H_{2}\left(X_{0}, \mathbb{R}\right)$ containing $\Omega$ and $\omega$ has the property that $\Sigma \cap H_{2}\left(X_{0}, \mathbb{Z}\right)$ yields a lattice of (the maximal possible) rank 3 , with quadratic form

$$
\left(\begin{array}{lll}
4 & 0 & 0 \\
0 & 4 & 0 \\
0 & 0 & 4
\end{array}\right) .
$$

If $X$ denotes a K3 surface with complex structure specified by $\Omega \subset H_{2}(X, \mathbb{R})$ as in the Torelli Theorem 3.1.1 and with dual Kähler class $\omega$ according to definition 3.1.2, then the oriented 3 -dimensional subspace $\Sigma$ of $H_{2}(X, \mathbb{R})$ containing $\Omega$ and $\omega$ uniquely determines a real Einstein metric on $X$, up to its volume, or equivalently a hyperkähler structure on $X$ (see for example [42, 43] for a review). Vice versa, $\Sigma$ is uniquely determined by such a hyperkähler structure. The moduli space $\mathcal{M}_{h k}$ of hyperkähler structures on $X$ is thus the Grassmannian $\widetilde{\mathcal{M}}_{h k}$ of positive definite oriented 3 -dimensional subspaces $\Sigma$ of $\mathbb{R}^{3,19}=\left(U^{3} \oplus E_{8}^{2}(-1)\right) \otimes \mathbb{R}$, modulo the action of the automorphism group of $U^{3} \oplus E_{8}^{2}(-1)$. This moduli space carries a natural Hausdorff topology. By choosing a fixed marking, as we are doing in this work, we are effectively working in the smooth connected cover $\widetilde{\mathcal{M}}_{h k}$ of this moduli space. A smooth variation of the generators $\vec{\lambda}_{1}, \ldots, \vec{\lambda}_{4}$ of the underlying lattice $\Lambda$ for Kummer surfaces $T \widetilde{(\Lambda) / \mathbb{Z}_{2}}$ thus amounts to the description of a smooth path in $\widetilde{\mathcal{M}}_{h k}$, which we simply call a KUMmer PATH. 


\subsection{Holomorphic symplectic automorphisms of K3 surfaces}

In this subsection, we consider a K3 surface $X$ with a complex structure that is encoded in terms of a real 2-dimensional oriented positive definite subspace $\Omega \subset H_{2}(X, \mathbb{R})$ according to the Torelli Theorem 3.1.1. We discuss the notion of SYMPLECTIC AUTOMORPhism 8 and HOLOMORPHIC SYMPLECTIC AUTOMORPHISMS of $X$ :

Definition 3.2.1 Consider a K3 surface $X$. A map $f: X \longrightarrow X$ of finite order is called a SYMPLECTIC AUTOMORPHISM if and only if $f$ is biholomorphic and the induced map $f_{*}: H_{*}(X, \mathbb{R}) \longrightarrow H_{*}(X, \mathbb{R})$ leaves the complex structure $\Omega \subset H_{*}(X, \mathbb{R})$ invariant.

If $\omega$ is a dual Kähler class on $X$ and $f_{*} \omega=\omega$, then $f$ is a HOLOMORPHIC SYMPLECTIC AUTOMORPHISM with respect to $\omega$.

When a dual Kähler class $\omega$ on $X$ has been specified, then the group of holomorphic symplectic automorphisms of $X$ with respect to $\omega$ is called the SYMMETRY GROUP of $X$.

Consider a map $f: X \longrightarrow X$ of finite order which is bijective, such that both $f$ and $f^{-1}$ respect the complex structure of $X$. The induced linear map $f_{*}$ on $H_{*}(X, \mathbb{R})$ restricts to a lattice automorphism on $H_{2}(X, \mathbb{Z})$ and, according to our description of complex structures by means of the Torelli Theorem 3.1.1, it acts as a multiple of the identity on $\Omega$. By definition 3.2.1, $f$ is a symplectic automorphism if and only if $f_{*}$ acts as the identity on $\Omega$. In fact, by another version of the Torelli Theorem (see e.g. [11, Thm. 2.7']) the restriction of $f_{*}$ to $H_{2}(X, \mathbb{Z}$ ) (also denoted $f_{*}$ ) uniquely determines the symplectic automorphism $f$. The following version of the Torelli Theorem is most adequate for our applications to symplectic automorphisms, and it is obtained from [11, Thms. 2.7'\&4.3] in conjunction with [32] and [44, Prop. VIII.3.10]:

Theorem 3.2.2 (Torelli Theorem) Consider a K3 surface $X$ and a lattice automorphism $\alpha$ of $\mathrm{H}_{2}(X, \mathbb{Z})$, i.e. a linear map which respects the intersection form. Assume that after linear extension to $H_{2}(X, \mathbb{R}), \alpha$ leaves $\Omega$ invariant.

Then $\alpha$ is induced by a symplectic automorphism of $X$ if and only if the following holds: $\alpha$ preserves effectiveness for every $E \in \Omega^{\perp} \cap H_{2}(X, \mathbb{Z})$ with $\langle E, E\rangle=-2$, the invariant sublattice $L^{\alpha}:=H_{2}(X, \mathbb{Z})^{\alpha}=\left\{v \in H_{2}(X, \mathbb{Z}) \mid \alpha v=v\right\}$ has a negative definite orthogonal complement $L_{\alpha}:=\left(L^{\alpha}\right)^{\perp} \cap H_{2}(X, \mathbb{Z})$, and for all $v \in L_{\alpha},\langle v, v\rangle \neq-2$.

In this case the symplectic automorphism $f$ with $\alpha=f_{*}$ is uniquely determined.

This theorem allows us to carry out the entire discussion of symplectic automorphisms of a $\mathrm{K} 3$ surface $X$ in terms of lattice automorphisms on $H_{*}(X, \mathbb{Z})$. To treat finite symplectic automorphism groups, we

Remark 3.2.3 If $G$ is a finite group of symplectic automorphisms of a $K 3$ surface $X$, let $L_{G}:=\left(L^{G}\right)^{\perp} \cap H_{2}(X, \mathbb{Z})$ denote the orthogonal complement of the lattice $L^{G} \subset H_{*}(X, \mathbb{Z})$ which is pointwise invariant under the induced action of $G$ on $H_{*}(X, \mathbb{Z})$, generalizing the lattice $L_{\alpha}$ in the above version of the Torelli Theorem [3.2.2. By [11, Lemma 4.2], the lattice $L_{G}$ is negative definite. To see why this only follows when $G$ is finite, note that the proof given in [11] uses the resolution of the quotient $X / G$. Indeed, by our assumptions on $G$, all singularities of $X / G$ are isolated, and the minimal resolution of all its singularities yields a K3 surface $Y$. Moreover, in [1] Nikulin proves $L_{G} \otimes \mathbb{Q} \cong M_{Y} \otimes \mathbb{Q}$ with $M_{Y}$ the sublattice of $\mathrm{H}_{2}(Y, \mathbb{Z})$ generated by the exceptional classes in the resolution. Since $M_{Y}$ is negative definite by [45], the claim follows.

\footnotetext{
${ }^{8}$ Here, we follow the slightly misleading terminology which has become standard, by now. Note however that in Nikulin's original work such automorphisms are called ALGEBRAIC [11, Def. 0.2], and that the definition of symplectic automorphisms does not refer to a symplectic structure on $X$.
} 
Now the restriction to finite symplectic automorphism groups of K3 surfaces conveniently translates into the restriction to symplectic automorphism groups which preserve some dual Kähler class:

Proposition 3.2.4 Consider a $K 3$ surface $X$, and denote by $G$ a non-trivial group of symplectic automorphisms of $X$.

Then $G$ is finite if and only if $X$ possesses a dual Kähler class which is invariant under $G$. Equivalently, the sublattice $L^{G} \subset H_{*}(X, \mathbb{Z})$ which is pointwise invariant under the induced action of $G$ on $H_{*}(X, \mathbb{Z})$ has signature $\left(4, m_{-}\right)$with $m_{-} \in \mathbb{N}$ and $m_{-} \geq 1$.

If $X$ is an algebraic $K 3$ surface, then $G$ is finite if and only if $X$ possesses a polarization which is invariant under $G$.

Proof: Consider the PiCARD Lattice $\operatorname{Pic}(X):=\Omega^{\perp} \cap H_{2}(X, \mathbb{Z})$ of rank $\rho \in \mathbb{N}, 0 \leq \rho \leq 20$, and its orthogonal complement $T_{X}:=\operatorname{Pic}(X)^{\perp} \cap H_{2}(X, \mathbb{Z})$. Then by 11, Lemma 4.2], the lattice $H_{0}(X, \mathbb{Z}) \oplus T_{X} \oplus H_{4}(X, \mathbb{Z})$ is a sublattice of $L^{G}$, so in particular $\Omega \subset\left(L^{G} \cap H_{2}(X, \mathbb{Z})\right) \otimes \mathbb{R}$.

Assume first that $G$ is finite. Then by Remark 3.2.3, the lattice obtained as $L_{G}:=\left(L^{G}\right)^{\perp} \cap$ $H_{2}(X, \mathbb{Z})$ is negative definite, such that $L^{G}$ is a lattice of signature $\left(4, m_{-}\right)$for some $m_{-} \in \mathbb{N}$. Since $H_{0}(X, \mathbb{Z}) \oplus H_{4}(X, \mathbb{Z}) \subset L^{G}$, we have $m_{-} \geq 1$ as claimed.

Next assume that $L^{G}$ has signature $\left(4, m_{-}\right)$with $m_{-} \geq 1$. Recall from the above that $\Omega \subset\left(L^{G} \cap H_{2}(X, \mathbb{Z})\right) \otimes \mathbb{R}$, where $H_{0}(X, \mathbb{Z}) \oplus H_{4}(X, \mathbb{Z}) \subset L^{G}$ implies that $L^{G} \cap H_{2}(X, \mathbb{Z})$ has signature $\left(3, m_{-}-1\right)$, while $\Omega$ is positive definite of dimension 2 . Hence there exists some $\omega \in\left(L^{G} \cap H_{2}(X, \mathbb{Z})\right) \otimes \mathbb{R}$ with $\omega \in \Omega^{\perp}$ and $\langle\omega, \omega\rangle>0$. In other words, $X$ possesses a dual Kähler class which is invariant under $G$.

Conversely, assume that $X$ possesses a dual Kähler class $\omega$ which is invariant under $G$. Hence the induced action of $G$ on $H_{*}(X, \mathbb{R})$ leaves the 3 -dimensional vector space $\Sigma \subset H_{*}(X, \mathbb{R})$ generated by $\Omega$ and $\omega$ invariant. Thus the non-trivial action of $G$ on $H_{*}(X, \mathbb{Z})$ amounts to an action on the negative definite lattice $\Sigma^{\perp} \cap H_{2}(X, \mathbb{Z})$. It is therefore given by a permutation group on every set of vectors of same length in that lattice, a finite set for every length. Thus $G$ is represented on $H_{*}(X, \mathbb{R})$ by the action of a finite group. Moreover, the uniqueness statement in the Torelli Theorem 3.2 .2 implies that $G$ acts faithfully on $H_{*}(X, \mathbb{R})$. It follows that $G$ is finite.

Now assume that $X$ is algebraic. Since by definition 3.1.2 every polarization of $X$ is a dual Kähler class, it remains to be shown that there exists a polarization $\omega$ fixed by $G$ if $L^{G}$ has signature $\left(4, m_{-}\right)$with $m_{-} \geq 1$. Indeed, if $X$ is algebraic, then $\operatorname{Pic}(X)$ has signature $(1, \rho-1)$ with $1 \leq \rho \leq 20$, and thus its orthogonal complement $T_{X}$ has signature $(2,20-\rho)$. Moreover, by the above the lattice $H_{0}(X, \mathbb{Z}) \oplus T_{X} \oplus H_{4}(X, \mathbb{Z})$ of signature $(3,21-\rho)$ is a sublattice of $L^{G}$. Hence for $L^{G}$ of signature $\left(4, m_{-}\right), m_{-} \geq 1$, there exists some $\omega \in L^{G}$ with $\langle\omega, \omega\rangle>0$ and $\omega \in T_{X}^{\perp} \cap H_{2}(X, \mathbb{Z}) \subset \Omega^{\perp}$. In other words, there exists a polarization of $X$ which is invariant under $G$.

In this work, we restrict our attention to the investigation of finite symplectic automorphism groups of K3 surfaces $X$. Hence by theorem 3.2.4 there exists a dual Kähler class $\omega$ of $X$ which is invariant under the action of $G$. Once a complex structure $\Omega$ and dual Kähler class $\omega$ of our K3 surface have been chosen, the group $G$ of all holomorphic symplectic automorphisms with respect to $\omega$ is uniquely determined. By the above, it solely depends on the relative position of the 3 -dimensional space $\Sigma \subset H_{*}(X, \mathbb{R})$ generated by $\Omega$ and $\omega$ with respect to the lattice $H_{*}(X, \mathbb{Z})$. As explained at the end of section 3.1, $\Sigma$ uniquely determines a hyperkähler structure on $X$ and is uniquely determined by such a hyperkähler structure. Working with a fixed marking, our study of finite symplectic automorphism groups of K3 surfaces thus is naturally carried out on the smooth connected cover $\widetilde{\mathcal{M}}_{h k}$ of the moduli space of hyperkähler 
structures on K3.

Mukai shows in 9 that the action of a finite symplectic automorphism group $G$ on $H_{*}(X, \mathbb{Q})$ is a Mathieu Representation, that is: The character $\mu$ of this representation is given by

$$
\mu(g)=24\left(\operatorname{ord}(g) \prod_{p \mid \operatorname{ord}(g)}\left(1+\frac{1}{p}\right)\right)^{-1} \quad \forall g \in G .
$$

Furthermore, with $M_{23} \subset M_{24}$ the stabilizer group in the Mathieu group $M_{24}$ of one label in $\mathcal{I}=\{1, \ldots, 24\}$, Mukai found the following seminal result [9]:

Theorem 3.2.5 Consider a K3 surface $X$, and a finite group $G$ of symplectic automorphisms of $X$. Then $G$ is isomorphic to a subgroup of one of those 11 subgroups of $M_{23}$ which decompose $\mathcal{I}$ into at least five orbits.

Following Mukai, two further independent proofs of the above theorem were given, namely by Xiao [46] and by Kondo [10], see also [47]. We use a number of ideas from Kondo's proof throughout this work. Therefore, we briefly review its main steps, adjusting it slightly to fit our purposes:

Proof (sketch): Assume that $X$ is a $\mathrm{K} 3$ surface and that $G$ is a non-trivial finite group acting as symplectic automorphism group on $X$. Let $L^{G} \subset H_{*}(X, \mathbb{Z})$ denote the invariant sublattice of the integral homology 9 of $X$ and $L_{G}:=\left(L^{G}\right)^{\perp} \cap H_{*}(X, \mathbb{Z})$. By proposition 3.2.4, $L_{G}$ is negative definite of rank at most 19 , while $L^{G}$ has at least rank 5. Moreover, denoting by $v_{0}, v$ a choice of generators of $H_{0}(X, \mathbb{Z}), H_{4}(X, \mathbb{Z})$ with $\left\langle v_{0}, v\right\rangle=1$, we have a lattice vector $v_{0}-v \in L^{G}$ on which the quadratic form takes value -2 .

As an application of theorem 2.1.3, Kondo proves that a lattice $N_{G} \oplus\langle 2\rangle$ isometric to $\left(L_{G} \oplus\right.$ $\langle-2\rangle)(-1)$ can be primitively embedded in some Niemeier lattice $\widetilde{N}$ (see definition 2.0.3), where $\langle 2\rangle$ denotes a lattice of rank 1 with quadratic form (2) on a generator $f$, and $\langle-2\rangle=$ $\langle 2\rangle(-1)$. In fact, since $G$ acts trivially on $L^{G}$, it acts trivially on the discriminant group $\left(L^{G}\right)^{*} / L^{G}$. This in turn implies a trivial action of $G$ on the discriminant group of $L_{G}$, since $H_{*}(X, \mathbb{Z})$ is obtained by the gluing techniques of proposition 2.1.1 from $L^{G}$ and $L_{G}$. Hence on the Niemeier lattice $\widetilde{N}$, which can be obtained by the gluing techniques from $N_{G} \cong L_{G}(-1)$ and its orthogonal complement $N^{G}:=\left(N_{G}\right)^{\perp} \cap \widetilde{N}$, the action of $G$ on $N_{G} \cong L_{G}(-1)$ can be extended to $\widetilde{N}$, leaving $N^{G}$ invariant (see [11, Prop. 1.1]). Note that in particular, by construction, the invariant sublattice $N^{G}$ of $\widetilde{N}$ has $\operatorname{rank} \operatorname{rk}\left(N^{G}\right)=\operatorname{rk}\left(L^{G}\right) \geq 5$, and it contains the vector $f$ on which the quadratic form takes value 2 . While $N^{G}$ and $L^{G}$ in general have little in common, apart from their ranks and their discriminant groups, note that we can naturally identify $10 \in N^{G}$ with $v_{0}-v \in L^{G} \subset H_{*}(X, \mathbb{Z})$.

Next, for each Niemeier lattice $\widetilde{N}$ with root sublattice $\widetilde{R}$, Kondo shows that the induced action on $\widetilde{N} / \widetilde{R}$ gives an injective image of the $G$-action and that it yields an embedding of $G$ in $M_{23}$. The latter is readily seen in the case of the Niemeier lattice $N$ of type $A_{1}^{24}$ : Here, $N / R \cong \mathcal{G}_{24} \subset \mathbb{F}_{2}^{24}$ with $\mathcal{G}_{24}$ the Golay code by proposition 2.3.1. Hence the action of $G$ yields a group of automorphisms of the Golay code. Since $M_{24}$ is the automorphism group of $\mathcal{G}_{24}$, this yields an embedding of $G$ in the Mathieu group $M_{24}$. Moreover, the invariant part $N^{G}$ of $N$ by construction contains the root $f$. Hence the induced action of $G$ on the Golay code stabilizes the corresponding label in $\mathcal{I}$. Therefore, Kondo's construction indeed embeds $G$ in

\footnotetext{
${ }^{9}$ Here, we slightly modify Kondo's conventions: First, we work in homology instead of cohomology, which by Poincaré duality is equivalent. Second, instead of restricting to $H_{2}(X, \mathbb{Z})$ we consider the total integral K3homology, such that our lattice $L^{G}$ differs from the one in Kondo's work by a summand $H_{0}(X, \mathbb{Z}) \oplus H_{4}(X, \mathbb{Z}) \cong$ $U$, a hyperbolic lattice. Since the latter is unimodular, the arguments carry through identically.

${ }^{10}$ To obtain such an interpretation for $f$, our modification of Kondo's conventions is crucial.
} 
the subgroup $M_{23} \subset M_{24}$ which stabilizes that label.

In fact, by Mukai's appendix to Kondo's paper, the Niemeier lattice $N$ of type $A_{1}^{24}$ can be used to construct a symplectic action of each of the 11 groups $G$ in Mukai's classification. Note that this does not imply that the Niemeier lattices $\widetilde{N}$ can be replaced by $N$ within the above proof. Mukai also proves in [9] that each of the 11 groups in his classification actually occurs as the symplectic automorphism group of some algebraic K3 surface. The largest of these subgroups of $M_{23}$ is the polarization-preserving symplectic automorphism group $M_{20}$ of a particular deformation of the Fermat quartic, a group of order 960.

\subsection{Holomorphic symplectic automorphisms of Kummer surfaces}

Throughout this subsection we assume that $X$ is a Kummer surface with underlying torus $T=T(\Lambda), \Lambda \subset \mathbb{C}^{2}$. According to definition 2.2.2, $X$ carries the complex structure which is induced from the universal cover $\mathbb{C}^{2}$ of $T$. Furthermore, according to definition 3.1.2, we use the induced dual Kähler class on $X$. We are interested in the group of holomorphic symplectic automorphisms of $X$ with respect to that dual Kähler class. It is important to keep in mind that we are making a very special choice of dual Kähler class, which severely restricts the types of symmetry groups that are accessible to our methods. In this subsection, we determine the generic structure of such holomorphic symplectic automorphism groups of Kummer surfaces. While the description of these groups themselves, which we give in proposition 3.3.4, along with the ideas that lead to it are known to the experts, our observation about their action on the K3-homology in theorem 3.3.7 is new.

We begin by specifying a group of holomorphic symplectic automorphisms that all our Kummer surfaces share:

Proposition 3.3.1 Consider a Kummer surface $X$ with underlying torus $T$, equipped with the induced dual Kähler class. Let $G$ denote the holomorphic symplectic automorphism group of $X$ with respect to that dual Kähler class. Then $G$ contains an abelian subgroup $G_{t}$ which is isomorphic to $\left(\mathbb{Z}_{2}\right)^{4}$. With notations as in definition 2.2 .2 and proposition 2.2 .3 ,

$$
G_{t}=\left\{t^{\vec{b}} \mid \vec{b} \in \mathbb{F}_{2}^{4}\right\}
$$

where each $\alpha^{\vec{b}}:=t_{*}^{\vec{b}}$ acts trivially on $K=\pi_{*}\left(H_{2}(T, \mathbb{Z})\right)$, and its action on the Kummer lattice $\Pi$ is induced by $E_{\vec{a}} \mapsto E_{\vec{a}+\vec{b}}$ for all $\vec{a} \in \mathbb{F}_{2}^{4}$.

Proof: From proposition 2.2 .3 it immediately follows that the action of each $\alpha^{\vec{b}}$ described above induces a lattice automorphism on $H_{*}(X, \mathbb{Z})$, such that for the sublattice $L^{\alpha^{\vec{b}}}$ of $H_{2}(X, \mathbb{Z})$ which is invariant under $\alpha^{\vec{b}}$ we have $L^{\alpha^{\vec{b}}} \supset K$. It follows that $L_{\alpha^{\vec{b}}}:=\left(L^{\alpha^{\vec{b}}}\right)^{\perp} \cap$ $H_{2}(X, \mathbb{Z})$ obeys $L_{\alpha^{\vec{b}}} \subset \Pi$. Thus $L_{\alpha^{\vec{b}}}$ is negative definite. Moreover, by construction $\alpha^{\vec{b}}$ preserves effectiveness, and since $e:=\frac{1}{2} \sum_{\vec{a} \in \mathbb{F}_{2}^{4}} E_{\vec{a}} \in L^{\alpha^{\vec{b}}}$ and $L_{\alpha^{\vec{b}}}=\left(L^{\alpha^{\vec{b}}}\right)^{\perp} \cap \Pi,\langle v, v\rangle \neq-2$ for all $v \in L_{\alpha^{b}}$. Hence by the Torelli Theorem $\left[3.2 .2, \alpha^{\vec{b}}\right.$ is indeed induced by a uniquely determined holomorphic symplectic automorphism $t^{\vec{b}}$ of $X$.

With $T=T(\Lambda)$, recall from (2.4) and definition 2.2 .2 that each $E_{\vec{a}}, \vec{a} \in \mathbb{F}_{2}^{4}$, is obtained by blowing up a singular point $\vec{F}_{\vec{a}}$ in $T / \mathbb{Z}_{2}$. Hence for every lattice vector $\vec{\lambda} \in \Lambda$, the shift symmetry $\vec{x} \mapsto \vec{x}+\frac{1}{2} \vec{\lambda}$ for $\vec{x} \in \mathbb{R}^{4}$ induces a symmetry on $T / \mathbb{Z}_{2}$ which permutes the singular points by the corresponding shift on the hypercube $\mathbb{F}_{2}^{4}$. If $\lambda=\sum_{i=1}^{4} b_{i} \vec{\lambda}_{i}$ with generators $\vec{\lambda}_{1}, \ldots, \vec{\lambda}_{4}$ of $\Lambda$ as in (2.4) and $b_{i} \in\{0,1\}$ for all $i$, then this symmetry induces the holomorphic symplectic automorphism $t^{b}$ of the above proposition. This motivates our terminology of 
Definition 3.3.2 Consider a Kummer surface $X$ with underlying torus $T$, equipped with the induced dual Kähler class. We call the group $G_{t} \cong\left(\mathbb{Z}_{2}\right)^{4}$ of holomorphic symplectic automorphisms $t^{\vec{b}}, \vec{b} \in \mathbb{F}_{2}^{4}$, obtained in proposition 3.3.1 the TRANSLATIONAL AUTOMORPHISM GROUP OF $X$.

We now proceed to determine the structure of the symmetry group $G$ of a Kummer surface. First, the action of $G$ preserves the special structure of the homology of Kummer surfaces that we described in proposition 2.2.3, the statements of the following proposition follow immediately from the results of [22]:

Proposition 3.3.3 Consider a Kummer surface $X$ with underlying torus $T$, equipped with the induced dual Kähler class. Let $f$ denote a holomorphic symplectic automorphism of $X$ and $\alpha:=f_{*}$ its induced action on $H_{*}(X, \mathbb{Z})$.

With notations as in definition 2.2 .2 and proposition 2.2 .3 , for the Kummer lattice $\Pi$ we have $\alpha(\Pi)=\Pi$, and for $K=\pi_{*}\left(H_{2}(T, \mathbb{Z})\right)$ we have $\alpha(K)=K$.

There is an affine linear map $A$ on $\mathbb{F}_{2}^{4}$ such that the action of $\alpha$ on $\Pi$ is induced by $\alpha\left(E_{\vec{a}}\right)=$ $E_{A(\vec{a})}, \vec{a} \in \mathbb{F}_{2}^{4}$. In fact, A uniquely determines $f$.

Note that the translational automorphisms $t^{b}, \vec{b} \in \mathbb{F}_{2}^{4}$, found in proposition 3.3 .1 are precisely the holomorphic symplectic automorphisms of a Kummer surface which are given by translations $A(\vec{a})=\vec{a}+\vec{b}$ on the hypercube $\mathbb{F}_{2}^{4}$ according to proposition 3.3 .3 . We are now ready to describe the generic form of every symmetry group of a Kummer surface. As mentioned above, this description along with the ideas which lead to it are known to the experts. However, for the reader's convenience and since we have not found an appropriate reference in the literature, we recall its derivation:

Proposition 3.3.4 Consider a Kummer surface $X$ with underlying torus $T=T(\Lambda)$, equipped with the induced dual Kähler class. Let $G$ denote its symmetry group. Then the following holds:

1. The group $G$ is a semi-direct product $G_{t} \rtimes G_{T}$ with $G_{t}$ the translational automorphism group of definition 3.3 .2 and $G_{T}$ the group of those symplectic automorphisms of $X$ that are induced by the holomorphic symplectic automorphisms of $T$ that fix $0 \in \mathbb{C}^{2} / \Lambda=T$. In other word $11, G_{T} \cong G_{T}^{\prime} / \mathbb{Z}_{2}$ with $G_{T}^{\prime}$ the group of non-translational holomorphic symplectic automorphisms of $T$.

2. The group $G$ is isomorphic to a subgroup of $\left(\mathbb{Z}_{2}\right)^{4} \rtimes A_{7}$ with $A_{7}$ the group of even permutations on 7 elements.

Proof: Throughout this proof we use the notations introduced in definition 2.2 .2 and proposition 2.2 .3 .

1. Let $f \in G$ and $\alpha:=f_{*}$ as before. Proposition 3.3 .3 implies that $\alpha$ induces an automorphism of $K=\pi_{*}\left(H_{2}(T, \mathbb{Z})\right) \cong H_{2}(T, \mathbb{Z})(2)$ and thus an automorphism $\alpha_{T}^{\prime}$ of $H_{2}(T, \mathbb{Z})$. Then $\alpha_{T}^{\prime}$ acts as a Hodge isometry on $H_{2}(T, \mathbb{Z})$, since $\alpha$ does so on $H_{2}(X, \mathbb{Z})$ and complex structure and dual Kähler class of $X$ are induced by those on $T$. Hence by the global Torelli Theorem for complex tori, this means that $\alpha_{T}^{\prime}=\left(f_{T}^{\prime}\right)_{*}$ for some holomorphic symplectic automorphism $f_{T}^{\prime}$ of $T$. Let $f_{T} \in G_{T}$ denote the automorphism which $f_{T}^{\prime}$ induces on $X$ (see for example the proof of [22, Thm. 2]). We claim that

\footnotetext{
${ }^{11}$ The notion of holomorphic symplectic automorphisms on complex tori is defined completely analogously to our definition for K3 surfaces.
} 
$f=t^{\vec{b}} \circ f_{T}$ for some $\vec{b} \in \mathbb{F}_{2}^{4}$ and $t^{\vec{b}} \in G_{t}$ as in proposition 3.3.1. Indeed, by construction, $t_{*}:=\alpha \circ\left(\left(f_{T}\right)_{*}\right)^{-1}$ acts trivially on $K=\pi_{*}\left(H_{2}(T, \mathbb{Z})\right)$. Under gluing $\Pi$ to $K$ according to proposition 2.1.1. this trivial action must be compatible with the action of $t_{*}$ on $\Pi$. In particular, $t_{*}$ must induce an action on the hypercube $\mathbb{F}_{2}^{4}$ of labels $\vec{a}$ of the $E_{\vec{a}}$ which maps every affine plane in $\mathbb{F}_{2}^{4}$ to a parallel plane. One checks that this implies that $t_{*} \in G_{t}$.

The uniqueness of the decomposition $f=t^{\vec{b}} \circ f_{T}$ with $t^{\vec{b}} \in G_{t}$ and $f_{T} \in G_{T}$ and then $G \cong G_{t} \rtimes G_{T}$ now follow from proposition 3.3 .3 .

2. Since we already know that $G \cong G_{t} \rtimes G_{T}$ with $G_{t} \cong\left(\mathbb{Z}_{2}\right)^{4}$, it remains to show that $G_{T} \subset A_{7}$. However, this immediately follows from Fujiki's classification of holomorphic symplectic automorphism groups of complex tori [48, Lemma 3.1\&3.2]. Indeed, Fujiki proves that $G_{T}^{\prime}$ is isomorphic to a subgroup of one of the following groups: The cyclic groups $\mathbb{Z}_{4}$ or $\mathbb{Z}_{6}$ with $\mathbb{Z}_{4} / \mathbb{Z}_{2} \cong \mathbb{Z}_{2}$ and $\mathbb{Z}_{6} / \mathbb{Z}_{2} \cong \mathbb{Z}_{3}$, the binary dihedral groups $\mathcal{O}$ or $\mathcal{D}$ of orders 8 or 12 with $\mathcal{O} / \mathbb{Z}_{2} \cong \mathbb{Z}_{2} \times \mathbb{Z}_{2}$ and $\mathcal{D} / \mathbb{Z}_{2} \cong S_{3}$ (the permutation group on 3 elements), or the binary tetrahedral group $\mathcal{T}$ with $\mathcal{T} / \mathbb{Z}_{2} \cong A_{4}$ (the group of even permutations on 4 elements). Hence $G_{T}$ is isomorphic to a subgroup of $A_{6}$, the group of even permutations on 6 elements. With $A_{6} \subset A_{7}$ the claim follows.

Note that by our proof of proposition 3.3.4 every holomorphic symplectic automorphism group of a Kummer surface is in fact isomorphic to a subgroup of $\left(\mathbb{Z}_{2}\right)^{4} \rtimes A_{6}$. However, for reasons that will become clear later, we prefer working with the bigger group $\left(\mathbb{Z}_{2}\right)^{4} \rtimes A_{7}$. The proposition implies that the translational group $G_{t} \cong\left(\mathbb{Z}_{2}\right)^{4}$ is the symmetry group of generic Kummer surfaces with induced (dual) Kähler class.

Definition 3.3.5 We call the group $\left(\mathbb{Z}_{2}\right)^{4} \rtimes A_{7}$, where $A_{7}$ denotes the group of even permutations on 7 elements, the OVERARCHING FINITE SYMMETRY GROUP OF KUMMER SURFACES.

Let us now take a closer look at the lattices that are involved in the action of a symmetry group of a Kummer surface (see table 2 for a snapshot of lattices used in the following):

Proposition 3.3.6 Consider a Kummer surface $X$ with underlying torus $T$, equipped with the induced dual Kähler class. Let $G$ denote its symmetry group. Then the following holds, with notations as in proposition 2.2.4:

1. For the Kummer lattice $\Pi$ and the induced action of $G$ on it,

$$
\Pi^{G}=\Pi \cap H_{*}(X, \mathbb{Z})^{G}=\operatorname{span}_{\mathbb{Z}}\{e\} \quad \text { with } \quad e:=\frac{1}{2} \sum_{\vec{a} \in \mathbb{F}_{2}^{4}} E_{\vec{a}} .
$$

2. Let $L_{G}:=\left(H_{*}(X, \mathbb{Z})^{G}\right)^{\perp} \cap H_{*}(X, \mathbb{Z})$ as before and $v_{0} \in H_{0}(X, \mathbb{Z}), v \in H_{4}(X, \mathbb{Z})$ such that $\left\langle v_{0}, v\right\rangle=1$. With $G_{T}^{\prime}$ the group of holomorphic symplectic automorphisms of $T$ that fix $0 \in \mathbb{C}^{2} / \Lambda$, the lattice

$$
M_{G}:=L_{G} \oplus \operatorname{span}_{\mathbb{Z}}\left\{e, v_{0}-v\right\}
$$

is the orthogonal complement of the lattice $M_{G}^{\prime}:=\pi_{*}\left(H_{2}(T, \mathbb{Z})^{G_{T}^{\prime}}\right) \oplus \operatorname{span}_{\mathbb{Z}}\left\{v_{0}+v\right\}$ in $H_{*}(X, \mathbb{Z})$.

Proof: 
1. This follows immediately from the fact that $G$ contains the translational group $G_{t} \cong$ $\left(\mathbb{Z}_{2}\right)^{4}$ by proposition 3.3 .1 along with proposition 3.3.3.

2. From propositions 3.3 .3 and 3.3 .4 it follows that $H_{2}(X, \mathbb{Z})^{G}$ is obtained by gluing $\pi_{*}\left(H_{2}(T, \mathbb{Z})^{G_{T}^{\prime}}\right)$ and $\Pi^{G}$ according to proposition 2.1.1. Hence the claim follows from $H_{*}(X, \mathbb{Z})^{G}=H_{2}(X, \mathbb{Z})^{G} \oplus \operatorname{span}_{\mathbb{Z}}\left\{v_{0}, v\right\}$ together with $\Pi^{G}=\operatorname{span}_{\mathbb{Z}}\{e\}$ along with proposition 2.1.1.

We are now ready to prove and appreciate the following result, which is crucial to our investigations:

Theorem 3.3.7 Let $X$ denote a Kummer surface with underlying torus $T$, equipped with the complex structure and dual Kähler class which are induced from $T$. Let $G$ denote the symmetry group of $X$. With notations as in proposition 3.3.6, in particular $L_{G}=\left(H_{*}(X, \mathbb{Z})^{G}\right)^{\perp} \cap$ $H_{*}(X, \mathbb{Z})$ and $v_{0} \in H_{0}(X, \mathbb{Z}), v \in H_{4}(X, \mathbb{Z})$ such that $\left\langle v_{0}, v\right\rangle=1$, and $e:=\frac{1}{2} \sum_{\vec{a} \in \mathbb{F}_{2}^{4}} E_{\vec{a}}$, the lattice $M_{G}(-1)$ with

$$
M_{G}:=L_{G} \oplus \operatorname{span}_{\mathbb{Z}}\left\{e, v_{0}-v\right\}
$$

can be primitively embedded in the Niemeier lattice $N$ of type $A_{1}^{24}$.

Proof: We apply Nikulin's theorem 2.1 .3 to the lattice $\Lambda:=M_{G}(-1)$ of signature $\left(\ell_{+}, \ell_{-}\right)$ and to the pair of integers $\left(\gamma_{+}, \gamma_{-}\right)=(24,0)$. Throughout the proof we use the notations introduced in definition 2.2.2, proposition 2.2 .3 and proposition 3.3 .6 .

Notice first that by proposition 3.3 .6 our lattice $M_{G}$ contains the lattice

$$
\begin{aligned}
\widehat{M}_{G}:=\widehat{K}_{G} \oplus \Pi \oplus \operatorname{span}_{\mathbb{Z}}\left\{v_{0}-v\right\} \\
\text { with } \widehat{K}_{G}:=\pi_{*}\left(\left(H_{2}(T, \mathbb{Z})^{G_{T}^{\prime}}\right)^{\perp} \cap H_{2}(T, \mathbb{Z})\right) .
\end{aligned}
$$

In fact property 2. in proposition 3.3.6 implies $\operatorname{rk}\left(M_{G}\right)=\operatorname{rk}\left(\widehat{M}_{G}\right)$. Hence the lattice $M_{G}(-1)$ has signature $\left(l_{+}, l_{-}\right)=(17+k, 0)$, where $17=\operatorname{rk}\left(\Pi \oplus \operatorname{span}_{\mathbb{Z}}\left\{v_{0}-v\right\}\right)$ and $k:=\operatorname{rk}\left(\widehat{K}_{G}\right)$. In particular, we have $k \leq 3$, since $H_{2}(T, \mathbb{R})^{G_{T}^{\prime}}=H_{2}(T, \mathbb{Z})^{G_{T}^{\prime}} \otimes \mathbb{R}$ contains the positive definite 3-dimensional subspace $\Sigma_{T} \subset H_{2}(T, \mathbb{R})$ yielding complex structure and dual Kähler class of $T$, such that $H_{2}(T, \mathbb{Z})^{G_{T}^{\prime}}$ has at least rank 3. Hence condition 1. in theorem 2.1.3 holds.

By condition 2. in proposition 3.3.6, $M_{G}$ is the orthogonal complement of the primitive sublattice $M_{G}^{\prime}=\pi_{*}\left(H_{2}(T, \mathbb{Z})^{G_{T}^{\prime}}\right) \oplus \operatorname{span}_{\mathbb{Z}}\left\{v_{0}+v\right\}$ in the unimodular lattice $H_{*}(X, \mathbb{Z})$. Hence in particular $A_{q}=\left(M_{G}\right)^{*} / M_{G} \cong\left(M_{G}^{\prime}\right)^{*} / M_{G}^{\prime}$ and

$$
\ell\left(A_{q}\right)=\ell\left(\left(M_{G}^{\prime}\right)^{*} / M_{G}^{\prime}\right) \leq \operatorname{rk}\left(M_{G}^{\prime}\right)=7-k=\gamma_{+}+\gamma_{-}-l_{+}-l_{-},
$$

proving condition 2. of theorem 2.1.3.

Since $M_{G}(-1)$ contains the lattice $\operatorname{span}_{\mathbb{Z}}\left\{v_{0}-v\right\}(-1)$ of type $A_{1}$ as direct summand, condition 3.i. of Nikulin's theorem is also immediate.

It hence remains to be shown that

$$
\ell\left(A_{q_{p}}\right)<7-k=\gamma_{+}+\gamma_{-}-l_{+}-l_{-} \text {for every prime } p \neq 2 .
$$

Using $\widehat{M}_{G} \subset M_{G} \subset M_{G}^{*} \subset \widehat{M}_{G}^{*}$, we find $M_{G}^{*} / M_{G} \cong\left(M_{G}^{*} / \widehat{M}_{G}\right) /\left(M_{G} / \widehat{M}_{G}\right)$ and therefore

$$
A_{q}=M_{G}^{*} / M_{G} \subset\left(\widehat{M}_{G}^{*} / \widehat{M}_{G}\right) /\left(M_{G} / \widehat{M}_{G}\right) .
$$


Since $\widehat{M}_{G}^{*} / \widehat{M}_{G}=\widehat{K}_{G}^{*} / \widehat{K}_{G} \times\left(\mathbb{Z}_{2}\right)^{7}$ with $\ell\left(\widehat{K}_{G}^{*} / \widehat{K}_{G}\right) \leq \operatorname{rk}\left(\widehat{K}_{G}\right) \leq 3$, for every prime $p \neq 2$ this implies

$$
\ell\left(A_{q_{p}}\right) \leq \operatorname{rk}\left(\widehat{K}_{G}\right) \leq 3<7-k=\gamma_{+}+\gamma_{-}-l_{+}-l_{-} .
$$

Equation (3.7) is proved, so the assumptions of Nikulin's theorem 2.1.3 hold, which implies that $M_{G}(-1)$ can be primitively embedded into some Niemeier lattice $\widetilde{N}$. By the above, $\Pi(-1) \subset \widehat{M}_{G}(-1) \subset M_{G}(-1)$ and thus $\widetilde{N} \cong N$ by proposition 2.3.3.

To fully appreciate the theorem, note that it immediately implies the following

Corollary 3.3.8 Let $X$ denote a Kummer surface with underlying torus $T$, equipped with the induced dual Kähler class. Let $G$ denote the symmetry group of $X$. Then in Kondo's proof of Mukai's theorem 3.2.5, one can use the Niemeier lattice $N$ of type $A_{1}^{24}$. Moreover, there is an isometry $i_{G}$ of a primitive sublattice $M_{G}$ of $H_{*}(X, \mathbb{Z})$ to a primitive sublattice of $N(-1)$ which is equivariant with respect to the natural actions of $G$, where $M_{G}$ contains the lattice $L_{G}=\left(H_{*}(X, \mathbb{Z})^{G}\right)^{\perp} \cap H_{*}(X, \mathbb{Z})$, the Kummer lattice $\Pi$ of $X$, and the vector $v_{0}-v$. Here, $G$-equivariance of $i_{G}$ means that $i_{G}\left(g_{*}(v)\right)=g_{*}\left(i_{G}(v)\right)$ for every $g \in G$ and $v \in M_{G}$.

This means that our theorem 3.3.7 offers an improvement of Kondo's techniques for all Kummer surfaces with induced dual Kähler class, since our lattice $M_{G}$ contains the sublattice $L_{G}$ which Kondo identifies in his Niemeier lattices and has rank rk $\left(M_{G}\right)=\operatorname{rk}\left(L_{G}\right)+2$.

Recall that by means of the Kummer construction, we are using a fixed marking for all our Kummer surfaces, as was explained at the end of section 2.2. In particular, we may smoothly vary the generators $\vec{\lambda}_{1}, \ldots, \vec{\lambda}_{4}$ of the underlying lattice $\Lambda \subset \mathbb{C}^{2}$ of $T(\Lambda)$ and thus vary between distinct Kummer surfaces $T \widetilde{(\Lambda) / \mathbb{Z}_{2}}$. This amounts to a variation along a Kummer path in the smooth connected cover $\widetilde{\mathcal{M}}_{h k}$ of the moduli space of hyperkähler structures, as was explained at the end of section 3.1. Along a generic such path, both the symmetry group $G$ and the lattice $M_{G}$ change, but as $G$ varies all the lattices $M_{G}$ share the same sublattice $\Pi \oplus \operatorname{span}_{\mathbb{Z}}\left\{v_{0}-v\right\}$. Since according to proposition 2.3.5 the Kummer lattice $\Pi$ allows a unique primitive embedding $\Pi \hookrightarrow N(-1)$ up to automorphisms of $N$, we can always find an embedding $i_{G}: M_{G} \hookrightarrow N(-1)$ as in theorem 3.3.7 such that $\left(i_{G}\right)_{\mid \Pi}$ is induced by $E_{\vec{a}} \mapsto f_{I^{-1}(\vec{a})}$ for all $\vec{a} \in \mathbb{F}_{2}^{4}$ with $I$ as in (2.14). For brevity, we say that we choose $i_{G}$ WITH CONSTANT $i_{G}(\Pi)=\widetilde{\Pi}$ ALONG KUMmer PATHS IN $\widetilde{\mathcal{M}}_{h k}$. Let us briefly discuss some properties of $i_{G}(\Pi) \hookrightarrow M_{G} \hookrightarrow N(-1)$. To this end we remark that the only automorphisms $\gamma \in \operatorname{Aut}(N)$ which act as the identity on the sublattice $\widetilde{\Pi} \subset N$ in (2.11) are compositions of sign flips $f_{n} \mapsto-f_{n}$ with $n \in \mathcal{O}_{9}$. Indeed, without loss of generality assume that $\gamma$ acts as permutation on $\left\{f_{1}, \ldots, f_{24}\right\}$. Then $\gamma_{\mid \widetilde{\Pi}}=\mathrm{id}_{\widetilde{\Pi}}$ implies that $\gamma$ is induced by an element of the maximal subgroup $\left(\mathbb{Z}_{2}\right)^{4} \rtimes A_{8}$ of $M_{24}$ which stabilizes the octad $\mathcal{O}_{9}$. This group acts as $\operatorname{Aff}\left(\mathbb{F}_{2}^{4}\right)=$ $\left(\mathbb{Z}_{2}\right)^{4} \rtimes \mathrm{GL}_{4}\left(\mathbb{F}_{2}\right) \cong\left(\mathbb{Z}_{2}\right)^{4} \rtimes A_{8}$ on the hypercube $\mathbb{F}_{2}^{4}$ underlying the complement octad of $\mathcal{O}_{9}$ (see [29, Thm. 2.10]), thus in particular its induced action on $\widetilde{\Pi}$ is faithful. Hence $\gamma_{\mid \widetilde{\Pi}}=\mathrm{id}_{\widetilde{\Pi}}$ implies $\gamma=\mathrm{id}$. As a consequence, we cannot expect $i_{G}\left(\Pi \oplus \operatorname{span}_{\mathbb{Z}}\left\{v_{0}-v\right\}\right) \subset N(-1)$ to be constant along Kummer paths in $\widetilde{\mathcal{M}}_{h k}$. In other words, $i_{G}\left(v_{0}-v\right)$ cannot be chosen freely among the roots $\pm f_{n}$ with $n \in \mathcal{O}_{9}$. We will come back to this observation in the next section and in the conclusions.

\section{The overarching finite symmetry group of Kummer surfaces}

By Mukai's theorem 3.2.5, certain comparatively small subgroups $G$ of the Mathieu group $M_{24}$ occur as holomorphic symplectic automorphism groups of K3 surfaces $X$. The correlation with $M_{24}$ is made more explicit by Kondo's proof of this theorem, by which for the smallest 
primitive sublattice $L_{G} \subset H_{*}(X, \mathbb{Z})$ with the property that $G$ acts trivially on $\left(L_{G}\right)^{\perp}$ there also exists a primitive embedding of $L_{G}$ into some Niemeier lattice $\widetilde{N}(-1)$, such that the embedding is equivariant with respect to a natural action of $G$ on $\widetilde{N}$. Restricting our attention to Kummer surfaces with their induced dual Kähler classes, our theorem 3.3.7 allows us to replace the lattice $L_{G}$ by a lattice $M_{G}$ of higher rank (namely, $\operatorname{rk}\left(M_{G}\right)=\operatorname{rk}\left(L_{G}\right)+2$ ), and to replace the Niemeier lattice $\widetilde{N}$, which is not further specified by Kondo's construction, by the Niemeier lattice $N$ of type $A_{1}^{24}$. In other words, a part of the K3-homology can be isometrically identified with a sublattice of the Niemeier lattice $N(-1)$, where the group $M_{24}$, whose role we would like to understand better, acts naturally (see proposition 2.3.2). This section is devoted to developing a technique by which we extend this identification even further, namely to a linear bijection between the two lattices $H_{*}(X, \mathbb{Z})$ and $N(-1)$. This bijection identifies two different primitive sublattices $M_{\mathcal{T}_{192}}$ and $M_{\mathcal{T}_{64}}$ of rank 20 in $H_{*}(X, \mathbb{Z})$, corresponding to two distinct Kummer surfaces, isometrically with their images. Moreover, these embeddings are equivariant with respect to the actions of the respective symmetry groups $\mathcal{T}_{192}$ on $M_{\mathcal{T}_{192}}$ and $\mathcal{T}_{64}$ on $M_{\mathcal{T}_{64}}$, which allows us to combine these groups to a bigger one, yielding the overarching finite symmetry group of Kummer surfaces (see definition 3.3.5). This group is by orders of magnitude larger than the largest holomorphic symplectic automorphism group of any K3 surface. In fact, we find a Kummer path in $\widetilde{\mathcal{M}}_{h k}$ with constant $i_{G}(\Pi)=\widetilde{\Pi}$ along it, which connects the two above mentioned Kummer surfaces smoothly and such that $\Theta$ restricts to a $G$-equivariant isometry $i_{G}: M_{G} \hookrightarrow N(-1)$ for each Kummer surface along the path.

\subsection{The translational automorphism group $G_{t} \cong\left(\mathbb{Z}_{2}\right)^{4}$}

As a first step of our construction, we consider the translational automorphism group $G_{t}$ of definition 3.3 .2 , which all Kummer surfaces share. We proceed analogously to Kondo's proof of Mukai's theorem 3.2.5.

If $G=G_{t}$ is the symmetry group of a Kummer surface $X$ with underlying torus $T$, then with notations as in proposition 2.2.3 and theorem 3.3.7, in particular $K=\pi_{*}\left(H_{2}(T, \mathbb{Z})\right)$, we have

$$
H_{*}(X, \mathbb{Z})^{G}=H_{0}(X, \mathbb{Z}) \oplus K \oplus \operatorname{span}_{\mathbb{Z}}\{e\} \oplus H_{4}(X, \mathbb{Z})
$$

and thus $M_{G}=\Pi \oplus \operatorname{span}_{\mathbb{Z}}\left\{v_{0}-v\right\}$, which can be primitively embedded in $N(-1)$ by theorem 3.3.7. In fact, by proposition 2.2.4, we have $M_{G}=\mathcal{P}$, where we know how to glue $H_{*}(X, \mathbb{Z})$ from the lattices $\mathcal{P}$ and $\mathcal{K}=\mathcal{P}^{\perp} \cap H_{*}(X, \mathbb{Z})$, and (2.19) shows $\mathcal{P} \cong \widetilde{\mathcal{P}}_{n_{0}}(-1)$ for a primitive sublattice $\widetilde{\mathcal{P}}_{n_{0}}$ of $N, n_{0} \in \mathcal{O}_{9}$ arbitrary, along with the relevant gluing prescription for $N$. By the proof of proposition 2.3 .4 , an isometry $\mathcal{P} \longrightarrow \widetilde{\mathcal{P}}_{n_{0}}(-1)$ can be induced by $E_{\vec{a}} \mapsto f_{I^{-1}(\vec{a})}$ for every $\vec{a} \in \mathbb{F}_{2}^{4}$, with $I$ as in (2.14), along with $v_{0}-v \mapsto f_{n_{0}}$. Since $G_{t}$ acts on $\mathcal{P}$ by automorphisms, we obtain an induced action of $G_{t} \cong\left(\mathbb{Z}_{2}\right)^{4}$ on $\widetilde{\mathcal{P}}_{n_{0}}$ by enforcing our isometry $\mathcal{P} \longrightarrow \widetilde{\mathcal{P}}_{n_{0}}(-1)$ to be $G_{t}$-equivariant. By construction, $G_{t}$ acts trivially on $\mathcal{P}^{*} / \mathcal{P} \cong \widetilde{\mathcal{P}}_{n_{0}}^{*} / \widetilde{\mathcal{P}}_{n_{0}}$, and thus the action can be extended to one on $N$ acting trivially on the orthogonal complement of $\widetilde{\mathcal{P}}_{n_{0}}$ (see [11, Prop. 1.1]). The construction yields the following

Proposition 4.1.1 Consider a Kummer surface $X$ with underlying torus $T$, equipped with the induced dual Kähler class. Let $G$ denote the symmetry group of $X$ and $G_{t} \subset G$ the translational automorphism group according to proposition 3.3.1.

There exists a primitive embedding $i_{G}: M_{G} \hookrightarrow N(-1)$ of the lattice $M_{G} \subset H_{*}(X, \mathbb{Z})$ defined in theorem 3.3 .7 into the Niemeier lattice $N(-1)$ of type $A_{1}^{24}$ with the following properties: Consider the lattices $\mathcal{P}$ and $\widetilde{\mathcal{P}}_{n_{0}}$ with the notations of propositions 2.2.4 and 2.3.6, where $\mathcal{P} \subset M_{G}$ by corollary 3.3.8. Then

$$
i_{G}: E_{\vec{a}} \longmapsto f_{I^{-1}(\vec{a})} \quad \text { for every } \quad \vec{a} \in \mathbb{F}_{2}^{4},
$$


where $I$ is the map (2.14), and $i_{G}(\mathcal{P})=\widetilde{\mathcal{P}}_{n_{0}}(-1)$ for some $n_{0} \in \mathcal{O}_{9}$.

Moreover, by means of the embedding $i_{G}: M_{G} \hookrightarrow N(-1)$ the action of the translational automorphism group $G_{t}$ on $X$ induces an action of $\left(\mathbb{Z}_{2}\right)^{4} \subset M_{24}$ on $N$ which is generated by the following four involutions:

$$
\begin{aligned}
& \iota_{1}:=(1,11)(2,22)(4,20)(7,12)(8,17)(10,18)(13,21)(14,16), \\
& \iota_{2}:=(1,13)(2,12)(4,14)(7,22)(8,10)(11,21)(16,20)(17,18), \\
& \iota_{3}:=(1,14)(2,17)(4,13)(7,10)(8,22)(11,16)(12,18)(20,21), \\
& \iota_{4}:=(1,17)(2,14)(4,12)(7,20)(8,11)(10,21)(13,18)(16,22),
\end{aligned}
$$

where as in proposition 2.3 .2 the elements of $M_{24}$ are viewed as permutations in $S_{24}$ whose action on $N$ is induced by a permutation of the $f_{1}, \ldots, f_{24}$.

Proof: With notations as above, theorem 3.3.7 implies the existence of a primitive embedding $i: M_{G} \hookrightarrow N(-1)$. In particular, we find a primitive sublattice $\widehat{\mathcal{P}}(-1) \subset i\left(M_{G}\right)$ such that $\widehat{\mathcal{P}}$ is isometric to any $\widetilde{\mathcal{P}}_{n_{0}}$ with $n_{0} \in \mathcal{O}_{9}$. By proposition 2.3.6 we have $\widetilde{\mathcal{P}}_{n_{0}}=\widetilde{\Pi} \oplus \operatorname{span}_{\mathbb{Z}}\left\{f_{n_{0}}\right\}$ with $\widetilde{\Pi} \cong \Pi(-1)$ for the Kummer lattice $\Pi$ of (2.5). Since the $\pm f_{n}$ are the only vectors in $N$ on which the quadratic form takes value 2 by (2.9), this implies $\widehat{\mathcal{P}}=\widehat{\Pi} \oplus \operatorname{span}_{\mathbb{Z}}\left\{f_{\widehat{n}_{0}}\right\}$ with $\widehat{\Pi} \cong \Pi(-1)$ and for some $\widehat{n}_{0} \in \mathcal{I}$. Our uniqueness result in proposition 2.3.5 implies that there is a lattice automorphism $\alpha$ of $N$ with $\alpha(\widehat{\Pi})=\widetilde{\Pi}$ and $\alpha\left(i\left(E_{I(n)}\right)\right)=f_{n}$ for every $n \in \mathcal{I} \backslash \mathcal{O}_{9}$. In other words, $\alpha \circ i$ gives a primitive embedding of $M_{G}$ in $N(-1)$ which embeds the lattice $\Pi$ as claimed. Moreover, $\alpha \circ i\left(v_{0}-v\right)= \pm \alpha\left(f_{\widehat{n}_{0}}\right)= \pm f_{n_{0}}$ for some $n_{0} \in \mathcal{O}_{9}$ due to (2.9) and $v_{0}-v \in \Pi^{\perp}$. Hence $\alpha \circ i$ or its composition with the automorphism of $N$ induced by $f_{n_{0}} \mapsto-f_{n_{0}}$ (see proposition 2.3.2) yields an embedding $i_{G}: M_{G} \hookrightarrow N(-1)$ with the properties claimed.

We now apply the methods described before the statement of our proposition to induce an action of $G_{t} \cong\left(\mathbb{Z}_{2}\right)^{4}$ on $N$ with respect to which the embedding $i_{G}$ is equivariant. In other words, with $\vec{b}_{1}, \ldots, \vec{b}_{4}$ the standard basis of $\mathbb{F}_{2}^{4}$, for each $k \in\{1, \ldots, 4\}$ we impose $I\left(\iota_{k}(n)\right)=I(n)+\vec{b}_{k}$ for all $n \in \mathcal{I} \backslash \mathcal{O}_{9}$, where $I$ is the map (2.14). This uniquely determines the permutations $\iota_{1}, \ldots, \iota_{4}$ of (4.1), which as one confirms as a cross-check 12 are elements of $M_{24}$.

The precise form of the permutations in (4.1) depends on our specific choice of primitive embedding for the Kummer lattice $\Pi$ in $N(-1)$, described in proposition 2.3.4 and in its proof. Indeed, the image $\widetilde{\Pi}(-1)$ of $\Pi$ under the primitive embedding solely depends on the choice of the special octad $\mathcal{O}_{9}$ used in (2.11). The isometry from $\Pi$ to $\widetilde{\Pi}(-1)$ induced by this embedding then solely depends on the choice of the octads $\mathcal{H}_{1}, \ldots, \mathcal{H}_{5}$ in (2.12). As explained in the discussion of proposition 2.3.4, the subspace of the Golay code generated by $\mathcal{H}_{1}, \ldots, \mathcal{H}_{4}$ is dual to the hypercube $\mathbb{F}_{2}^{4}$ built on $\mathcal{I} \backslash \mathcal{O}_{9}$ by means of our map $I$. Then the choice of $\mathcal{H}_{1}, \ldots, \mathcal{H}_{5}$ amounts to the choice of an affine basis of the underlying affine 4-dimensional space.

Note that in general, the symmetry group $G$ of a Kummer surface contains the translational automorphism group $G_{t}$ as a proper subgroup. The primitive embedding of $M_{G}$ in $N(-1)$ found in proposition 4.1.1, where $\widetilde{\mathcal{P}}_{n_{0}}(-1)$ is a primitive sublattice of the image of $M_{G}$, can then be used to induce a faithful $G$-action on the Niemeier lattice $N$ by enforcing the embedding $i_{G}: M_{G} \hookrightarrow N(-1)$ to be $G$-equivariant, by the very same ideas that lead to proposition 4.1 .1 .

We claim that the embedding $i_{G}: M_{G} \hookrightarrow N(-1)$ can be extended to a linear bijection $\theta$ between the K3-homology $H_{*}(X, \mathbb{Z})$ and $N(-1)$. Indeed, $H_{*}(X, \mathbb{Z})$ can be glued from $\mathcal{P}$ and

\footnotetext{
${ }^{12}$ See appendix $\mathrm{A}$ for a definition of the extended binary Golay code $\mathcal{G}_{24}$ and for the description of a technique that may be used to prove that a given permutation of $\mathcal{I}$ preserves $\mathcal{G}_{24}$, for instance by verifying that its action on the Golay code basis A.2 yields Golay codewords.
} 
its orthogonal complement $\mathcal{K}$, while $N$ can be glued from $\widetilde{\mathcal{P}}_{n_{0}}$ and its orthogonal complement $\widetilde{\mathcal{K}}_{n_{0}}$, by means of propositions 2.2 .4 and 2.3.6. respectively. Since $\mathcal{P}$ is already isometrically identified with $\widetilde{\mathcal{P}}_{n_{0}}(-1)$, the extension to a bijection $\theta: H_{*}(X, \mathbb{Z}) \longrightarrow N(-1)$ amounts to the construction of a bijection $\mathcal{K} \rightarrow \widetilde{\mathcal{K}}_{n_{0}}(-1)$ which induces an isometry from $M_{G} \cap \mathcal{K}$ to its image, and which is compatible with gluing. The latter in turn amounts to compatibility with the isometries (up to signature inversion) $g: \mathcal{K}^{*} / \mathcal{K} \longrightarrow \mathcal{P}^{*} / \mathcal{P}$ and $\widetilde{g}_{n_{0}}: \widetilde{\mathcal{K}}_{n_{0}}^{*} / \widetilde{\mathcal{K}}_{n_{0}} \longrightarrow \widetilde{\mathcal{P}}_{n_{0}}^{*} / \widetilde{\mathcal{P}}_{n_{0}}$ which are so fundamental to the gluing procedure, and which in particular, by means of $\mathcal{P} \cong \widetilde{\mathcal{P}}_{n_{0}}(-1)$, yield an isometry $\mathcal{K}^{*} / \mathcal{K} \longrightarrow \widetilde{\mathcal{K}}_{n_{0}}^{*} / \widetilde{\mathcal{K}}_{n_{0}}$. In other words, we need to find a lift of $\mathcal{K}^{*} / \mathcal{K} \longrightarrow \widetilde{\mathcal{K}}_{n_{0}}^{*} / \widetilde{\mathcal{K}}_{n_{0}}$ to a bijection $\mathcal{K}^{*} \rightarrow \widetilde{\mathcal{K}}_{n_{0}}^{*}(-1)$ which induces a $G$-equivariant isometry from $M_{G} \cap \mathcal{K}$ to its image. This is always possible, and our compatibility conditions severely restrict the number of choices. Indeed, by construction $M_{G} \cap \mathcal{K}$ can be isometrically identified with $\left(\widetilde{M}_{G} \cap \widetilde{\mathcal{K}}_{n_{0}}\right)(-1)$, where $\widetilde{M}_{G}$ denotes the image of $M_{G}$ under its primitive embedding into $N$. This determines the lift on a sublattice whose rank $7-r_{G}$ depends on the group $G$. Hence we only need to extend the isometry $M_{G} \cap \mathcal{K} \longrightarrow\left(\widetilde{M}_{G} \cap \widetilde{\mathcal{K}}_{n_{0}}\right)(-1)$ to a lift of $\mathcal{K}^{*} / \mathcal{K} \longrightarrow \widetilde{\mathcal{K}}_{n_{0}}^{*} / \widetilde{\mathcal{K}}_{n_{0}}$. Since $\mathcal{K}^{*} / \mathcal{K} \cong\left(\mathbb{Z}_{2}\right)^{7}$ according to proposition 2.2.4. and thus $\mathcal{K}^{*} / \mathcal{K}$ can be generated by no less than 7 elements, while the lattice $\mathcal{K}$ has rank 7 as well, we are certain to find $r_{G}=\operatorname{rk}\left(\left(M_{G}\right)^{\perp} \cap \mathcal{K}\right)$ compatibility conditions for the lift to $\left(M_{G}\right)^{\perp} \cap \mathcal{K}$. Since the translational group $G_{t} \cong\left(\mathbb{Z}_{2}\right)^{4}$ is the symmetry group of a generic Kummer surface, any bijection $\theta$ as above is compatible with the generic symmetry group of Kummer surfaces.

In our construction we work with the tetrahedral Kummer surface where $G=\mathcal{T}_{192}$, a group of order 192 (see section 4.2), and with the Kummer surface associated to the square torus where $G=\mathcal{T}_{64}$, a group of order 64 (see section 4.3). In both cases, the lattice $\left(M_{G}\right)^{\perp} \cap \mathcal{K}$, on which our lift is not uniquely determined up to lattice automorphisms by imposing that it induces $i_{G}: M_{G} \hookrightarrow N(-1)$, has the minimal possible rank $r_{G}=4$.

Let us discuss the compatibility conditions for our bijection $\theta: H_{*}(X, \mathbb{Z}) \longrightarrow N(-1)$ which already arise from the construction so far, imposing equivariance only with respect to the translational automorphism group $G_{t}$. In other words, let us discuss possible lifts of $\mathcal{K}^{*} / \mathcal{K} \longrightarrow$ $\widetilde{\mathcal{K}}_{n_{0}}^{*} / \widetilde{\mathcal{K}}_{n_{0}}$ to $\mathcal{K}^{*}$. Recall from proposition 2.2 .4 that $\mathcal{K}^{*} / \mathcal{K}$ is generated by the $\frac{1}{2} \pi_{*} \lambda_{i j}$ along with $\overline{\frac{1}{2}\left(v_{0}+v\right)}$ which under the composition of $g: \mathcal{K}^{*} / \mathcal{K} \longrightarrow \mathcal{P}^{*} / \mathcal{P}$ with the map induced by our isometry $\mathcal{P} \cong \widetilde{\mathcal{P}}_{n_{0}}(-1)$ are mapped to the $\overline{\frac{1}{2} \sum_{n \in \widetilde{P}_{i j} f_{n}}}$ and $\overline{\frac{1}{2} f_{n_{0}}}$, respectively. Here, $\widetilde{P}_{i j}$ is the quadruplet of labels in $\mathcal{I}$ which under the map $I$ corresponds to the plane $P_{i j} \subset \mathbb{F}_{2}^{4}$ in (2.7). Taking preimages under the map $\widetilde{g}_{n_{0}}: \widetilde{\mathcal{K}}_{n_{0}}^{*} / \widetilde{\mathcal{K}}_{n_{0}} \longrightarrow \widetilde{\mathcal{P}}_{n_{0}}^{*} / \widetilde{\mathcal{P}}_{n_{0}}$ of proposition 2.3.6, we find that $\mathcal{K}^{*} / \mathcal{K} \longrightarrow \widetilde{\mathcal{K}}_{n_{0}}^{*} / \widetilde{\mathcal{K}}_{n_{0}}$ is given by

$$
\overline{\frac{1}{2} \pi_{*} \lambda_{i j}} \mapsto \overline{q_{i j}} \text { for } i j=12,34,13,24,14,23, \quad \overline{\frac{1}{2}\left(v_{0}+v\right)} \mapsto \overline{\frac{1}{2} \sum_{n \in \mathcal{O}_{9} \backslash\left\{n_{0}\right\}} f_{n}},
$$

where the $\overline{q_{i j}}$ can be represented, for example, by the $q_{i j}$ of (2.15). We impose the additional constraint that the $\frac{1}{2} \pi_{*} \lambda_{i j}$ and $\frac{1}{2}\left(v+v_{0}\right)$ should map to representatives of elements in $\widetilde{\mathcal{K}}_{n_{0}}^{*} / \widetilde{\mathcal{K}}_{n_{0}}$ of minimal length under our lift. This is an aesthetic choice which is not required mathematically. If this constraint is not imposed, then in equation (4.2) we have to add terms of the form $2 \Delta_{i j}$ with $\Delta_{i j} \in \widetilde{\mathcal{K}}_{n_{0}}$ on the right hand side. We will see that in the examples studied in this work, it is possible to impose $\Delta_{i j}=0$ for all $i, j$, and then lifting to a bijection $\mathcal{K}^{*} \rightarrow \widetilde{\mathcal{K}}_{n_{0}}^{*}$ amounts to implementing

$$
K \ni \pi_{*} \lambda_{i j} \mapsto \sum_{n \in Q_{i j}}\left( \pm f_{n}\right) \in \widetilde{K}, \quad v_{0}+v \mapsto \sum_{n \in \mathcal{O}_{9} \backslash\left\{n_{0}\right\}}\left( \pm f_{n}\right)
$$

where $\overline{\frac{1}{2} \sum_{n \in Q_{i j}}\left( \pm f_{n}\right)}=\overline{q_{i j}} \in \widetilde{K}^{*} / \widetilde{K}$ and thus $\frac{1}{2} \sum_{n \in Q_{i j}}\left( \pm f_{n}\right)$ can be glued to $\frac{1}{2} \sum_{n \in \widetilde{P}_{i j}} f_{n}$ in $N$. In other words, $Q_{i j} \subset \mathcal{O}_{9}$ must be a quadruplet of labels such that $Q_{i j} \cup \widetilde{P}_{i j}$ gives an 
octad in the Golay code $\mathcal{G}_{24}$. In fact, each such quadruplet $Q_{i j}$ must form an octad of the Golay code with every quadruplet of labels which under $I$ corresponds to a hypercube plane parallel to $P_{i j}$. This turns out to leave a choice of two complementary quadruplets in $\mathcal{O}_{9}$ for each label $i j$ :

$$
\begin{array}{llll}
Q_{12}=\{3,6,15,19\} & \text { or } & \{5,9,23,24\}, \\
Q_{13}=\{6,15,23,24\} & \text { or } & \{3,5,9,19\}, \\
Q_{14}=\{3,9,15,24\} & \text { or } & \{5,6,19,23\}, \\
Q_{23}=\{3,9,15,23\} & \text { or } & \{5,6,19,24\}, \\
Q_{24}=\{15,19,23,24\} & \text { or } & \{3,5,6,9\}, \\
Q_{34}=\{6,9,15,19\} & \text { or } & \{3,5,23,24\},
\end{array}
$$

where

$$
K^{*} / K \stackrel{\cong}{\longrightarrow} \widetilde{K}^{*} / \widetilde{K}, \quad \overline{\frac{1}{2} \pi_{*} \lambda_{i j}} \mapsto \overline{\frac{1}{2} \sum_{n \in Q_{i j}} f_{n}} .
$$

Note that in (2.15) we have chosen the first quadruplet listed in (4.3) for the $Q_{i j}$, throughout. In the following subsections, we show that with this choice, surprisingly, the resulting bijection $\Theta$ induces isometric embeddings of the lattices $M_{G}$ for two distinct Kummer surfaces, the tetrahedral Kummer surface $X_{D_{4}}$ with $G=\mathcal{T}_{192}$ and the Kummer surface $X_{0}$ of the square torus with $G=\mathcal{T}_{64}$. Our choice of the $Q_{i j}$, up to a few choices of signs, is in fact unique with the property that for both groups $G$, the bijection $\Theta$ induces a $G$-equivariant isometric embedding of $M_{G}$ in $N(-1)$.

\subsection{The tetrahedral Kummer surface}

By the above, we are looking for a linear bijection $\Theta: H_{*}(X, \mathbb{Z}) \longrightarrow N(-1)$ which induces an isometry between as large sublattices of $H_{*}(X, \mathbb{Z})$ and of $N(-1)$ as possible. By theorem 3.3.7. for a Kummer surface $X$ with its induced dual Kähler class and with symmetry group $G$, we already know that a primitive sublattice $M_{G}$ of $H_{*}(X, \mathbb{Z})$, with $\operatorname{rk}\left(M_{G}\right)=\operatorname{rk}\left(L_{G}\right)+2$ for the lattice $L_{G}$ found by Kondo, can be primitively embedded in $N(-1)$. Note that $\operatorname{rk}\left(M_{G_{1}}\right) \geq$ $\operatorname{rk}\left(M_{G_{2}}\right)$ if $G_{1} \supseteq G_{2}$. Our construction therefore sets out from the study of the Kummer surface whose holomorphic symplectic automorphism group has maximal order among all Kummer surfaces with their induced dual Kähler classes. According to proposition 3.3.4, this amounts to a Kummer surface whose underlying torus $T=T(\Lambda)$ has the largest group $G_{T}^{\prime}$ of non-translational holomorphic symplectic automorphisms. By Fujiki's results [48, Lemma $3.1 \& 3.2]$, this is the torus $T\left(\Lambda_{D_{4}}\right)$ which we call the $D_{4}$-TORUS, whose associated Kummer surface $X_{D_{4}}$ we call the TETRAHEDRAL KUMmER SURFACE for reasons to be explained below. Its group of holomorphic symplectic automorphism, 13 is the finite group $\mathcal{T}_{192}:=\left(\mathbb{Z}_{2}\right)^{4} \rtimes A_{4}$, with $A_{4}$ the group of even permutations on 4 elements.

This subsection is devoted to the investigation of the tetrahedral Kummer surface $X_{D_{4}}$. In particular, we describe its symmetry group $\mathcal{T}_{192}$ as a subgroup of $F_{384}$, one of the 11 subgroups of $M_{24}$ which have the property that every finite group of symplectic automorphisms of a K3 surface is isomorphic to a subgroup of one of them by Mukai's theorem 3.2.5. Moreover, we construct a linear bijection $\Theta: H_{*}(X, \mathbb{Z}) \longrightarrow N(-1)$ which induces an isometry of the lattice $M_{\mathcal{T}_{192}}$ of theorem 3.3 .7 with its image which is equivariant with respect to $\mathcal{T}_{192}$.

\subsubsection{The $D_{4}$-torus and the tetrahedral Kummer surface}

As already mentioned above, we have the

\footnotetext{
${ }^{13}$ Note that the full symplectic automorphism group of the tetrahedral Kummer surface (disregarding the dual Kähler class) is infinite, see e.g. 38.
} 
Definition 4.2.1 Consider the lattice $\Lambda_{D_{4}} \subset \mathbb{C}^{2}$ which is generated by the four vectors

$$
\vec{\lambda}_{1}=(1,0), \quad \vec{\lambda}_{2}=(i, 0), \quad \vec{\lambda}_{3}=(0,1), \quad \vec{\lambda}_{4}=\frac{1}{2}(i+1, i+1) \quad \in \mathbb{C}^{2} .
$$

We call $\Lambda_{D_{4}}$ the $D_{4}$-LATtice, and $T=T\left(\Lambda_{D_{4}}\right)$ is the $D_{4}$-TORUs. Moreover, we call the Kummer surface $X_{D_{4}}$ with underlying torus $T=T\left(\Lambda_{D_{4}}\right)$, equipped with the induced dual Kähler class, which in fact yields a polarization, the TETRAHEDRAL KUMMER SURFACE.

Note that the $D_{4}$-lattice is isomorphic to the root lattice of the simple Lie algebra $\mathfrak{d}_{4}$, thus our terminology. For the tetrahedral Kummer surface $X_{D_{4}}$ our terminology is motivated by its symmetry group. Namely, according to [48, Table 9] we have

Proposition 4.2.2 The group $\mathcal{T}$ of holomorphic symplectic automorphisms of the $D_{4}$-torus has the maximal order 24 among all translation-free groups of holomorphic symplectic automorphisms of complex Kähler tori. The group $\mathcal{T}$ is the BINARY TETRAHEDRAL GROUP, and its action on the universal cover $\mathbb{C}^{2}$ of $T\left(\Lambda_{D_{4}}\right)$ with standard complex coordinates $\left(z_{1}, z_{2}\right)$ is generated by

$$
\begin{aligned}
\gamma_{1}:\left(z_{1}, z_{2}\right) & \mapsto\left(i z_{1},-i z_{2}\right), \\
\gamma_{2}:\left(z_{1}, z_{2}\right) & \mapsto\left(-z_{2}, z_{1}\right), \\
\gamma_{3}:\left(z_{1}, z_{2}\right) & \mapsto \frac{i+1}{2}\left(i\left(z_{1}-z_{2}\right),-\left(z_{1}+z_{2}\right)\right) .
\end{aligned}
$$

Note that the binary tetrahedral group $\mathcal{T}$ is a $\mathbb{Z}_{2}$-extension of the group $A_{4}$ of orientation preserving symmetries of a regular tetrahedron in $\mathbb{R}^{3}$. Indeed, the group $A_{4}$ of even permutations on 4 elements acts as a subgroup of $\mathrm{SO}(3)$, and $\mathcal{T}$ is its lift to the universal cover $\mathrm{SU}(2)$ of $\mathrm{SO}(3)$. Clearly, $\gamma_{1}$ and $\gamma_{2}$ in (4.6) have order 4 , while $\gamma_{3}$ has order 3 , and as a cross-check one confirms that the standard holomorphic $(2,0)$-form $\widehat{\Omega}=d z_{1} \wedge d z_{2}$ and the standard Kähler form $\widehat{\omega}_{T}=\frac{1}{2 i}\left(d z_{1} \wedge d \bar{z}_{1}+d z_{2} \wedge d \bar{z}_{2}\right)$ of $T\left(\Lambda_{D_{4}}\right)$ as in (3.1), (3.4) are invariant under $\mathcal{T}$. Hence indeed, $\mathcal{T}$ acts as a holomorphic symplectic automorphism group on $T\left(\Lambda_{D_{4}}\right)$. Since $\gamma_{1}^{2}=\gamma_{2}^{2}$, for example, yields the $\mathbb{Z}_{2}$-orbifold action $\left(z_{1}, z_{2}\right) \mapsto\left(-z_{1},-z_{2}\right)$ used in the Kummer construction of definition 2.2.2, the action of $\mathcal{T}$ on $T\left(\Lambda_{D_{4}}\right)$ induces a symplectic action of the tetrahedral symmetry group $A_{4}=\mathcal{T} /\{ \pm 1\}$ on the corresponding Kummer surface $X_{D_{4}}=T\left(\widetilde{\left.\Lambda_{D_{4}}\right)} / \mathbb{Z}_{2}\right.$, thus our terminology. Moreover, $\widehat{\omega}_{T}$ induces the polarization $\omega$ of this K3 surface. We have the following

Proposition 4.2.3 Consider the tetrahedral Kummer surface $X_{D_{4}}$ of definition 4.2.1 with its induced polarization, and with notations as in definition 2.2.2.

The symmetry group of $X_{D_{4}}$ is the group $\mathcal{T}_{192}:=\left(\mathbb{Z}_{2}\right)^{4} \rtimes A_{4}$, where $A_{4}$ denotes the group of even permutations on 4 elements.

By means of the Torelli Theorem 3.1.1 and with respect to our usual marking described at the end of section 2.2, the complex structure of $X_{D_{4}}$ is specified by the 2-dimensional oriented subspace $\Omega \subset H_{2}\left(X_{D_{4}}, \mathbb{R}\right)$ with orthonormal basis

$$
\begin{aligned}
& \Omega_{1}=-\pi_{*} \lambda_{12}+\pi_{*} \lambda_{13}+\pi_{*} \lambda_{23}-2 \pi_{*} \lambda_{24}, \\
& \Omega_{2}=-\pi_{*} \lambda_{12}-\pi_{*} \lambda_{13}+\pi_{*} \lambda_{23}+2 \pi_{*} \lambda_{14},
\end{aligned}
$$

where $\lambda_{i j}=\lambda_{i} \vee \lambda_{j}$ as in (2.6) with generators $\lambda_{1}, \ldots, \lambda_{4}$ of $H_{1}\left(T_{D_{4}}, \mathbb{Z}\right)$ represented by the $\vec{\lambda}_{1}, \ldots, \vec{\lambda}_{4}$ of (4.6). Furthermore, the induced polarization is

$$
\omega=\pi_{*} \lambda_{12}+\pi_{*} \lambda_{13}+\pi_{*} \lambda_{23}+2 \pi_{*} \lambda_{34} \in H_{2}\left(X_{D_{4}}, \mathbb{Z}\right) .
$$

Proof: By proposition 4.2.2, the group $G_{D_{4}}^{\prime}$ of holomorphic symplectic automorphisms of $T\left(\Lambda_{D_{4}}\right)$ that fix $0 \in \mathbb{C}^{2} / \Lambda_{D_{4}}$ is the binary tetrahedral group $G_{D_{4}}^{\prime}=\mathcal{T}$ with $G_{T}=G_{D_{4}}^{\prime} / \mathbb{Z}_{2}=$ 
$A_{4}$ by the above. Hence proposition 3.3 .4 shows that $\mathcal{T}_{192}=\left(\mathbb{Z}_{2}\right)^{4} \rtimes A_{4}$ is the holomorphic symplectic automorphism group of the tetrahedral Kummer surface. One checks that this group has order 192, thus the notation.

As explained in section 3.1, in terms of local coordinates the complex structure and polarization of $X_{D_{4}}$ are determined by $\Omega_{1}, \Omega_{2}, \omega \in H_{2}\left(X_{D_{4}}, \mathbb{R}\right)$ as given in (3.2), (3.5), where $\vec{e}_{1}, \ldots, \vec{e}_{4}$ are the standard Euclidean coordinate vectors of $\mathbb{R}^{4} \cong \mathbb{C}^{2}$. Here, indeed, we may use standard coordinates on the universal cover of $T\left(\Lambda_{D_{4}}\right)$ to induce local coordinates on $T\left(\Lambda_{D_{4}}\right) / \mathbb{Z}_{2}$ away from the singular points. The generators (4.5) of the lattice $\Lambda_{D_{4}}$ are $\vec{\lambda}_{1}=\vec{e}_{1}, \vec{\lambda}_{2}=\vec{e}_{2}, \vec{\lambda}_{3}=\vec{e}_{3}$ and $\vec{\lambda}_{4}=\frac{1}{2}\left(\vec{e}_{1}+\vec{e}_{2}+\vec{e}_{3}+\vec{e}_{4}\right)$. Inserting these expressions in (3.2), (3.5), one obtains the formulas for $\Omega_{1}, \Omega_{2}, \omega$ in terms of the images $\pi_{*} \lambda_{i j} \in H_{2}\left(X_{D_{4}}, \mathbb{Z}\right)$ of the $\lambda_{i j}$ as claimed.

By proposition 4.2.3, we in particular have $\Omega_{1}, \Omega_{2}, \omega \in H_{2}\left(X_{D_{4}}, \mathbb{Z}\right)$, such that analogously to the example of the Kummer surface $X_{0}$ with underlying torus $T_{0}=\mathbb{C}^{2} / \mathbb{Z}^{4}$ discussed in section 3.1, the results of Shioda and Inose [38] apply: The quadratic form associated to the transcendental lattice $\Omega \cap H_{2}\left(X_{D_{4}}, \mathbb{Z}\right)$ of $X_{D_{4}}$ uniquely determines the complex structure of this Kummer surface. Since generators of this lattice are given by

$$
\begin{aligned}
& I_{1}:=\frac{1}{2}\left(\Omega_{1}+\Omega_{2}\right)=-\pi_{*} \lambda_{12}+\pi_{*} \lambda_{14}+\pi_{*} \lambda_{23}-\pi_{*} \lambda_{24}, \\
& I_{2}:=\frac{1}{2}\left(\Omega_{1}-\Omega_{2}\right)=\pi_{*} \lambda_{13}-\pi_{*} \lambda_{14}-\pi_{*} \lambda_{24},
\end{aligned}
$$

the relevant quadratic form is $\left\langle I_{i}, I_{j}\right\rangle=4 \delta_{i j}$, in agreement with (3.3). In other words, $X_{D_{4}}$ and the Kummer surface $X_{0}$ constructed from the square torus $T_{0}$ share the same complex structure, and according to the final remark of [38, they agree with the elliptic modular surface of level 4 defined over $\mathbb{Q}(\sqrt{-1})$ of [39, p. 57]. However, our tetrahedral Kummer surface comes equipped with the polarization $\omega$, which is invariant under the action of the group $\mathcal{T}_{192}$ described in proposition 4.2.3. With $\Sigma:=\operatorname{span}_{\mathbb{R}}\left\{\Omega_{1}, \Omega_{2}, \omega\right\}$, the lattice $\Sigma \cap$ $H_{2}\left(X_{D_{4}}, \mathbb{Z}\right)$ has generators $I_{1}, I_{2}$ as above and

$$
I_{3}:=\frac{1}{2}\left(\Omega_{2}+\omega\right)=\pi_{*} \lambda_{14}+\pi_{*} \lambda_{23}+\pi_{*} \lambda_{34},
$$

such that the associated quadratic form is

$$
\left(\begin{array}{rrr}
4 & 0 & 2 \\
0 & 4 & -2 \\
2 & -2 & 4
\end{array}\right),
$$

in contrast to (3.6). In other words, the Kummer surfaces $X_{0}$ and $X_{D_{4}}$ carry different induced polarizations.

For later convenience we note that the following three vectors generate the lattice $\Sigma^{\perp} \cap$ $\pi_{*}\left(H_{2}\left(T\left(\Lambda_{D_{4}}\right), \mathbb{Z}\right)\right)$ of rank 3 :

$$
\begin{aligned}
I_{1}^{\perp} & :=\pi_{*} \lambda_{14}+\pi_{*} \lambda_{24}-\pi_{*} \lambda_{23}, \\
I_{2}^{\perp} & :=\pi_{*} \lambda_{13}+\pi_{*} \lambda_{24}+\pi_{*} \lambda_{34}, \\
I_{3}^{\perp} & :=-\pi_{*} \lambda_{12}+\pi_{*} \lambda_{14}+\pi_{*} \lambda_{34} .
\end{aligned}
$$

\subsubsection{The holomorphic symplectic automorphism group of the tetrahedral Kum- mer surface as a subgroup of $M_{24}$}

As was explained in section 4.1, our theorem 3.3.7 implies that the holomorphic symplectic automorphism group $\mathcal{T}_{192}$ of our tetrahedral Kummer surface $X_{D_{4}}$ also acts faithfully on the Niemeier lattice $N$ of type $A_{1}^{24}$. In the following, we calculate this action, and thereby we 
identify $\mathcal{T}_{192}$ as a subgroup of the Mathieu group $M_{24}$. Throughout, we use the same notations as in theorem 3.3.7.

Recall that the action of $\mathcal{T}_{192}$ on $N$ can be induced by a primitive embedding of the lattice $M_{\mathcal{T}_{192}}$ in $N(-1)$ such that its image $\widetilde{M}_{\mathcal{T}_{192}}$ contains one of the lattices $\widetilde{\mathcal{P}}_{n_{0}}$ of proposition 2.3.6, $n_{0} \in \mathcal{O}_{9}$. Such an embedding exists according to proposition 4.1.1, which in particular states that we may require that the embedding of the Kummer lattice $\Pi \subset M_{\mathcal{T}_{192}}$ in $N(-1)$ is induced by $E_{I(n)} \mapsto f_{n}$ for every $n \in \mathcal{I} \backslash \mathcal{O}_{9}$, by means of the map $I$ of (2.14). Moreover, according to that proposition, the involutions $\iota_{1}, \ldots, \iota_{4} \in M_{24}$ of (4.1) then yield the action which the translational automorphism group $G_{t} \cong\left(\mathbb{Z}_{2}\right)^{4}$ in $\mathcal{T}_{192}$ induces on $N$, if we impose equivariance of our embedding $i_{\mathcal{T}_{192}}: M_{\mathcal{T}_{192}} \hookrightarrow N(-1)$ with respect to $\mathcal{T}_{192}$. By proposition 4.2 .3 , the symplectic action of the group $\mathcal{T}_{192}$ on the tetrahedral Kummer surface is generated by this translational automorphism group along with a symplectic action of $A_{4}$, which is induced by the symplectic action of the binary tetrahedral group $\mathcal{T}$ on $T\left(\Lambda_{D_{4}}\right)$. With respect to standard Euclidean coordinates on $\mathbb{R}^{4} \cong \mathbb{C}^{2}$, by (4.6) the generators of the latter group are

$$
\begin{aligned}
\gamma_{1}\left(x_{1}, x_{2}, x_{3}, x_{4}\right)= & \left(-x_{2}, x_{1}, x_{4},-x_{3}\right) \\
\gamma_{2}\left(x_{1}, x_{2}, x_{3}, x_{4}\right)= & \left(-x_{3},-x_{4}, x_{1}, x_{2}\right) \\
\gamma_{3}\left(x_{1}, x_{2}, x_{3}, x_{4}\right)= & \frac{1}{2}\left(\left[-x_{1}-x_{2}+x_{3}+x_{4}\right],\left[x_{1}-x_{2}-x_{3}+x_{4}\right],\right. \\
& {\left.\left[-x_{1}+x_{2}-x_{3}+x_{4}\right],\left[-x_{1}-x_{2}-x_{3}-x_{4}\right]\right) . }
\end{aligned}
$$

On the generators $\vec{\lambda}_{1}, \ldots, \vec{\lambda}_{4}$ of $\Lambda_{D_{4}}$ given in (4.5) we thus have

$$
\begin{array}{rll}
\gamma_{1}: & \vec{\lambda}_{1} \mapsto \vec{\lambda}_{2}, & \vec{\lambda}_{2} \mapsto-\vec{\lambda}_{1}, \quad \vec{\lambda}_{3} \mapsto-2 \vec{\lambda}_{4}+\vec{\lambda}_{1}+\vec{\lambda}_{2}+\vec{\lambda}_{3}, \\
& & \vec{\lambda}_{4} \mapsto-\vec{\lambda}_{4}+\vec{\lambda}_{2}+\vec{\lambda}_{3}, \\
\gamma_{2}: & \vec{\lambda}_{1} \mapsto \vec{\lambda}_{3}, & \vec{\lambda}_{2} \mapsto 2 \vec{\lambda}_{4}-\vec{\lambda}_{1}-\vec{\lambda}_{2}-\vec{\lambda}_{3}, \\
& \vec{\lambda}_{3} \mapsto-\vec{\lambda}_{1}, & \vec{\lambda}_{4} \mapsto \vec{\lambda}_{4}-\vec{\lambda}_{1}-\vec{\lambda}_{2}, \\
\gamma_{3}: & \vec{\lambda}_{1} \mapsto \vec{\lambda}_{2}-\vec{\lambda}_{4}, & \vec{\lambda}_{2} \mapsto \vec{\lambda}_{3}-\vec{\lambda}_{4}, \\
& \vec{\lambda}_{3} \mapsto \vec{\lambda}_{1}-\vec{\lambda}_{4}, & \vec{\lambda}_{4} \mapsto-2 \vec{\lambda}_{4}+\vec{\lambda}_{1}+\vec{\lambda}_{2}+\vec{\lambda}_{3} .
\end{array}
$$

These transformations induce permutations of the singular points in $T\left(\Lambda_{D_{4}}\right) / \mathbb{Z}_{2}$ and thus affine linear maps $\left[\gamma_{1}\right],\left[\gamma_{2}\right],\left[\gamma_{3}\right]$ on the hypercube $\mathbb{F}_{2}^{4}$ which labels the $E_{\vec{a}} \in \Pi$ of proposition 2.2.3. For the induced permutations $\widehat{\gamma}_{k}$ on $\mathcal{I} \backslash \mathcal{O}_{9}$, imposing equivariance of our embedding $i_{\mathcal{T}_{192}}$ then amounts to the condition $\widehat{\gamma}_{k}(n)=\left[\gamma_{k}\right](I(n))$ for all $n \in \mathcal{I} \backslash \mathcal{O}_{9}$. We obtain

$$
\begin{aligned}
& \widehat{\gamma}_{1}=(2,8)(7,18)(10,22)(11,13)(12,17)(14,20) \\
& \widehat{\gamma}_{2}=(2,18)(7,8)(10,17)(11,14)(12,22)(13,20), \\
& \widehat{\gamma}_{3}=(2,12,13)(4,16,21)(7,17,20)(8,22,14)(10,11,18) .
\end{aligned}
$$

These permutations must be accompanied by appropriate permutations $\sigma_{k}, k \in\{1,2,3\}$, of the labels in $\mathcal{O}_{9}$ in order to yield automorphisms of the Niemeier lattice $N$ or equivalently of the Golay code $\mathcal{G}_{24}$. However, one checks that for each $\widehat{\gamma}_{k}$, there exists a unique permutation $\sigma_{k}$ of $\mathcal{O}_{9}$ yielding $\gamma_{k}:=\sigma_{k} \circ \widehat{\gamma}_{k} \in M_{24}$. Altogether we obtain

Proposition 4.2.4 Consider the tetrahedral Kummer surface $X_{D_{4}}$ of definition 4.2 .1 with its induced polarization. Let $i_{\mathcal{T}_{192}}: M_{\mathcal{T}_{192}} \hookrightarrow N(-1)$ denote a primitive embedding of the type constructed in proposition 4.1.1. This embedding is equivariant with respect to the holomorphic 
symplectic automorphism group $\mathcal{T}_{192}$, where the action of this group on $N$ is generated by

$$
\begin{aligned}
\iota_{1} & =(1,11)(2,22)(4,20)(7,12)(8,17)(10,18)(13,21)(14,16), \\
\iota_{2} & =(1,13)(2,12)(4,14)(7,22)(8,10)(11,21)(16,20)(17,18), \\
\iota_{3} & =(1,14)(2,17)(4,13)(7,10)(8,22)(11,16)(12,18)(20,21), \\
\iota_{4} & =(1,17)(2,14)(4,12)(7,20)(8,11)(10,21)(13,18)(16,22), \\
\gamma_{1} & =(2,8)(7,18)(9,24)(10,22)(11,13)(12,17)(14,20)(15,19), \\
\gamma_{2} & =(2,18)(7,8)(9,19)(10,17)(11,14)(12,22)(13,20)(15,24), \\
\gamma_{3} & =(2,12,13)(4,16,21)(7,17,20)(8,22,14)(9,19,24)(10,11,18) .
\end{aligned}
$$

In particular, since $f_{n_{0}}$ is invariant under the action of $\mathcal{T}_{192}$, we find $n_{0} \in\{3,5,6,23\}$.

The above proposition identifies $\mathcal{T}_{192}$ as a subgroup of $M_{24}$ which leaves the octad $\mathcal{O}_{9}=$ $\{9,5,24,19,23,3,6,15\}$ invariant. This group may be constructed as a succession of stabilizers of the Mathieu group $M_{24}$, starting with the stabilizer in $M_{24}$ of the element $5 \in \mathcal{I}$, which is $M_{23}$, followed by the stabilizer in $M_{23}$ of the element $3 \in \mathcal{I}$, which is $M_{22}$. The next step is to construct the stabilizer in $M_{22}$ of the element $6 \in \mathcal{I}$, which is $\operatorname{PSL}(3,4)$, and the stabilizer in $\operatorname{PSL}(3,4)$ of the element $23 \in \mathcal{I}$, whose structure is $\left(\mathbb{Z}_{2}\right)^{4} \rtimes A_{5}$, and finally to obtain the stabilizer in that group of the set $\{9,15,19,24\}$. This last stabilizer group has order 192 and coincides with the copy of $\mathcal{T}_{192}$ generated above. Equivalently, one obtains $\mathcal{T}_{192}$ from $M_{23}$, the stabilizer of $5 \in \mathcal{I}$, by first stabilizing the set $\mathcal{O}_{9}$. This yields the maximal subgroup $\left(\mathbb{Z}_{2}\right)^{4} \rtimes A_{7}$ of $M_{23}$ which we will recover in section 4.4. Then successively stabilizing 3,6, $23 \in \mathcal{I}$ in that group one obtains the (maximal) subgroups $\left(\mathbb{Z}_{2}\right)^{4} \rtimes A_{6},\left(\mathbb{Z}_{2}\right)^{4} \rtimes A_{5},\left(\mathbb{Z}_{2}\right)^{4} \rtimes A_{4}=\mathcal{T}_{192}$ of $M_{22}, \operatorname{PSL}(3,4),\left(\mathbb{Z}_{2}\right)^{4} \rtimes A_{5}$, respectively.

We remark that the Mathieu group $M_{24}$ may be generated from the set of 7 permutations given in (4.13), augmented by one extra involution, for instance

$$
\iota_{5}=(1,9)(2,5)(3,19)(4,15)(6,22)(7,18)(8,20)(10,17)(11,12)(13,16)(14,24)(21,23) .
$$

This involution is one of the 7 involutions that are seen on the Klein map, and that generate $M_{24}$ according to [49].

Now recall our discussion in section 4.1 leading to (4.2)-(4.4), the consistency conditions for an extension of the $G_{t}$-equivariant embedding $i_{\mathcal{T}_{192}}$ of $M_{\mathcal{T}_{192}}$ in $N(-1)$ to a linear bijection $\Theta: H_{*}(X, \mathbb{Z}) \longrightarrow N(-1)$. By proposition 4.2.4 the embedding $i_{\mathcal{T}_{192}}$ is actually equivariant with respect to the larger group $\mathcal{T}_{192}$ and imposes an isometry of $M_{\mathcal{T}_{192}} \cap \mathcal{K}$ with its image. This amounts to the condition that the map (4.2) is equivariant with respect to the action of $A_{4}$ induced by (4.6) on the $\pi_{*} \lambda_{i j} \in H_{2}\left(X_{D_{4}}, \mathbb{Z}\right)$ and by the $\gamma_{k}$ of (4.13) on our choices of $Q_{i j}$ from (4.3). Thus, next we need to determine the action of each $\gamma_{k}$ induced from (4.11) on the $\lambda_{i j}$ :

$$
\begin{aligned}
& \left(\begin{array}{l}
\gamma_{1}\left(\lambda_{12}\right) \\
\gamma_{1}\left(\lambda_{13}\right) \\
\gamma_{1}\left(\lambda_{14}\right) \\
\gamma_{1}\left(\lambda_{23}\right) \\
\gamma_{1}\left(\lambda_{24}\right) \\
\gamma_{1}\left(\lambda_{34}\right)
\end{array}\right)=\left(\begin{array}{cccccc}
1 & 0 & 0 & 0 & 0 & 0 \\
-1 & 0 & 0 & 1 & -2 & 0 \\
0 & 0 & 0 & 1 & -1 & 0 \\
-1 & -1 & 2 & 0 & 0 & 0 \\
-1 & -1 & 1 & 0 & 0 & 0 \\
1 & 1 & -1 & 0 & 1 & 1
\end{array}\right)\left(\begin{array}{c}
\lambda_{12} \\
\lambda_{13} \\
\lambda_{14} \\
\lambda_{23} \\
\lambda_{24} \\
\lambda_{34}
\end{array}\right), \\
& \left(\begin{array}{l}
\gamma_{2}\left(\lambda_{12}\right) \\
\gamma_{2}\left(\lambda_{13}\right) \\
\gamma_{2}\left(\lambda_{14}\right) \\
\gamma_{2}\left(\lambda_{23}\right) \\
\gamma_{2}\left(\lambda_{24}\right) \\
\gamma_{2}\left(\lambda_{34}\right)
\end{array}\right)=\left(\begin{array}{cccccc}
0 & 1 & 0 & 1 & 0 & 2 \\
0 & 1 & 0 & 0 & 0 & 0 \\
0 & 1 & 0 & 1 & 0 & 1 \\
-1 & -1 & 2 & 0 & 0 & 0 \\
0 & -1 & 1 & -1 & 1 & -1 \\
1 & 0 & -1 & 0 & 0 & 0
\end{array}\right)\left(\begin{array}{c}
\lambda_{12} \\
\lambda_{13} \\
\lambda_{14} \\
\lambda_{23} \\
\lambda_{24} \\
\lambda_{34}
\end{array}\right),
\end{aligned}
$$




$$
\left(\begin{array}{c}
\gamma_{3}\left(\lambda_{12}\right) \\
\gamma_{3}\left(\lambda_{13}\right) \\
\gamma_{3}\left(\lambda_{14}\right) \\
\gamma_{3}\left(\lambda_{23}\right) \\
\gamma_{3}\left(\lambda_{24}\right) \\
\gamma_{3}\left(\lambda_{34}\right)
\end{array}\right)=\left(\begin{array}{cccccc}
0 & 0 & 0 & 1 & -1 & 1 \\
-1 & 0 & 1 & 0 & -1 & 0 \\
-1 & 0 & 1 & 1 & -1 & 1 \\
0 & -1 & 1 & 0 & 0 & -1 \\
0 & -1 & 1 & -1 & 1 & -1 \\
1 & 1 & -1 & 0 & 1 & 1
\end{array}\right)\left(\begin{array}{c}
\lambda_{12} \\
\lambda_{13} \\
\lambda_{14} \\
\lambda_{23} \\
\lambda_{24} \\
\lambda_{34}
\end{array}\right) .
$$

The task now is to make a choice for each $Q_{i j}$ in (4.3) such that in the map (4.2) there exists a choice of signs making it equivariant under $\gamma_{1}, \gamma_{2}, \gamma_{3}$. A first step towards a solution to this task, though not uniquely determined at this stage, is

Proposition 4.2.5 The following choices in (4.3) yield a map $\bar{I}\left(\pi_{*} \lambda_{i j}\right):=Q_{i j}$ with $\bar{I}(\lambda+$ $\left.\lambda^{\prime}\right):=\bar{I}(\lambda)+\bar{I}\left(\lambda^{\prime}\right)$ by means of symmetric differences of sets which for each $k=1,2,3$ obeys $\bar{I}\left(\pi_{*} \gamma_{k}\left(\lambda_{i j}\right)\right)=\gamma_{k}\left(Q_{i j}\right)$ for all labels $i j$ :

$$
\begin{array}{ll}
Q_{12}=\{3,6,15,19\}, & Q_{34}=\{6,9,15,19\}, \\
Q_{13}=\{6,15,23,24\}, & Q_{24}=\{15,19,23,24\}, \\
Q_{14}=\{3,9,15,24\}, & Q_{23}=\{3,9,15,23\} .
\end{array}
$$

All these $Q_{i j}$ avoid the label $n_{0}:=5 \in \mathcal{O}_{9}$.

In section 4.4 we shall show that there exists a linear bijection $\Theta: H_{*}(X, \mathbb{Z}) \longrightarrow N(-1)$ which is compatible with the overarching finite symmetry group $\left(\mathbb{Z}_{2}\right)^{4} \rtimes A_{7}$ of definition 3.3.5 in the following sense: On two distinct sublattices $M_{G}$ of rank 20 of $H_{*}(X, \mathbb{Z})$ the map $\Theta$ restricts to isometric embeddings $M_{G} \hookrightarrow N(-1)$ that are equivariant with respect to appropriate holomorphic symplectic automorphism groups $G$ that generate $\left(\mathbb{Z}_{2}\right)^{4} \rtimes A_{7}$. Under both groups $G$, the vector $v_{0}-v \in M_{G}$ is invariant. It follows that in order for such a map $\Theta$ to exist, there must be a label $n_{0} \in \mathcal{O}_{9}$ which occurs in none of the $Q_{i j}$. Indeed, $f_{n_{0}}$ is the image of $v_{0}-v$ under the embedding $i_{\mathcal{T}_{192}}$. Since the symmetry group of our Kummer surface stabilizes the generators $v_{0}$ and $v$ of $H_{0}(X, \mathbb{Z})$ and $H_{4}(X, \mathbb{Z})$, and since $i_{\mathcal{T}_{192}}$ is equivariant with respect to $\mathcal{T}_{192}$, the root $f_{n_{0}}$ must be stabilized by $\mathcal{T}_{192}$. If $f_{n_{0}}$ is stabilized by the induced action of the entire overarching finite symmetry group, then $f_{n_{0}}$ cannot occur in any of the $q_{i j}$. In other words, the label $n_{0} \in \mathcal{O}_{9}$ cannot occur in any of the $Q_{i j}$.

\subsubsection{A linear bijection between even unimodular lattices}

For the tetrahedral Kummer surface $X_{D_{4}}$ of definition 4.2 .1 with holomorphic symplectic automorphism group $\mathcal{T}_{192}$, in the following we shall show that the primitive embedding $i_{\mathcal{T}_{192}}: M_{\mathcal{T}_{192}} \hookrightarrow N(-1)$ of proposition 4.1.1, which yields $E_{\vec{a}} \mapsto f_{I^{-1}(\vec{a})}$ for each $\vec{a} \in \mathbb{F}_{2}^{4}$ and $v_{0}-v \mapsto f_{n_{0}}$, can be extended to a linear bijection $\Theta: H_{*}(X, \mathbb{Z}) \longrightarrow N(-1)$ which obeys (4.2) with the choices of proposition 4.2.5 and with $n_{0}=5$.

First, by proposition 4.2.5 the map $\bar{I}$ yields

$$
\begin{aligned}
& \bar{I}\left(\pi_{*} \lambda_{12}+\pi_{*} \lambda_{34}+\pi_{*} \lambda_{14}\right)=\{15,24\}, \\
& \bar{I}\left(\pi_{*} \lambda_{12}+\pi_{*} \lambda_{34}+\pi_{*} \lambda_{23}\right)=\{15,23\}, \\
& \bar{I}\left(\pi_{*} \lambda_{13}+\pi_{*} \lambda_{24}+\pi_{*} \lambda_{12}\right)=\{3,15\}, \\
& \bar{I}\left(\pi_{*} \lambda_{13}+\pi_{*} \lambda_{24}+\pi_{*} \lambda_{34}\right)=\{9,15\}, \\
& \bar{I}\left(\pi_{*} \lambda_{14}+\pi_{*} \lambda_{23}+\pi_{*} \lambda_{13}\right)=\{6,15\}, \\
& \bar{I}\left(\pi_{*} \lambda_{14}+\pi_{*} \lambda_{23}+\pi_{*} \lambda_{24}\right)=\{15,19\} .
\end{aligned}
$$

Therefore, if a map $\Theta$ exists as claimed, then it obeys $\Theta: \pi_{*} \lambda_{12} \pm \pi_{*} \lambda_{34} \pm \pi_{*} \lambda_{14} \mapsto \pm f_{15} \pm f_{24}$, etc., with four signs to be chosen for each such identification. If we need to relax the constraint 
posed in (4.2), namely that every $\frac{1}{2} \pi_{*} \lambda_{i j}$ is mapped to a representative of an element in $\widetilde{\mathcal{K}}_{n_{0}}^{*} / \widetilde{\mathcal{K}}_{n_{0}}$ of minimal length, then additional summands of the form $2 \Delta$ with $\Delta \in \widetilde{K}$ may occur. Our choices of signs are severely restricted by imposing (4.2), and one checks that the following yields a lift of (4.4) which is consistent with all gluing prescriptions, with (4.2) as well as the above (4.15)

$$
\begin{aligned}
& I_{3}^{\perp}=-\pi_{*} \lambda_{12}+\pi_{*} \lambda_{34}+\pi_{*} \lambda_{14} \longmapsto f_{24}-f_{15}, \\
& J_{1}^{\perp}-I_{3}^{\perp}=\pi_{*} \lambda_{12}-\pi_{*} \lambda_{34}-\pi_{*} \lambda_{23} \longmapsto f_{15}-f_{23}, \\
& -J_{3}^{\perp}+I_{2}^{\perp}=\pi_{*} \lambda_{13}+\pi_{*} \lambda_{24}+\pi_{*} \lambda_{12} \longmapsto f_{3}-f_{15}, \\
& I_{2}^{\perp}=\pi_{*} \lambda_{13}+\pi_{*} \lambda_{24}+\pi_{*} \lambda_{34} \longmapsto f_{9}-f_{15}, \\
& J_{2}^{\perp}-I_{1}^{\perp}=-\pi_{*} \lambda_{14}+\pi_{*} \lambda_{23}+\pi_{*} \lambda_{13} \longmapsto f_{15}-f_{6}, \\
& I_{1}^{\perp}=\pi_{*} \lambda_{14}-\pi_{*} \lambda_{23}+\pi_{*} \lambda_{24} \longmapsto f_{19}-f_{15},
\end{aligned}
$$

(see (4.9) and (4.21) for the definitions of the $I_{k}^{\perp}$ and $J_{k}^{\perp}$ ), or equivalently

$$
\begin{array}{rlrr}
\Theta: \pi_{*} \lambda_{12} \longmapsto 2 q_{12}= & f_{3}+f_{6}-f_{15}-f_{19}, \\
\Theta: \pi_{*} \lambda_{34} \longmapsto 2 q_{34}= & f_{6}+f_{9}-f_{15}-f_{19}, \\
\Theta: \pi_{*} \lambda_{13} \longmapsto 2 q_{13}= & -f_{6}+f_{15}-f_{23}+f_{24}, \\
\Theta: \pi_{*} \lambda_{24} \longmapsto 2 q_{24}= & -f_{15}+f_{19}+f_{23}-f_{24}, \\
\Theta: \pi_{*} \lambda_{14} \longmapsto 2 q_{14}= & f_{3}-f_{9}-f_{15}+f_{24}, \\
\Theta: \pi_{*} \lambda_{23} \longmapsto 2 q_{23}= & f_{3}-f_{9}-f_{15}+f_{23},
\end{array}
$$

where $2 q_{i j}=\sum_{n \in Q_{i j}}( \pm) f_{n}$ in accord with (4.2) and (4.14). In (4.20) we will see that this choice of signs allows us to isometrically identify the lattice generated by $I_{1}^{\perp}, I_{2}^{\perp}, I_{3}^{\perp} \in M_{\mathcal{T}_{192}}$ with its image in $N(-1)$. In particular, the choices of signs on the left hand side of (4.16) are already fixed by enforcing this property together with (4.2). Similarly, in (4.27) we will see that our choice of signs also allows us to isometrically identify the lattice generated by $J_{1}^{\perp}, J_{2}^{\perp}, J_{3}^{\perp} \in M_{\mathcal{T}_{64}}$ with its image in $N(-1)$. Our choice of signs is unique with these properties, up to the freedom for each $n \in \mathcal{I}$ to replace $f_{n}$ by $-f_{n}$ everywhere, which induces an isometry of $N$ according to proposition 2.3.2. This freedom of choice is in accord with the fact that we are actually interested in $M_{24}$, where by proposition 2.3 .2 we have $M_{24}=$ $\operatorname{Aut}(N) /\left(\mathbb{Z}_{2}\right)^{24}$, with $\left(\mathbb{Z}_{2}\right)^{24}$ implementing precisely the freedom of choice of replacing any $f_{n}$ by $-f_{n}$ everywhere.

Finally, with our choice $n_{0}=5$ from proposition 4.2.5, $\Theta\left(v_{0}-v\right)=f_{5}$, and (4.2) lifts to

$$
\Theta: v_{0}+v \longmapsto f_{3}+f_{6}+f_{9}-f_{15}-f_{19}-f_{23}-f_{24} .
$$

The signs on the right hand side are arbitrary, up to the fact that the right hand side together with the $q_{i j}$ in (4.17) must generate the lattice $\widetilde{\mathcal{K}}_{n_{0}}$ of proposition 2.3.6 with $n_{0}=5, \widetilde{\mathcal{K}}_{5}=$ $\operatorname{span}_{\mathbb{Z}}\left\{f_{3}, f_{6}, f_{9}, f_{15}, f_{19}, f_{23}, f_{24}\right\}$ (see the proof of proposition 2.3.6). We thus have

$$
\begin{aligned}
\Theta: v_{0} & \longmapsto \frac{1}{2}\left(f_{3}+f_{5}+f_{6}+f_{9}-f_{15}-f_{19}-f_{23}-f_{24}\right), \\
\Theta: v & \longmapsto \frac{1}{2}\left(f_{3}-f_{5}+f_{6}+f_{9}-f_{15}-f_{19}-f_{23}-f_{24}\right) .
\end{aligned}
$$

Collecting the various ingredients, we claim

Theorem 4.2.6 Consider the tetrahedral Kummer surface $X_{D_{4}}$ of definition 4.2 .1 with its induced polarization and the symmetry group $\mathcal{T}_{192}$. 
There exists a linear bijection $\Theta: H_{*}(X, \mathbb{Z}) \longrightarrow N(-1)$ which is induced by (4.17) and (4.18) along with the map I of (2.14) which induces

$$
\Theta: E_{\vec{a}} \longmapsto f_{I^{-1}(\vec{a})} \quad \text { for every } \vec{a} \in \mathbb{F}_{2}^{4} .
$$

The map $\Theta$ isometrically embeds the lattice $M_{\mathcal{T}_{192}}$ of theorem 3.3 .7 into $N(-1)$, such that the restriction $i_{\mathcal{T}_{192}}$ of $\Theta$ to $M_{\mathcal{T}_{192}}$ yields an embedding $i_{\mathcal{T}_{192}}: M_{\mathcal{T}_{192}} \hookrightarrow N(-1)$ of the type constructed in proposition 4.1.1. Moreover, $i_{\mathcal{T}_{192}}$ is equivariant with respect to the group $\mathcal{T}_{192}$, where on $H_{*}(X, \mathbb{Z})$ the action of $\mathcal{T}_{192}$ is induced by its action on $X_{D_{4}}$ as holomorphic symplectic automorphism group, and on the Niemeier lattice $N$ of type $A_{1}^{24}$ its action is generated by $\iota_{1}, \ldots, \iota_{4}, \gamma_{1}, \gamma_{2}, \gamma_{3}$ as in (4.13). The lattice $M_{\mathcal{T}_{192}}$ has rank 20.

Proof: We first need to show that (4.17), (4.18), (4.19) can be extended to a linear bijection $\Theta: H_{*}(X, \mathbb{Z}) \longrightarrow N(-1)$. With notations as in proposition 2.3.6 and using (4.16) along with (4.18) we see that $\Theta(\mathcal{K})=\widetilde{\mathcal{K}}_{5}$. Similarly, (4.19) implies $\Theta(\Pi)=\widetilde{\Pi}$ according to the proof of proposition 2.3.4, and since $\Theta\left(v_{0}-v\right)=f_{5}$ by (4.18) we find $\Theta(\mathcal{P})=\widetilde{\mathcal{P}}_{5}$. By proposition 2.2.4 we can now glue $H_{*}(X, \mathbb{Z})$ from the sublattices $\mathcal{K}$ and $\mathcal{P}$, while by proposition 2.3.6 we can glue $N$ from the sublattices $\widetilde{\mathcal{K}}_{5}$ and $\widetilde{\mathcal{P}}_{5}$. By the discussion following proposition 4.1.1, the linear bijection $\Theta: \mathcal{K} \oplus \mathcal{P} \longrightarrow \widetilde{\mathcal{K}}_{5} \oplus \widetilde{\mathcal{P}}_{5}(-1)$ is compatible with this gluing if and only if it respects (4.2). We have ensured that this is the case by construction, namely by (4.17) and (4.18). Hence under gluing, $\Theta$ extends to a linear bijection $\Theta: H_{*}(X, \mathbb{Z}) \longrightarrow N(-1)$ as claimed.

Next we claim that $\Theta$ induces a primitive embedding $i_{\mathcal{T}_{192}}: M_{\mathcal{T}_{192}} \hookrightarrow N(-1)$ of the type constructed in proposition 4.1.1. By proposition [3.3.6, the lattice $M_{\mathcal{T}_{192}}$ is the orthogonal complement of the lattice $\pi_{*}\left(H_{2}\left(T\left(\Lambda_{D_{4}}\right), \mathbb{Z}\right)^{\mathcal{T}}\right) \oplus \operatorname{span}_{\mathbb{Z}}\left\{v_{0}+v\right\}$, where $\mathcal{T}$ is the binary tetrahedral group acting as non-translational holomorphic symplectic automorphism group of the $D_{4}$-torus. By proposition 4.2 .3 together with (4.7), (4.8), the lattice $\pi_{*}\left(H_{2}\left(T\left(\Lambda_{D_{4}}\right), \mathbb{Z}\right)^{\mathcal{T}}\right)$ is generated by $I_{1}, I_{2}, I_{3}$ as defined there. Hence $M_{\mathcal{T}_{192}}$ consists of

$$
\operatorname{span}_{\mathbb{Z}}\left\{I_{1}^{\perp}, I_{2}^{\perp}, I_{3}^{\perp}\right\} \oplus \mathcal{P}
$$

with $I_{k}^{\perp}, k=1,2,3$ as in (4.9), along with the appropriate rational combinations of contributions from $K$ and $\Pi$ obtained by our gluing for generic Kummer surfaces. In particular, $M_{\mathcal{T}_{192}}$ has rank 20. We need to show that $\Theta$ maps this lattice isometrically to a primitive sublattice of $N(-1)$. We already know that $\Theta(\mathcal{P})=\widetilde{\mathcal{P}}_{5}(-1)$ isometrically, due to the above and the proof of proposition 2.3.4. Since we also know that $\Theta$ is compatible with the gluing of $H_{*}(X, \mathbb{Z})$ and $N$ from $\mathcal{P}$ and $\widetilde{\mathcal{P}}_{5}$ and their orthogonal complements, it suffices to show that the lattice generated by the $I_{k}^{\perp}, k \in\{1,2,3\}$, is isometric to the lattice generated by the three vectors $\Theta\left(I_{k}^{\perp}\right), k \in\{1,2,3\}$, up to an inversion of signature. In fact, note that by (4.16) we have

$$
I_{1}^{\perp} \longmapsto f_{19}-f_{15}, \quad I_{2}^{\perp} \longmapsto f_{9}-f_{15}, \quad I_{3}^{\perp} \longmapsto f_{24}-f_{15},
$$

yielding the quadratic form of the corresponding lattices with respect to these generators as

$$
\left(\begin{array}{lll}
-4 & -2 & -2 \\
-2 & -4 & -2 \\
-2 & -2 & -4
\end{array}\right) \longmapsto\left(\begin{array}{lll}
4 & 2 & 2 \\
2 & 4 & 2 \\
2 & 2 & 4
\end{array}\right)
$$

on both sides, as required.

Finally, equivariance with respect to $\mathcal{T}_{192}$ follows by construction for the isometric embedding $i_{\mathcal{T}_{192}}: M_{\mathcal{T}_{192}} \hookrightarrow N(-1)$ obtained by restricting $\Theta$ to $M_{\mathcal{T}_{192}}$, see proposition 4.2.4. 
Note that the comparison of the quadratic forms in (4.20) uniquely determines the relative signs in front of $f_{15}$ in the formulas for the $I_{k}^{\perp}, k \in\{1,2,3\}$, in (4.16). Note furthermore that the remaining choices of signs in (4.16) amount to choosing one sign for each $f_{n}$ separately in the formulas for the $I_{k}^{\perp}$ as well as in the identifications

$$
\begin{aligned}
& J_{1}^{\perp}:=\pi_{*} \lambda_{14}-\pi_{*} \lambda_{23} \longmapsto f_{24}-f_{23}, \\
& J_{2}^{\perp}:=\pi_{*} \lambda_{13}+\pi_{*} \lambda_{24} \longmapsto f_{19}-f_{6}, \\
& J_{3}^{\perp}:=\pi_{*} \lambda_{34}-\pi_{*} \lambda_{12} \longmapsto f_{9}-f_{3} .
\end{aligned}
$$

We wish to emphasize that our gluing strategy leading to theorem 4.2.6 differs from that used by Kondo in [10], which was explained in section 3.2. Indeed, due to our theorem 3.3.7, instead of basing our construction on the lattices $L_{G}$ and $N_{G}$ which Kondo uses, we can work with the lattice $M_{G}$ which contains $L_{G}$ and exceeds it by rank 2 . In particular, $M_{G}$ contains the Kummer lattice $\Pi$, which simplifies our analysis greatly. Since the vector $e=\frac{1}{2} \sum_{\vec{a} \in \mathbb{F}_{2}^{4}} E_{\vec{a}} \in \Pi$ is invariant under every holomorphic symplectic automorphism of our Kummer surface (with its induced dual Kähler class), the Kummer lattice can never be contained in Kondo's lattice $L_{G}$. In this sense, our techniques improve Kondo's construction for all Kummer surfaces with induced dual Kähler class.

\subsection{The square Kummer surface}

Let us now carry out a similar analysis as for the tetrahedral Kummer surface $X_{D_{4}}$ for another example of an algebraic Kummer surface:

Definition 4.3.1 We call the standard torus $T_{0}=T\left(\Lambda_{0}\right)=\mathbb{C}^{2} / \Lambda_{0}$ the SQUARE TORUS, where the generators of the lattice $\Lambda_{0}$ are given by

$$
\vec{\lambda}_{1}=(1,0), \quad \vec{\lambda}_{2}=(i, 0), \quad \vec{\lambda}_{3}=(0,1), \quad \vec{\lambda}_{4}=(0, i) .
$$

The Kummer surface with underlying torus $T_{0}$ is denoted by $X_{0}:=\widetilde{T_{0} / \mathbb{Z}_{2}}$, and we call it the SQUARE KUMMER SURFACE.

In the example discussed around (3.3) and (3.6) we already found

Proposition 4.3.2 Consider the square Kummer surface $X_{0}$ of definition 4.3.1, where we are working with our usual marking described at the end of section 2.2 .

The real homology classes $\Omega_{1}, \Omega_{2}, \omega$ which determine the complex structure of $X_{0}$, by means of the Torelli Theorem 3.1.1, and the induced dual Kähler class, which in fact yields a polarization, are given by

$$
\Omega_{1}=\pi_{*} \lambda_{13}-\pi_{*} \lambda_{24}, \quad \Omega_{2}=\pi_{*} \lambda_{14}+\pi_{*} \lambda_{23}, \quad \omega=\pi_{*} \lambda_{12}+\pi_{*} \lambda_{34} \in H\left(X_{0}, \mathbb{Z}\right) .
$$

Furthermore, the 3-dimensional subspace $\Sigma$ of $H_{2}\left(X_{0}, \mathbb{R}\right)$ containing these three 2-cycles yields a lattice $\Sigma \cap H_{2}\left(X_{0}, \mathbb{Z}\right)$ of (the maximal possible) rank 3 , with quadratic form

$$
\left(\begin{array}{lll}
4 & 0 & 0 \\
0 & 4 & 0 \\
0 & 0 & 4
\end{array}\right) .
$$

One checks that the group of non-translational holomorphic symplectic automorphisms of the standard torus $T_{0}$ is the binary dihedral group $\mathcal{O}$ of order 8 mentioned in the proof of proposition 3.3.4, which is generated by the symmetries

$$
\begin{aligned}
& \alpha_{1}:\left(z_{1}, z_{2}\right) \mapsto\left(i z_{1},-i z_{2}\right), \\
& \alpha_{2}:\left(z_{1}, z_{2}\right) \mapsto\left(-z_{2}, z_{1}\right)
\end{aligned}
$$


on the universal cover $\mathbb{C}^{2}$ of $T_{0}$, and which induces an action of $\left(\mathbb{Z}_{2}\right)^{2}=\mathcal{O} / \mathbb{Z}_{2}$ on the Kummer surface $X_{0}$ obtained from $T_{0}$ (see [48, Table 9] or e.g. [50, Thm.7.3.12]). By proposition 3.3.4 it then follows that the holomorphic symplectic automorphism group of $X_{0}$ is the group $G_{t} \rtimes\left(\mathbb{Z}_{2}\right)^{2}$ of order 64 , where $G_{t} \cong\left(\mathbb{Z}_{2}\right)^{4}$ is the translational automorphism group of definition 3.3 .2 .

Note that in terms of standard Euclidean coordinates of $\mathbb{C}^{2}$, the symmetries $\alpha_{1}$ and $\alpha_{2}$ agree with $\gamma_{1}$, respectively $\gamma_{2}$ in (4.6). However, the induced actions on the lattice $H_{2}\left(T_{0}, \mathbb{Z}\right)$ and thereby on $H_{*}\left(X_{0}, \mathbb{Z}\right)$ are different, since the defining lattices of the underlying tori are different. Indeed, one immediately checks that the induced actions of $\alpha_{1}$ and $\alpha_{2}$ on the generators $\vec{\lambda}_{1}, \ldots, \vec{\lambda}_{4}$ of $\Lambda_{0}$ as in (4.22) are the following:

$$
\begin{aligned}
& \alpha_{1}: \quad \vec{\lambda}_{1} \mapsto \vec{\lambda}_{2}, \quad \vec{\lambda}_{2} \mapsto-\vec{\lambda}_{1}, \quad \vec{\lambda}_{3} \mapsto-\vec{\lambda}_{4}, \quad \vec{\lambda}_{4} \mapsto \vec{\lambda}_{3}, \\
& \alpha_{2}: \quad \vec{\lambda}_{1} \mapsto \vec{\lambda}_{3}, \quad \vec{\lambda}_{2} \mapsto \vec{\lambda}_{4}, \quad \vec{\lambda}_{3} \mapsto-\vec{\lambda}_{1}, \quad \vec{\lambda}_{4} \mapsto-\vec{\lambda}_{2} .
\end{aligned}
$$

We therefore obtain

Proposition 4.3.3 Consider the square Kummer surface $X_{0}$ of definition 4.3.1, equipped with the induced polarization.

The symmetry group of $X_{0}$ is the group $\mathcal{T}_{64}:=G_{t} \rtimes\left(\mathbb{Z}_{2}\right)^{2}$ of order 64 , where $G_{t} \cong\left(\mathbb{Z}_{2}\right)^{4}$ is the translational automorphism group of definition 3.3.2. The induced action of the nontranslational quotient group $\left(\mathbb{Z}_{2}\right)^{2}$ on $H_{2}\left(T_{0}, \mathbb{Z}\right)$ with respect to the basis $\lambda_{i j}$ of (4.17) is generated by

$$
\begin{gathered}
\left(\begin{array}{l}
\alpha_{1}\left(\lambda_{12}\right) \\
\alpha_{1}\left(\lambda_{13}\right) \\
\alpha_{1}\left(\lambda_{14}\right) \\
\alpha_{1}\left(\lambda_{23}\right) \\
\alpha_{1}\left(\lambda_{24}\right) \\
\alpha_{1}\left(\lambda_{34}\right)
\end{array}\right)=\left(\begin{array}{cccccc}
1 & 0 & 0 & 0 & 0 & 0 \\
0 & 0 & 0 & 0 & -1 & 0 \\
0 & 0 & 0 & 1 & 0 & 0 \\
0 & 0 & 1 & 0 & 0 & 0 \\
0 & -1 & 0 & 0 & 0 & 0 \\
0 & 0 & 0 & 0 & 0 & 1
\end{array}\right)\left(\begin{array}{c}
\lambda_{12} \\
\lambda_{13} \\
\lambda_{14} \\
\lambda_{23} \\
\lambda_{24} \\
\lambda_{34}
\end{array}\right) \\
\left(\begin{array}{l}
\alpha_{2}\left(\lambda_{12}\right) \\
\alpha_{2}\left(\lambda_{13}\right) \\
\alpha_{2}\left(\lambda_{14}\right) \\
\alpha_{2}\left(\lambda_{23}\right) \\
\alpha_{2}\left(\lambda_{24}\right) \\
\alpha_{2}\left(\lambda_{34}\right)
\end{array}\right)=\left(\begin{array}{llllll}
0 & 0 & 0 & 0 & 0 & 1 \\
0 & 1 & 0 & 0 & 0 & 0 \\
0 & 0 & 0 & 1 & 0 & 0 \\
0 & 0 & 1 & 0 & 0 & 0 \\
0 & 0 & 0 & 0 & 1 & 0 \\
1 & 0 & 0 & 0 & 0 & 0
\end{array}\right)\left(\begin{array}{c}
\lambda_{12} \\
\lambda_{13} \\
\lambda_{14} \\
\lambda_{23} \\
\lambda_{24} \\
\lambda_{34}
\end{array}\right) .
\end{gathered}
$$

We now investigate the compatibility of the linear bijection $\Theta$ of theorem 4.2.6 with the symmetry group $\mathcal{T}_{64}$ of $X_{0}$ :

Proposition 4.3.4 Consider the square Kummer surface $X_{0}$ with its holomorphic symplectic automorphism group $\mathcal{T}_{64}$ according to proposition 4.3.3. Let $M_{\mathcal{T}_{64}}$ denote the sublattice of $H_{*}(X, \mathbb{Z})$ introduced in theorem 3.3 .7 , and let $i_{\mathcal{T}_{64}}: M_{\mathcal{T}_{64}} \longrightarrow N(-1)$ denote the restriction of the map $\Theta$ of theorem 4.2.6 to $M_{\mathcal{T}_{64}}$.

The map $i_{\mathcal{T}_{64}}$ is equivariant with respect to the symmetry group $\mathcal{T}_{64}$, where the action of $\iota_{1}, \ldots, \iota_{4}$ on $N$ is given by (4.1), and the generators $\alpha_{1}, \alpha_{2}$ act by

$$
\begin{aligned}
& \alpha_{1}=(4,8)(6,19)(10,20)(11,13)(12,22)(14,17)(16,18)(23,24), \\
& \alpha_{2}=(2,21)(3,9)(4,8)(10,12)(11,14)(13,17)(20,22)(23,24) .
\end{aligned}
$$

Proof: The equivariance of $i_{\mathcal{T}_{64}}$ with respect to $\iota_{1}, \ldots, \iota_{4}$ follows immediately from proposition 4.1.1. 
For the generators $\alpha_{1}, \alpha_{2}$ of $\mathcal{T}_{64}$, we find that the transformations (4.23) induce permutations $\left[\alpha_{k}\right]$ on the singular points of $T_{0} / \mathbb{Z}_{2}$ and thus on the elements of our hypercube $\mathbb{F}_{2}^{4}$. For the induced permutations $\widehat{\alpha}_{k}$ on $\mathcal{I} \backslash \mathcal{O}_{9}$, imposing equivariance of $i_{\mathcal{T}_{64}}$ amounts to the condition $\widehat{\alpha}_{k}(n)=\left[\alpha_{k}\right](I(n))$ for all $n \in \mathcal{I} \backslash \mathcal{O}_{9}$, where $I$ is the map (2.14). We obtain

$$
\begin{aligned}
& \widehat{\alpha}_{1}=(4,8)(10,20)(11,13)(12,22)(14,17)(16,18), \\
& \widehat{\alpha}_{2}=(2,21)(4,8)(10,12)(11,14)(13,17)(20,22) .
\end{aligned}
$$

From (4.24) we obtain the induced action of $\alpha_{1}$ on the $\pi_{*} \lambda_{i j}$, such that equivariance of (4.2) implies that the induced action of $\alpha_{1}$ on the $Q_{i j}$ must fix $Q_{12}$ and $Q_{34}$ and yield $Q_{13} \leftrightarrow Q_{24}, Q_{14} \leftrightarrow Q_{23}$. Given our choices for the $Q_{i j}$ of proposition 4.2.5 which lead to (4.17), this action amounts to the permutation $\tau_{1}:=(6,19)(23,24)$.

Similarly, (4.25) implies that $\alpha_{2}$ must fix $Q_{13}$ and $Q_{24}$ and must induce $Q_{12} \leftrightarrow Q_{34}, Q_{14} \leftrightarrow$ $Q_{23}$, which amounts to the permutation $\tau_{2}:=(3,9)(23,24)$. One now checks that the permutations $\alpha_{k}:=\widehat{\alpha}_{k} \circ \tau_{k}$, which agree with the permutations $\alpha_{1}, \alpha_{2}$ in our claim (4.26), are elements of the Mathieu group $M_{24}$.

We actually arrive at the surprising results of

Theorem 4.3.5 Consider the square Kummer surface $X_{0}$ of definition 4.3 .1 with its holomorphic symplectic automorphism group $\mathcal{T}_{64}$. Let $M_{\mathcal{T}_{64}}$ denote the sublattice of $H_{*}(X, \mathbb{Z})$ introduced in theorem 3.3.7, and let $i_{\mathcal{T}_{64}}$ denote the restriction of the map $\Theta$ of theorem 4.2.6 to $M_{\mathcal{T}_{64}}$.

The map $i_{\mathcal{T}_{64}}$ yields an isometric embedding $i_{\mathcal{T}_{64}}: M_{\mathcal{T}_{64}} \hookrightarrow N(-1)$ of the type constructed in proposition 4.1.1. Moreover, $i_{\mathcal{T}_{64}}$ is equivariant with respect to the group $\mathcal{T}_{64}$, where on $H_{*}(X, \mathbb{Z})$ the action of $\mathcal{T}_{64}$ is induced by its action on $X_{0}$ as holomorphic symplectic automorphism group, and on the Niemeier lattice $N$ of type $A_{1}^{24}$ its action is generated by $\iota_{1}, \ldots, \iota_{4}, \alpha_{1}, \alpha_{2}$ as in (4.1) and (4.26). The lattice $M_{\mathcal{T}_{64}}$ has rank 20.

Proof: By proposition 3.3.6, the lattice $M_{\mathcal{T}_{64}}$ is the orthogonal complement of the lattice $\pi_{*}\left(H_{2}\left(T_{0}, \mathbb{Z}\right)^{\mathcal{O}}\right) \oplus \operatorname{span}_{\mathbb{Z}}\left\{v_{0}+v\right\}$, where $\mathcal{O}$ is the binary dihedral group of order 8 acting as nontranslational holomorphic symplectic automorphism group of the square torus. By proposition 4.3.2 the lattice $\pi_{*}\left(H_{2}\left(T_{0}, \mathbb{Z}\right)^{\mathcal{O}}\right)$ is generated by $J_{1}:=\pi_{*} \lambda_{13}-\pi_{*} \lambda_{24}, J_{2}:=\pi_{*} \lambda_{14}+\pi_{*} \lambda_{23}, J_{3}:=$ $\pi_{*} \lambda_{12}+\pi_{*} \lambda_{34}$. Hence the lattice $M_{\mathcal{T}_{64}}$ consists of

$$
\operatorname{span}_{\mathbb{Z}}\left\{J_{1}^{\perp}, J_{2}^{\perp}, J_{3}^{\perp}\right\} \oplus \mathcal{P},
$$

with $J_{k}^{\perp}, k=1,2,3$ as in (4.21), along with the appropriate rational combinations of contributions from $K$ and $\Pi$ obtained by our gluing for generic Kummer surfaces. In particular, $M_{\mathcal{T}_{64}}$ has rank 20 .

We need to show that $\Theta$ maps this lattice isometrically to a primitive sublattice of $N(-1)$. We already know that $\Theta(\mathcal{P})=\widetilde{\mathcal{P}}_{5}(-1)$ isometrically, due to the proof of theorem 4.2.6. Since we also know that $\Theta$ is compatible with the gluing of $H_{*}(X, \mathbb{Z})$ and $N$ from $\mathcal{P}$ and $\widetilde{\mathcal{P}}_{5}$ and their orthogonal complements by that same proof, it suffices to show that the lattice generated by the $J_{k}^{\perp}, k \in\{1,2,3\}$, is isometric to the lattice generated by the three vectors $\Theta\left(J_{k}^{\perp}\right), k \in\{1,2,3\}$, up to an inversion of signature. In fact, note that by (4.16) we have

$$
J_{1}^{\perp} \longmapsto f_{24}-f_{23}, \quad J_{2}^{\perp} \longmapsto f_{19}-f_{6}, \quad J_{3}^{\perp} \longmapsto f_{9}-f_{3},
$$

yielding the quadratic form of the corresponding lattices with respect to these generators as

$$
\left(\begin{array}{rrr}
-4 & 0 & 0 \\
0 & -4 & 0 \\
0 & 0 & -4
\end{array}\right) \longmapsto\left(\begin{array}{lll}
4 & 0 & 0 \\
0 & 4 & 0 \\
0 & 0 & 4
\end{array}\right)
$$


on both sides, as required.

Finally, equivariance with respect to $\mathcal{T}_{64}$ now follows from proposition 4.3.4.

Summarising, we have confirmed the surprising fact that the same bijection $\Theta$ of theorem 4.2 .6 is compatible with the symmetry groups of two distinct Kummer surfaces, namely the tetrahedral Kummer surface and the square Kummer surface.

\subsection{Generating the overarching finite symmetry group of Kummer surfaces}

Let us now discuss the consequences of theorems 4.2.6 and 4.3.5, which state that our map $\Theta$ induces two isometric embeddings $M_{\mathcal{T}_{192}} \hookrightarrow N(-1)$ and $M_{\mathcal{T}_{64}} \hookrightarrow N(-1)$. We can view $\Theta$ as an OVERARCHING BIJECTION for these two embeddings. Theorems 4.2 .6 and 4.3 .5 also show that $\Theta$ is compatible with enforcing equivariance on each $i_{\mathcal{T}_{k}}, k \in\{64,192\}$, with respect to the symmetry group $\mathcal{T}_{k}$, inducing actions of these groups on the Niemeier lattice $N$ of type $A_{1}^{24}$ as subgroups of the Mathieu group $M_{24}$. Hence the lattice $N$ serves as a device which carries both these actions simultaneously, allowing us to make sense of combining these groups to a bigger group:

Theorem 4.4.1 Consider the subgroup $M_{\Pi}$ of the Mathieu group $M_{24}$ which is obtained by the combined actions of $\mathcal{T}_{192}$ and $\mathcal{T}_{64}$ of theorems 4.2.6 and 4.3.5 on the Niemeier lattice $N$ of type $A_{1}^{24}$. This group is isomorphic to the overarching finite symmetry group $\left(\mathbb{Z}_{2}\right)^{4} \rtimes A_{7}$ of Kummer surfaces of definition 3.3.5.

If $X$ is an arbitrary Kummer surface with induced dual Kähler class and holomorphic symplectic automorphism group $G$, let $i_{G}: M_{G} \hookrightarrow N(-1)$ denote an isometric embedding of the lattice $M_{G}$ of theorem 3.3 .7 according to proposition 4.1 .1 such that $i_{G}(\mathcal{P})=\widetilde{\mathcal{P}}_{n_{0}}(-1)$ with $n_{0} \in \mathcal{O}_{9}$. By enforcing $G$-equivariance on $i_{G}$, the group $G$ acts faithfully on the Niemeier lattice $N$. If $n_{0}=5$, then this realises $G$ as a subgroup of the group $M_{\Pi}$. If $n_{0} \neq 5$, then $G$ is a subgroup of a conjugate of $M_{\Pi}$ within the subgroup $\left(\mathbb{Z}_{2}\right)^{4} \rtimes A_{8}$ of $M_{24}$ which stabilizes the octad $\mathcal{O}_{9}$. In this sense, $\left(\mathbb{Z}_{2}\right)^{4} \rtimes A_{7}$ contains the holomorphic symplectic automorphism group of every Kummer surface with induced dual Kähler class.

Proof: By theorems 4.2.6 and 4.3.5, the group $M_{\Pi}$ is generated by $\iota_{1}, \ldots, \iota_{4}, \gamma_{1}, \gamma_{2}, \gamma_{3}$ as in (4.13) and $\alpha_{1}, \alpha_{2}$ as in (4.26). Clearly, the permutations $\iota_{1}, \ldots, \iota_{4}$ generate a faithful action of $\left(\mathbb{Z}_{2}\right)^{4}$ on $\mathcal{I}=\{1, \ldots, 24\}$ which leaves the labels in the special octad $\mathcal{O}_{9}$ invariant pointwise. Furthermore, one readily sees that $\gamma_{1}, \gamma_{2}, \gamma_{3}, \alpha_{1}, \alpha_{2}$ induce a faithful action of $A_{7}$ on $\mathcal{O}_{9} \backslash\{5\}$. Hence $M_{\Pi} \supset\left(\mathbb{Z}_{2}\right)^{4} \rtimes A_{7}$.

On the other hand, by construction both our groups $\mathcal{T}_{192}$ and $\mathcal{T}_{64}$ act on $N$ as subgroups of the stabilizer subgroup of $M_{24}$ of our special octad $\mathcal{O}_{9}$. According to 28 this group is a maximal subgroup of $M_{24}$, and it is isomorphic to $\left(\mathbb{Z}_{2}\right)^{4} \rtimes A_{8}$, where $A_{8}$ is realised as the group of permutations undergone by the 8 points of $\mathcal{O}_{9}$. Since in addition, both our groups $\mathcal{T}_{192}$ and $\mathcal{T}_{64}$ stabilize the label $5=n_{0} \in \mathcal{O}_{9}$, we find that both of them are subgroups of the corresponding maximal subgroup $\left(\mathbb{Z}_{2}\right)^{4} \rtimes A_{7}$ of $M_{23}$. In particular, $M_{\Pi} \subset\left(\mathbb{Z}_{2}\right)^{4} \rtimes A_{7}$, thus by the above $M_{\Pi} \cong\left(\mathbb{Z}_{2}\right)^{4} \rtimes A_{7}$ is isomorphic to the overarching finite symmetry group of Kummer surfaces, as claimed.

Consider a Kummer surface $X$ with induced dual Kähler class and holomorphic symplectic automorphism group $G$, and the corresponding lattice $M_{G}$ that was constructed in theorem 3.3.7. Let $i_{G}: M_{G} \hookrightarrow N(-1)$ denote the isometric embedding with $i_{G}(\mathcal{P})=\widetilde{\mathcal{P}}_{n_{0}}(-1)$ according to proposition 4.1.1, where $n_{0} \in \mathcal{O}_{9}$. Assume first that $n_{0}=5$. Then the action of $G$ on $N$ obtained by enforcing $G$-equivariance on $i_{G}$ maps $\widetilde{\mathcal{K}}_{5}=\widetilde{\mathcal{P}}_{5}^{\perp} \cap N$ onto itself. By the proof of proposition 2.3.6 we know that $\widetilde{\mathcal{K}}_{5}=\operatorname{span}_{\mathbb{Z}}\left\{f_{3}, f_{6}, f_{9}, f_{15}, f_{19}, f_{23}, f_{24}\right\}$. Hence $G$ stabilizes the special octad $\mathcal{O}_{9}=\{3,5,6,9,15,19,23,24\}$ and fixes $n_{0}=5$. Thus, by the 
above, $G$ is a subgroup of the maximal subgroup $M_{\Pi} \cong\left(\mathbb{Z}_{2}\right)^{4} \rtimes A_{7}$ of $M_{23}$. If $n_{0} \neq 5$, then analogously one finds $G \subset M_{\Pi}^{\prime} \cong\left(\mathbb{Z}_{2}\right)^{4} \rtimes A_{7}$ where $M_{\Pi}^{\prime}$ is the subgroup of $M_{24}$ containing those automorphisms that stabilize the special octad $\mathcal{O}_{9}$ and that fix $n_{0}$. Using the structure of the stabilizer group of $\mathcal{O}_{9}$ described above, one finds that $M_{\Pi}^{\prime}$ is obtained from $M_{\Pi}$ by conjugation within the subgroup $\left(\mathbb{Z}_{2}\right)^{4} \rtimes A_{8}$ of $M_{24}$ which stabilizes the octad $\mathcal{O}_{9}$.

The group $M_{\Pi} \cong\left(\mathbb{Z}_{2}\right)^{4} \rtimes A_{7}$ obtained in theorem 4.4.1 has order 40320. By the results of the theorem it is also the largest group we can possibly obtain by combining several holomorphic symplectic automorphism groups of Kummer surfaces through equivariant embeddings overarched by some linear bijection $H_{*}(X, \mathbb{Z}) \longrightarrow N(-1)$ like our map $\Theta$ of theorem 4.2.6.

The construction of an overarching map $\Theta: H_{*}(X, \mathbb{Z}) \longrightarrow N(-1)$ for the two primitive embeddings $i_{G_{k}}: M_{\mathcal{T}_{k}} \hookrightarrow N(-1)$ makes sense, since we are choosing a fixed marking to identify $H_{*}(X, \mathbb{Z}), H_{2}(X, \mathbb{Z})$ with standard unimodular lattices of signatures $(4,20)$, $(3,19)$, which is induced by the Kummer construction, as was explained at the end of section 2.2. According to our remarks at the end of section 3.1, this marking allows us to construct Kummer paths in the smooth connected cover $\widetilde{\mathcal{M}}_{h k}$ of the moduli space of hyperkähler structures. Recall that each hyperkähler structure is represented by the 3-dimensional subspace $\Sigma \subset H_{2}(X, \mathbb{R})=H_{2}(X, \mathbb{Z}) \otimes \mathbb{R}$ generated by the 2-dimensional $\Omega \subset H_{2}(X, \mathbb{R})$, which specifies the complex structure according to the Torelli Theorem 3.1.1 and the dual Kähler class $\omega$. Along Kummer paths, each complex structure and (degenerate) dual Kähler class is induced from the universal cover $\mathbb{C}^{2}$ of an underlying torus $T(\Lambda)=\mathbb{C}^{2} / \Lambda, \Lambda=\operatorname{span}_{\mathbb{Z}}\left\{\vec{\lambda}_{1}, \ldots, \vec{\lambda}_{4}\right\}$, for a Kummer surface $T \widetilde{T(\Lambda) / \mathbb{Z}_{2}}$. Smooth variation of the generators $\vec{\lambda}_{1}, \ldots, \vec{\lambda}_{4}$ of the underlying lattice $\Lambda \subset \mathbb{C}^{2}$ yields a smooth variation between the hyperkähler structures of any two Kummer surfaces. Our marking allows us to view the lattice $H_{*}(X, \mathbb{Z})$ (with generators $\left.v_{0}, v ; \quad \frac{1}{2} \pi_{*} \lambda_{i j}+\frac{1}{2} \sum_{\vec{a} \in P_{i j}} \varepsilon_{\vec{a}} E_{\vec{a}+\vec{b}}, i j \in\{12,34,13,42,14,23\}, \vec{b} \in \mathbb{F}_{2}^{4}, \varepsilon_{\vec{a}} \in\{ \pm 1\}\right)$ as fixed, while the 3 -dimensional space $\Sigma$ generated by $e_{1} \vee e_{3}-e_{2} \vee e_{4}, e_{1} \vee e_{4}+e_{2} \vee e_{3}, e_{1} \vee e_{2}+e_{3} \vee e_{4}$ (see (3.2), (3.5)) varies, since the expressions of each $e_{i}$ in terms of $\vec{\lambda}_{1}, \ldots, \vec{\lambda}_{4}$ vary with $\Lambda$. In view of this remark it is natural to ask whether our overarching map $\Theta$ is compatible with the embeddings $i_{G^{s}}: M_{G^{s}} \hookrightarrow N(-1)$ of proposition 4.1.1 along some Kummer path connecting the tetrahedral and the square Kummer surface. Indeed, this is the case:

Theorem 4.4.2 There exists a smooth path in the smooth connected cover $\widetilde{\mathcal{M}}_{h k}$ of the moduli space of hyperkähler structures of $\mathrm{K} 3$ with the following properties:

Let $\Sigma_{s}$ denote the positive definite oriented 3-dimensional subspace of $H_{2}(X, \mathbb{R})$ generated by the 2-dimensional $\Omega_{s}$, which specifies the complex structure according to the Torelli Theorem 3.1.1, and $\omega_{s}$, the dual Kähler class, at time $s \in[0,1]$ along the path. These data are specified by their relative positions with respect to the lattice $H_{2}(X, \mathbb{Z})$ which is given in terms of the even unimodular lattice of signature $(3,19)$ arising from the Kummer construction, as explained at the end of section [2.2. Then for each $s \in[0,1]$ :

- $\Omega_{s}, \omega_{s}$ give the complex structure and (degenerate) dual Kähler class of a Kummer surface $T \widetilde{\left(\Lambda_{s}\right) / \mathbb{Z}_{2}}$ and are induced from the standard structures on $\mathbb{C}^{2}$, the universal cover of $T\left(\Lambda_{s}\right)=\mathbb{C}^{2} / \Lambda_{s}$.

- $\Omega_{0}, \omega_{0}$ give the complex structure and (degenerate) dual Kähler class of the square Kummer surface $X_{0}$.

- $\Omega_{1}, \omega_{1}$ give the complex structure and (degenerate) dual Kähler class of the tetrahedral Kummer surface $X_{D_{4}}$.

- Let $G^{s}$ denote the symmetry group of the Kummer surface with data $\Omega_{s}, \omega_{s}$ and $M_{G^{s}} \subset$ $H_{*}(X, \mathbb{Z})$ the lattice of theorem 3.3.7. Then for the map $\Theta: H_{*}(X, \mathbb{Z}) \longrightarrow N(-1)$ constructed in theorem 4.2.6, $i_{G^{s}}:=\Theta_{\mid M_{G^{s}}}: M_{G^{s}} \hookrightarrow N(-1)$ is an isometric, primitive 
embedding. Enforcing $G^{s}$-equivariance, $G^{s}$ acts as subgroup of the overarching symmetry group $M_{\Pi} \cong\left(\mathbb{Z}_{2}\right)^{4} \rtimes A_{7} \subset M_{24}$ on $N$ which was found in theorem 4.4.1.

In particular, $s \mapsto \Sigma_{s}, s \in[0,1]$ describes a Kummer path along which $i_{G}(\Pi)=\widetilde{\Pi}$ is constant.

Proof: We show that there exists a Kummer path $s \mapsto \Sigma_{s}, s \in[0,1]$, in $\widetilde{\mathcal{M}}_{h k}$ which connects the square Kummer surface $X_{0}$ with the tetrahedral Kummer surface $X_{D_{4}}$ such that for every $s \in(0,1)$, the only holomorphic symplectic automorphisms are the translational ones, i.e. $G^{s} \cong\left(\mathbb{Z}_{2}\right)^{4}, M_{G^{s}}=\Pi \oplus \operatorname{span}_{\mathbb{Z}}\left\{v_{0}-v\right\}$. Then the claims immediately follow by construction, since compatibility with the translational group $G^{s}=G_{t} \cong\left(\mathbb{Z}_{2}\right)^{4}$ is incorporated at the beginning of our construction of $\Theta$ in section 4.1.

Since the desired path is a Kummer path, we must ensure $\Sigma_{s} \subset \pi_{*} H_{2}(T, \mathbb{R})$ for all $s \in$ $[0,1]$ with the notations of section 2.2. Proposition 3.3 .4 implies that $G^{s}=G_{t} \cong\left(\mathbb{Z}_{2}\right)^{4}$ if $\left(H_{2}(X, \mathbb{Z})^{G^{s}}\right)^{\perp} \cap H_{2}(X, \mathbb{Z})=\Pi$ or equivalently $\left(H_{2}(X, \mathbb{Z})^{G^{s}}\right)^{\perp} \cap \pi_{*} H_{2}(T, \mathbb{Z})=\{0\}$. In general, $\Sigma_{s} \subset H_{2}(X, \mathbb{Z})^{G^{s}} \otimes \mathbb{R}$ and thus $\left(\Sigma_{s}\right)^{\perp} \supset\left(H_{2}(X, \mathbb{Z})^{G^{s}}\right)^{\perp}$; hence it suffices to ensure $\left(\Sigma_{s}\right)^{\perp} \cap \pi_{*} H_{2}(T, \mathbb{Z})=\{0\}$ for all $s \in(0,1)$. Using the $I_{k}^{\perp}$ and $J_{k}^{\perp}$ of (4.9) and (4.21) we set $\Sigma_{T, s}^{\perp}:=\operatorname{span}_{\mathbb{R}}\left\{I_{1, s}^{\perp}, I_{2, s}^{\perp}, I_{3, s}^{\perp}\right\} \subset \pi_{*} H_{2}(T, \mathbb{R})$,

$$
I_{k, s}^{\perp}:= \begin{cases}(1-s) J_{k}^{\perp}+s I_{k}^{\perp}+2 s \delta_{k} \cdot \pi_{*} \lambda_{12} & \text { if } s \in\left[0, \frac{1}{2}\right], \\ (1-s) J_{k}^{\perp}+s I_{k}^{\perp}+2(1-s) \delta_{k} \cdot \pi_{*} \lambda_{12} & \text { if } s \in\left[\frac{1}{2}, 1\right],\end{cases}
$$

with sufficiently small $\delta_{1}, \delta_{2}, \delta_{3} \in \mathbb{R}$, such that $\Sigma_{T, s}^{\perp}$ is negative definite, which are linearly independent over $\mathbb{Q}$. One checks that $I_{k, 0}^{\perp}=J_{k}^{\perp}$ and $I_{k, 1}^{\perp}=I_{k}^{\perp}$ for $k \in\{1,2,3\}$, and $\Sigma_{T, s}^{\perp} \cap \pi_{*} H_{2}(T, \mathbb{Z})=\{0\}$ for all $s \in(0,1)$. Hence $\Sigma_{s}:=\left(\Sigma_{T, s}^{\perp}\right)^{\perp} \cap \pi_{*} H_{2}(T, \mathbb{R})$ defines a Kummer path from the square Kummer surface to the tetrahedral Kummer surface with the desired properties.

Although we have not discussed the detailed proof of this statement here, it is worthwhile mentioning that our map $\Theta$ is almost uniquely determined by the requirement that it induces isometric embeddings of the type constructed in proposition 4.1.1 for both lattices $M_{\mathcal{T}_{192}}$ and $M_{\mathcal{T}_{64}}$. Our explicit formulas, of course, first of all depend on our choice of $\mathcal{O}_{9}$ as our special octad, and more precisely on the particular embedding of the Kummer lattice $\Pi$ in the Niemeier lattice $N(-1)$ of type $A_{1}^{24}$ that we constructed in the proof of proposition 2.3.4. However, by proposition 2.3.5 any other embedding of $\Pi$ is related to the one used by us by an automorphism of the Niemeier lattice $N$. On the level of subgroups of the Mathieu group $M_{24}$, this amounts to conjugating by some element of $M_{24}$.

Once our embedding of $\Pi$ in $N(-1)$ has been chosen, our requirements on $\Theta$ turn out to enforce the choices (4.14) for the quadruplets $Q_{i j}$ in our special octad $\mathcal{O}_{9}$. Concerning the choices of signs in (4.2), as mentioned in the discussion of equation (4.17), these are unique up to the freedom for each $n \in \mathcal{I}$ to replace $f_{n}$ by $-f_{n}$ everywhere, and up to some choices of signs in the image of $v_{0}+v$.

Now recall once again that the involutions on the Niemeier lattice $N$ of type $A_{1}^{24}$ induced by $f_{n} \mapsto-f_{n}$ should not play a role in describing symmetries of Kummer surfaces, or in fact their associated superconformal field theories: On the one hand, by proposition 2.3.2 the Mathieu group $M_{24}$, which we are interested in, is obtained as factor group of $\operatorname{Aut}(N)$ by the normal subgroup $\left(\mathbb{Z}_{2}\right)^{24}$ generated by these involutions. On the other hand, consider a symplectic automorphism of a $\mathrm{K} 3$ surface $X$ whose induced action on $H_{*}(X, \mathbb{Z})$ is given by the lattice automorphism $\alpha$. We claim that $\alpha(E) \neq-E$ for every $E \in H_{*}(X, \mathbb{Z})$ with $\langle E, E\rangle=-2$. Indeed, $\alpha(v)=-v$ for some $v \in H_{*}(X, \mathbb{Z})$ immediately implies $v \perp L_{\bullet}^{\alpha}$, where $L_{\bullet}^{\alpha}:=H_{*}(X, \mathbb{Z})^{\alpha}$ is the sublattice of $H_{*}(X, \mathbb{Z})$ which is invariant under $\alpha$. Since 
$L_{\bullet}^{\alpha}=H_{0}(X, \mathbb{Z}) \oplus L^{\alpha} \oplus H_{4}(X, \mathbb{Z})$ with $L^{\alpha}:=H_{2}(X, \mathbb{Z})^{\alpha}$, this implies that $v \in L_{\alpha}:=\left(L_{\bullet}^{\alpha}\right)^{\perp} \cap$ $H_{*}(X, \mathbb{Z})=\left(L^{\alpha}\right)^{\perp} \cap H_{2}(X, \mathbb{Z})$. Hence by the very Torelli Theorem 3.2.2, $\langle v, v\rangle \neq-2$. By arguments analogous to those used for symplectic automorphisms of K3 surfaces, one may deduce the following from the definition of SYMMETRIES OF NON-LINEAR $\sigma$-MODELS ON K3 PRESERVing the $\mathcal{N}=(4,4)$ SUPERCONFormal algebra given in [15]: Firstly, any such symmetry induces a lattice automorphism $\alpha$ on $H_{*}(X, \mathbb{Z})$, which in turn uniquely determines the symmetry. Furthermore, $\alpha(E) \neq-E$ for every $E \in H_{*}(X, \mathbb{Z})$ with $\langle E, E\rangle=-2$. In particular, an involution of the Niemeier lattice $N$ induced by $f_{n} \mapsto-f_{n}$ can never correspond to a symplectic automorphism of a K3 surface or to a symmetry of a non-linear $\sigma$-model on $\mathrm{K} 3$ which preserves the $\mathcal{N}=(4,4)$ superconformal algebra.

\section{Conclusions and outlook}

This work gives a novel perspective on holomorphic symplectic automorphisms of Kummer surfaces whose dual Kähler class is induced by the underlying complex torus. While finite symplectic automorphism groups of K3 surfaces, in general, have been classified and described by Mukai and Kondo in their seminal works [9, 10], we improve their description in the case of Kummer surfaces through an identification of lattice automorphisms. This provides a concrete representation in terms of permutations of 24 elements which are symmetries of the binary extended Golay code, and thus in terms of subgroups of the Mathieu group $M_{24}$.

For a K3 surface $X$ with holomorphic symplectic automorphism group $G$ and $L_{G} \subset H_{*}(X, \mathbb{Z})$ the orthogonal complement of the $G$-invariant part of the K3-homology, one finds by the results of Kondo [10] a Niemeier lattice $\widetilde{N}$, which carries a faithful $G$-action, such that $L_{G}$ can be primitively and $G$-equivariantly embedded in $\widetilde{N}(-1)$. We improve this result for all Kummer surfaces whose dual Kähler class is induced from the underlying complex torus: We prove that in this case the Niemeier lattice $\widetilde{N}$, which is not further specified in Kondo's construction, can be replaced by the Niemeier lattice $N$ of type $A_{1}^{24}$. Moreover, the lattice $L_{G}$ can be replaced by a lattice $M_{G}$ with $\operatorname{rk}\left(M_{G}\right)=\operatorname{rk}\left(L_{G}\right)+2$ which contains $L_{G}$, that is, $M_{G}$ can be primitively and $G$-equivariantly embedded in $N(-1)$. We deduce that for every Kummer surface whose dual Kähler class is induced from the underlying torus, one can construct a $\mathbb{Z}$-linear bijection $\theta: H_{*}(X, \mathbb{Z}) \longrightarrow N(-1)$ whose restriction to $M_{G}$ yields an isometric, $G$-equivariant primitive embedding.

We explicitly construct a map $\Theta$ with the above-mentioned properties for the tetrahedral Kummer surface $X_{D_{4}}$ with holomorphic symplectic automorphism group $\mathcal{T}_{192}$ of order 192. The nature of this map depends on the details of the complex structure and dual Kähler class, and its analog for other Kummer surfaces must therefore be constructed case by case, but is not difficult to obtain in the framework set up in our work.

Our construction uses a description of the lattice $H_{*}(X, \mathbb{Z})$ by means of lattice generators naturally arising in the Kummer construction. This provides us with a common, fixed marking for all Kummer surfaces. Varying the generators $\vec{\lambda}_{1}, \ldots, \vec{\lambda}_{4}$ of the defining lattice of the underlying torus then amounts to a deformation along a path in the smooth connected cover of the moduli space of hyperkähler structures on K3. In particular, having fixed a common, natural marking for all Kummer surfaces allows us to simultaneously study distinct Kummer surfaces. Surprisingly, our map $\Theta$, apart from restricting to an isometric, $\mathcal{T}_{192}$-equivariant embedding of $M_{\mathcal{T}_{192}}$ in $N(-1)$, also restricts to an isometric, $G$-equivariant embedding of $M_{G}$ in $N(-1)$ for a different Kummer surface $X_{0}$, namely the one obtained from the square torus $T_{0}$. The holomorphic symplectic symmetry group $G=\mathcal{T}_{64}$ of $X_{0}$ has order 64 . We find a smooth path in the smooth connected cover of the moduli space of hyperkähler structures on $\mathrm{K} 3$ between these two Kummer surfaces, such that $\Theta$ is compatible with the symmetries of all Kummer surfaces along the path. The latter have the translational group $G_{t} \cong\left(\mathbb{Z}_{2}\right)^{4}$ 
as symmetry group, except for the Kummer surfaces at the endpoints, which have symmetry groups $\mathcal{T}_{64}$ and $\mathcal{T}_{192}$ as explained above. The translational group $G_{t} \cong\left(\mathbb{Z}_{2}\right)^{4}$, which is compatible with $\Theta$ by construction, is the symmetry group of generic Kummer surfaces.

By construction, the lattice $M_{G}$ is negative definite for every Kummer surface. Both for $X_{D_{4}}$ and for $X_{0}$ it turns out to have the maximal possible rank 20. The smallest primitive sublattice of $H_{*}(X, \mathbb{Z})$ containing both lattices $M_{\mathcal{T}_{192}}$ and $M_{\mathcal{T}_{64}}$ is $H_{2}(X, \mathbb{Z}) \oplus \operatorname{span}_{\mathbb{Z}}\left\{v_{0}-v\right\}$, where $v_{0} \in H_{0}(X, \mathbb{Z})$ and $v \in H_{4}(X, \mathbb{Z})$ with $\left\langle v_{0}, v\right\rangle=1$.

Our map $\Theta$ thus yields an action of the groups $\mathcal{T}_{192}$ and $\mathcal{T}_{64}$ on the Niemeier lattice $N$, realising each of them as a subgroup of the Mathieu group $M_{24}$. Again, the nature of the respective permutations depends on the details of the complex structure and dual Kähler class, and their analog for other Kummer surfaces can be worked out similarly. There are further Kummer surfaces whose symmetries are consistently described by our specific map $\Theta$. For example, the $\mathbb{Z}_{3}$-symmetric torus $T_{(3)}=\mathbb{C}^{2} / \Lambda_{(3)}$ with $\Lambda_{(3)}$ generated by

$$
\vec{\lambda}_{1}=(1,0), \quad \vec{\lambda}_{2}=(i, 0), \quad \vec{\lambda}_{3}=\left(\frac{1}{2}, \frac{\sqrt{3}}{2}\right), \quad \vec{\lambda}_{4}=\left(-\frac{i}{2}, \frac{i \sqrt{3}}{2}\right) \quad \in \mathbb{C}^{2}
$$

yields a Kummer surface whose holomorphic symplectic automorphism group $G$ is also compatible with our map $\Theta$, if for the two-cycles yielding complex structure and polarization one uses

$$
\widetilde{\Omega}_{1}=e_{1} \vee e_{2}+e_{3} \vee e_{4}, \quad \widetilde{\Omega}_{2}=-e_{1} \vee e_{4}-e_{2} \vee e_{3}, \quad \widetilde{\omega}=e_{1} \vee e_{3}-e_{2} \vee e_{4}
$$

In this case our map $\Theta$ restricts to a primitive $G$-equivariant map from $M_{G}$ to $N(-1)$, which however is not isometric.

While $\Theta$ is by no means compatible with all the symmetries of all Kummer surfaces, the surprising observation is the fact that it yields a device which allows to simultaneously realise several finite symplectic automorphism groups of different Kummer surfaces in terms of subgroups of $M_{23}$ acting on $N$. The combined action of these groups yields the overarching finite symmetry group $\left(\mathbb{Z}_{2}\right)^{4} \rtimes A_{7}$ of Kummer surfaces, which contains every holomorphic symplectic automorphism group of a Kummer surface with induced dual Kähler class as a subgroup, and it is the maximal group that our techniques can describe, so far.

Although we do not present the proof in this work, we remark that for the above-mentioned $\mathbb{Z}_{3}$-symmetric torus $T_{(3)}$ we can show that no isometric embedding $M_{G} \hookrightarrow N(-1)$ exists which maps $v_{0}-v$ to $f_{5}=\Theta\left(v_{0}-v\right)$. It follows that along a path from $X_{0}$, say, to $\widetilde{T_{(3)} / \mathbb{Z}_{2}}$, starting with the embedding $M_{G_{0}} \hookrightarrow N(-1)$ constructed in this work, $v_{0}-v$ must change its image. This probably implies that an extension of our techniques to more general paths in moduli space will yield subgroups of $M_{24}$ rather than $M_{23}$, as will be explained in [51].

A crucial observation which underlies our construction is the fact that the Niemeier lattice $N$ of type $A_{1}^{24}$ contains a primitive sublattice $\widetilde{\Pi}$ which is isometric to the Kummer lattice $\Pi$, up to a total inversion of signature. This observation - though not very hard to prove - is new, and it gives a geometric meaning to a fact known to group theorists, namely that the complement of an octad in the extended binary Golay code naturally carries the structure of a 4-dimensional vector space over $\mathbb{F}_{2}$. In fact, the Kummer lattice $\Pi$ is a primitive sublattice of our lattice $M_{G}$ for every Kummer surface, while $\Pi$ can never be a sublattice of the lattice $L_{G} \subset M_{G}$ used by Kondo in his construction. Our lattice $M_{G}$ can be generated by Kondo's lattice $L_{G}$ together with the Kummer lattice $\Pi$ and the vector $v_{0}-v$ mentioned above. This vector gives a geometric interpretation to the invariant root needed in Kondo's construction, whose role had been mysterious, so far.

The lattices $\Pi$ and $\widetilde{\Pi}$, however, govern the symmetries in our holomorphic symplectic automorphism groups $G$, as we shall explain now. By the known structure of $\Pi$ and $\widetilde{\Pi}$, the group of isometries for both lattices is isomorphic to $\operatorname{Aff}\left(\mathbb{F}_{2}^{4}\right) \ltimes\left(\mathbb{Z}_{2}\right)^{16}$, where each factor of $\left(\mathbb{Z}_{2}\right)^{16}$ is induced by inverting the sign of one lattice vector on which the quadratic form takes value 
\pm 2 . Hence restricting to the analogs of EFFECTIVE AUTOMORPHisMs known from geometry, we can restrict our attention to the affine linear maps in $\operatorname{Aff}\left(\mathbb{F}_{2}^{4}\right)=\left(\mathbb{Z}_{2}\right)^{4} \rtimes \operatorname{GL}\left(4, \mathbb{F}_{2}\right)$.

Consider the action of a lattice automorphism $\alpha$ on $\Pi \cong \widetilde{\Pi}(-1)$ with $\alpha \in \operatorname{Aff}\left(\mathbb{F}_{2}^{4}\right)$. If $\alpha$ is an element of the normal subgroup $\left(\mathbb{Z}_{2}\right)^{4}$, then it corresponds to a translation on the hypercube underlying the lattices $\Pi$ and $\widetilde{\Pi}$, and it induces a trivial action on the discriminant groups $\Pi^{*} / \Pi$ and $\widetilde{\Pi}^{*} / \widetilde{\Pi}$. Thus $\alpha$ can be trivially extended to a lattice automorphism of the K3homology $H_{*}(X, \mathbb{Z})$ and of the Niemeier lattice $N$ of type $A_{1}^{24}$. We claim that extensions to lattice automorphisms of $H_{*}(X, \mathbb{Z})$ and $N$ exist for every $\alpha \in \operatorname{Aff}\left(\mathbb{F}_{2}^{4}\right)$ which is represented by an action as automorphism of $\Pi \cong \widetilde{\Pi}(-1)$. For the K3-homology $H_{*}(X, \mathbb{Z})$ this follows immediately from [11, Thm. 1.7] (using [11, Remark 1.2]), since $\Pi^{\perp} \cap H_{*}(X, \mathbb{Z}) \cong U^{3}(2) \oplus U$ is an even lattice of signature $(4,4)$ whose discriminant group has length $6=4+4-2$. For the Niemeier lattice $N$ it is a consequence of the fact that the stabilizer group of an octad in the Golay code is isomorphic to $\left(\mathbb{Z}_{2}\right)^{4} \rtimes A_{8}$, where $A_{8}$ is realised as the group of permutations undergone by the 8 points of that octad according to [28]. Indeed, Conway's proof of [29, Thm. 2.10] shows that the induced action on $\widetilde{\Pi}$ is precisely our group of effective lattice automorphisms $\operatorname{Aff}\left(\mathbb{F}_{2}^{4}\right) \cong\left(\mathbb{Z}_{2}\right)^{4} \rtimes A_{8}$. Hence every $\alpha \in \operatorname{Aff}\left(\mathbb{F}_{2}^{4}\right) \subset \operatorname{Aut}(\widetilde{\Pi})$ is the restriction of some lattice automorphism of $N$ to the sublattice $\widetilde{\Pi}$, which in fact is uniquely determined. In the case of extending $\alpha$ to the full K3-homology, we do not have a uniqueness statement in general. However, restricting to lattice automorphisms which are induced by some holomorphic symplectic automorphism of a K3 surface, uniqueness follows from the Torelli Theorem.

In summary, the lattice automorphisms that are of interest in this work are completely determined by their restrictions to $\Pi$ and $\widetilde{\Pi}$, respectively. This explains why extending Kondo's lattice $L_{G}$ to a lattice $M_{G}$ which contains $\Pi$ is such a useful idea. That this idea works is one of our main results. It in particular implies that a bijection $\theta$ between $H_{*}(X, \mathbb{Z})$ and $N(-1)$ can be constructed which is compatible with the symmetry group $G_{t} \cong\left(\mathbb{Z}_{2}\right)^{4}$ of generic Kummer surfaces.

While it thus may be tempting to expect some natural geometric interpretation of the group $\left(\mathbb{Z}_{2}\right)^{4} \rtimes A_{8}$ in terms of holomorphic symplectic automorphisms of Kummer surfaces, we emphasize again that the overarching symmetry group $\left(\mathbb{Z}_{2}\right)^{4} \rtimes A_{7}$ is the largest group which can be induced by a map $\Theta$ obeying all our assumptions. As mentioned above, for other pairs of Kummer surfaces one will be forced to drop the assumption that an overarching map fixes the images of $v_{0}-v$ in $N(-1)$. Then one may be able to generate $\left(\mathbb{Z}_{2}\right)^{4} \rtimes A_{8}$ [51].

It should be noted that our map $\Theta$ is an isometry only when restricted to the appropriate lattices $M_{G}$. Equivalently, it need not be $G$-equivariant outside of $M_{G}$. The virtue of the specific map $\Theta$ which we construct in this work is therefore its compatibility with two distinct Kummer surfaces, namely the tetrahedral one and the one obtained from the square torus, and with all the Kummer surfaces along a special path connecting the two. This property defines $\Theta$ uniquely, up to a few choices of signs discussed in section 4.4, and only this property allows us to combine the actions of the symmetry groups $\mathcal{T}_{192}$ and $\mathcal{T}_{64}$ of the two Kummer surfaces to obtain the overarching symmetry group $\left(\mathbb{Z}_{2}\right)^{4} \rtimes A_{7}$.

This group has 40320 elements and thus is by orders of magnitude larger than the biggest finite symplectic automorphism group of any K3 surface. Our techniques thus mitigate the "order of magnitude" problem that was mentioned in the introduction. We are currently generalising our techniques by replacing the Niemeier lattice $N$ by the Leech lattice. This way we hope to obtain the action of the entire Mathieu group $M_{23}$ or $M_{24}$ on the Leech lattice merely using classical symmetries of K3 surfaces. This may mean that "Mathieu moonshine" has nothing to do with symmetries beyond the classical ones. 


\section{Acknowledgements}

We wish to thank Robert Curtis for sharing with us important insights on $M_{24}$ and infecting us with the MOG virus. We also benefitted from conversations with Geoffrey Mason and Michael Tuite, and many participants of the workshop on 'Mathieu Moonshine' at ETH Zurich in July 2011, including its organiser, Matthias Gaberdiel, whom we wish to thank for such an inspiring meeting. Matthias Schütt deserves our gratitude for a number of helpful comments. Moreover, we are grateful to a referee for a correction in the proof of Proposition 2.3.3 and comments leading to the Remark 3.2.3. We thank the London Mathematical Society for a Scheme 4 Collaborative Small Grant. AT is supported in part by a Leverhulme Research Fellowship RF/2012-335. She thanks Augsburg and Freiburg Universities for their hospitality. KW is supported in part by ERC-StG grant No. 204757-TQFT. She thanks Durham University for its hospitality. We furthermore thank the Hausdorff Research Institute of Mathematics and the Max-Planck Institute for Mathematics in Bonn for hospitality - a major part of the final version of this work was completed at Bonn.

The free open-source mathematics software system SAGE14, The Sage Development Team, 2010, http://www . sagemath.org and in particular its component GAP4 15 were used in checking several assertions made in the group theoretical component of our work.

\section{A The Mathieu group $M_{24}$, the binary Golay code and the MOG}

The relation between Kummer lattices and the group $M_{24}$ can be made explicit by thinking of $M_{24}$ as the proper subgroup of $A_{24}$ - the group of even permutations of 24 objects labelled by the elements of $\mathcal{I}=\{1,2,3, \ldots, 24\}$ - that preserves the extended binary Golay code $\mathcal{G}_{24}$. The latter is the dimension 12 quadratic residue code of length 23 over the field $\mathbb{F}_{2}$, extended in such a way that each element is augmented by a zero-sum check digit as described in [25]. The vector space $\mathcal{G}_{24}$ contains $2^{12}$ vectors called CODEWORDS, each being an element of $\mathbb{F}_{2}^{24}$, with the restrictions that their WEIGHT (the number of non-zero entries) is a multiple of 4 , bar 4 itself and 20. The code contains exactly one codeword zero and one codeword where all digits are one, together with 759 octads, 2576 dodecads and 759 complement octads.

Besides its description as a vector of $\mathbb{F}_{2}^{24}$ with components $c_{k}, k=1, \ldots, 24$, a non-zero codeword is often represented in the main text by a subset of $\mathcal{I}$ of cardinality 8, 12, 16 or 24 , whose elements are the integers $k$ labelling $c_{k} \neq 0$. So for instance, the word $(1,1,1,0,1,0,0,0,0,0,0,1,0,0,0,1,0,1,0,0,0,1,0,0)$ may be represented by the set $\{1,2,3,5,12,16,18,22\}$. That such a collection of eight labels is actually a weight 8 codeword of the binary Golay code can be readily checked by using an extremely powerful (and playful!) technique devised by Robert Curtis in the course of his extensive study of $M_{24}$ [52] and that we refer to as 'mogging', as it uses the Miracle Octad Generator (MOG). We have used a variant of the original technique, which was developed by Conway shortly after, and combines the hexacode $\mathcal{H}_{6}$ with the Miracle Octad Generator (MOG). These tools are well-documented in the literature (see 25] for instance), and we therefore confine ourselves to the bare essentials. The hexacode $\mathcal{H}_{6}$ is a 3 -dimensional code of length 6 over the field of four element $\mathbb{F}_{4}=$ $\left\{0,1, \omega, \omega^{2}\right\}$, with $\omega^{3}=1,1+\omega=\omega^{2}$, and $\bar{\omega}:=\omega^{2}$. It may be defined as

$$
\mathcal{H}_{6}=\left\{(a, b, \phi(0), \phi(1), \phi(\omega), \phi(\bar{\omega})) \mid a, b, \phi(0) \in \mathbb{F}_{4}, \phi(x):=a x^{2}+b x+\phi(0)\right\} .
$$

\footnotetext{
${ }^{14}$ W.A. Stein et al., Sage Mathematics Software (Version 4.6.2)

${ }^{15}$ The GAP Group, GAP - Groups, Algorithms, and Programming, Version 4.4.12; 2008, http://www.gap-system.org
} 
The MOG is given by a $4 \times 6$ matrix whose entries are elements of $\mathbb{F}_{2}=\{0,1\}$, and therefore provides binary words of length 24 . To check which among those words are Golay codewords, one proceeds in three steps.

1. Step 1: take a MOG configuration and calculate the parity of each 4-column of the MOG and the parity of the top row (the parity of a column or a row being the parity of the sum of its entries); they must be all equal.

2. Step 2: to each 4-column with entries $\alpha, \beta, \gamma, \delta \in \mathbb{F}_{2}$, associate the $\mathbb{F}_{4}$ element $\beta+\gamma \omega+\delta \bar{\omega}$ called its SCORE.

3. Step 3: check whether the set of six scores calculated from a given MOG form a hexacode word. If they do, then the original MOG configuration corresponds to a Golay codeword. One may take advantage of the fact that if $(a, b, c, d, e, f)$ is a hexacode word, then so are $(c, d, a, b, e, f),(a, b, e, f, c, d)$ and $(b, a, d, c, e, f)$.

For instance, the MOG configuration

\begin{tabular}{|ll|ll|ll|}
\hline 0 & 1 & 1 & 0 & 0 & 0 \\
0 & 1 & 0 & 0 & 1 & 0 \\
\hline 0 & 0 & 1 & 1 & 1 & 0 \\
0 & 0 & 0 & 1 & 0 & 0 \\
\hline
\end{tabular}

is such that all parities of columns and of top row are even, so the configuration passes Step 1. The ordered scores are $(0,1, \omega, 1, \bar{\omega}, 0)$, and one must attempt to rewrite this 6 -vector as $(a, b, \phi(0), \phi(1), \phi(\omega), \phi(\bar{\omega}))$ for a quadratic function $\phi(x)=a x^{2}+b x+\phi(0)$. In the present case, $a=0, b=1$ and $\phi(0)=\omega$, so we see that $\phi(x)=x+\omega$, and hence $\phi(1)=\bar{\omega}$, which differs from the fourth entry of the ordered scores vector. The latter is therefore not a hexacode word, and the MOG configuration does not yield a Golay codeword. The power of the MOG in this context resides in the fact that all Golay codewords can be obtained as MOG codewords. The connection between subsets of $\mathcal{I}=\{1, \ldots, 24\}$ and Golay codewords is made possible through the use of a special $4 \times 6$ array whose entries are the elements of $\mathcal{I}$, distributed in one of two ways, according to

\begin{tabular}{|rr|rr|rr|}
\hline 24 & 23 & 11 & 1 & 22 & 2 \\
3 & 19 & 4 & 20 & 18 & 10 \\
\hline 6 & 15 & 16 & 14 & 8 & 17 \\
9 & 5 & 13 & 21 & 12 & 7 \\
\hline
\end{tabular}

\begin{tabular}{|cc|cc|cc|}
\hline 23 & 24 & 1 & 11 & 2 & 22 \\
19 & 3 & 20 & 4 & 10 & 18 \\
\hline 15 & 6 & 14 & 16 & 17 & 8 \\
5 & 9 & 21 & 13 & 7 & 12 \\
$M^{\prime}$
\end{tabular}

The distribution $M$ is the original Curtis configuration, while the mirror distribution $M^{\prime}$ is due to Conway. Our labelling conventions for the codewords are compatible with the second version $M^{\prime}$, but our results could be rederived using the version $M$, provided an appropriate relabelling.

Starting with a subset of eight distinct elements of $\mathcal{I}$, one constructs a MOG configuration using $M^{\prime}$, where entries corresponding to elements in the subset are 1's and the 16 other entries are 0's. It remains to apply Steps 1 to 3 to conclude whether or not the initial set corresponds to a Golay codeword. For instance, the set $\{1,2,3,4,5,6,7,8\}$ corresponds to the MOG configuration

$\left.$\begin{tabular}{|ll|ll|ll|}
\hline 0 & 0 & 1 & 0 & 1 & 0 \\
0 & 1 & 0 & 1 & 0 & 0 \\
\hline 0 & 1 & 0 & 0 & 0 & 1 \\
1 & 0 & 0 & 0 & 1 & 0
\end{tabular}\right|$_{M^{\prime}}$,


which fails the parity test (Step 1), and therefore does not yield a Golay codeword. The same technique may be used to check whether a subset of 12 elements in $\mathcal{I}$ is a dodecad.

As an application of the MOG technique, one can check that the following twelve codewords form a basis of the Golay code:

$$
\begin{aligned}
\mathcal{O}_{1}=\{1,2,16,18,5,12,22,3\}, & \mathcal{O}_{6}=\{6,3,22,4,21,17,15,9\}, \\
\mathcal{O}_{7}=\{7,4,19,10,12,15,8,16\}, & \mathcal{O}_{8}=\{8,7,15,17,11,23,18,16\}, \\
\mathcal{O}_{16}=\{16,1,2,21,4,7,8,18\}, & \mathcal{O}_{18}=\{18,1,16,8,23,13,14,5\}, \\
\mathcal{O}_{20}=\{20,10,11,17,14,13,22,12\}, & \mathcal{O}_{23}=\{23,3,9,19,13,18,8,11\}, \\
\mathcal{O}_{24}=\{24,2,10,19,9,5,14,21\}, & \mathcal{D}_{1}=\{8,7,15,9,19,23,4,22,13,18,1,16\}, \\
\mathcal{D}_{2}=\{18,23,13,22,3,1,11,10,2,16,7,8\}, & \mathcal{D}_{3}=\{16,1,2,10,12,7,5,9,15,8,23,18\} .
\end{aligned}
$$

We now indicate how one may show that the group $\mathcal{T}_{192}$ preserves the Golay code. Act with each generator in (4.13) on a basis of the Golay code, for instance, the basis introduced in (A.2), and show that the resulting sets of eight or twelve elements correspond to Golay codewords. For instance, take the first generator from (4.13),

$$
\iota_{1}=(1,11)(2,22)(4,20)(7,12)(8,17)(10,18)(13,21)(14,16),
$$

acting on the first basis vector $\mathcal{O}_{1}$,

$$
\iota_{1}\left(\mathcal{O}_{1}\right)=\iota_{1}(\{1,2,16,18,5,12,22,3\})=\{11,22,14,10,5,7,2,3\} .
$$

The corresponding MOG configuration is

$\left.$\begin{tabular}{|ll|ll|ll|}
\hline 0 & 0 & 0 & 1 & 1 & 1 \\
0 & 1 & 0 & 0 & 1 & 0 \\
\hline 0 & 0 & 1 & 0 & 0 & 0 \\
1 & 0 & 0 & 0 & 1 & 0
\end{tabular}\right|$_{M^{\prime}}$,

which passes the parity test. Furthermore, the score vector is $(\bar{\omega}, 1, \omega, 0, \omega, 0)$ and for the MOG configuration to correspond to a Golay codeword, one needs to identify the score vector with $\left(\bar{\omega}, 1, \phi(0), \phi(1), \phi(\omega), \phi(\bar{\omega})\right.$ where $\phi(x)=\bar{\omega} x^{2}+x+\omega$. Since $\phi(0)=\omega, \phi(1)=\bar{\omega}+$ $1+\omega=0, \phi(\omega)=\bar{\omega} \omega^{2}+\omega+\omega=\omega$ and $\phi(\bar{\omega})=\bar{\omega}^{3}+\bar{\omega}+\omega=0$, we are through: the set $\iota_{1}(\{1,2,16,18,5,12,22,3\})$ is an octad.

A related technique used in this work consists in constructing the unique octad associated with 5 given elements of $\mathcal{I}$ via the MOG. Suppose we choose the set $A=\{3,6,14,17,18\}$ and wish to complete $A$ so that one obtains an octad. First, one constructs a MOG start configuration where one replaces the elements belonging to $A$ by 1 , and all elements in $\mathcal{I} \backslash A$ by nothing in the Conway MOG array $M^{\prime}$ of (A.1),

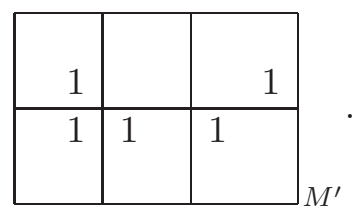

Then one observes that, were all the blanks replaced by 0's, 3 columns would have odd parity, and 3 would have even parity, while the top row also would have even parity. One has three extra entries of 1 to distribute in such a way that the configuration passes the parity test. If the solution corresponds to odd parity, columns 3,5 and 6 cannot accommodate more entries 
of 1 , so the score vector is partially known and reads $(a, b, \omega, \phi(1), \omega, 1)$, with $a, b \in \mathbb{F}_{4}$ and $\phi(x)=a x^{2}+b x+c$. So $\phi(0)=c=\omega$ and the system $\phi(\omega)=a \bar{\omega}+b \omega+\omega=\omega ; \phi(\bar{\omega})=$ $a \omega+b \bar{\omega}+\omega=1$ has no solution for $a, b \in \mathbb{F}_{4}$. One thus tries a solution corresponding to even parity. In this case, columns 1,2 and 4 cannot accommodate more entries of 1 if one hopes to pass the parity test. The partial score is $(0, \bar{\omega}, \omega+n, 0, \phi(\omega), \phi(\bar{\omega}))$, with $\phi(x)=\bar{\omega} x+\omega+n$ for $n=0,1$ or $\bar{\omega}$. The equation $\phi(1)=\bar{\omega}+\omega+n=0$ implies that $n=1$. Thus $\phi(\omega)=1+\omega+1=\omega$ and $\phi(\bar{\omega})=\omega+\omega+1=1$. The reconstructed hexacode word is $(0, \bar{\omega}, \bar{\omega}, 0, \omega, 1)$ and the octad MOG configuration is thus,

$\left.\begin{array}{|ll|ll|ll|}\hline 0 & 0 & 0 & 0 & 1 & 1 \\ 0 & 1 & 1 & 0 & 0 & 1 \\ \hline 0 & 1 & 1 & 0 & 1 & 0 \\ 0 & 0 & 0 & 0 & 0 & 0\end{array}\right]_{M^{\prime}}$.

In other words, the unique octad formed from the partial knowledge encoded in the set $\{3,6,14,17,18\}$ is given by $\{2,3,6,14,17,18,20,22\}$.

\section{B Tables}

The following tables provide a roadmap through our notations. They list the relevant lattices that we introduce in sections 2 and 3 and which we use throughout this work. As a shorthand we introduce the following notation: Consider an even unimodular lattice $\Gamma$. To state that $\Gamma$ can be obtained by the gluing construction of proposition 2.1.1 from a pair of primitive sublattices $\Lambda, \mathcal{V} \subset \Gamma$, where $\Lambda$ and $\mathcal{V}$ are orthogonal complements of one another in $\Gamma$, we write $\Gamma=\Lambda \bowtie \mathcal{V}$. 


\begin{tabular}{|c|c|c|c|c|c|c|}
\hline rank & $\begin{array}{l}\text { sign. \& } \\
\text { disc. grp. }\end{array}$ & lattice & $\begin{array}{c}\text { ref. } \\
\text { in text }\end{array}$ & $\begin{array}{l}\text { sign. \& } \\
\text { disc. grp. }\end{array}$ & lattice & $\begin{array}{c}\text { ref. } \\
\text { in text }\end{array}$ \\
\hline 24 & $\begin{array}{l}(4,20) \\
\{\mathrm{id}\}\end{array}$ & $\begin{array}{l}H_{*}(X, \mathbb{Z})=H_{0}(X, \mathbb{Z}) \oplus H_{2}(X, \mathbb{Z}) \oplus H_{4}(X, \mathbb{Z}) \\
\cong U^{4} \oplus E_{8}^{2}(-1) \\
\text { full integral homology lattice of K3 surface } X \\
\qquad H_{*}(X, \mathbb{Z})=\mathcal{K} \bowtie \mathcal{P}\end{array}$ & Prop. 2.2.4 & $\begin{array}{l}(24,0) \\
\{\mathrm{id}\}\end{array}$ & $\begin{array}{l}N=\left\{\nu \in\left(A_{1}^{24}\right)^{*} \mid \bar{\nu} \in \mathcal{G}_{24}\right\} \\
\text { Niemeier lattice of type } A_{1}^{24} \\
\quad N=\widetilde{K} \bowtie \widetilde{\Pi}=\widetilde{\mathcal{K}}_{n_{0}} \bowtie \widetilde{\mathcal{P}}_{n_{0}}\end{array}$ & $\begin{array}{l}\text { Prop. } 2.3 .1 \\
\text { Prop. } 2.3 .6\end{array}$ \\
\hline 22 & $\begin{array}{l}(3,19) \\
\{\mathrm{id}\} \\
\end{array}$ & $\begin{array}{c}H_{2}(X, \mathbb{Z}) \cong U^{3} \oplus E_{8}^{2}(-1) \\
=K \bowtie \Pi \\
\text { K3-lattice }\end{array}$ & $\begin{array}{c}\text { after } \\
\text { Def. } 2.2 .1 \\
\text { before } 2.6\end{array}$ & & & \\
\hline 17 & $\begin{array}{l}(0,17) \\
\left(\mathbb{Z}_{2}\right)^{7}\end{array}$ & $\begin{array}{l}\qquad \mathcal{P}:=\Pi \oplus \operatorname{span}_{\mathbb{Z}}\left\{v_{0}-v\right\} \\
\text { primitive sublattice of } H_{*}(X, \mathbb{Z})\end{array}$ & Prop. 2.2.4 & $\begin{array}{l}(17,0) \\
\left(\mathbb{Z}_{2}\right)^{7}\end{array}$ & $\begin{array}{c}\widetilde{\mathcal{P}}_{n_{0}}=\widetilde{\Pi} \oplus \operatorname{span}_{\mathbb{Z}}\left\{f_{n_{0}}\right\} \cong \mathcal{P}(-1) \\
\text { primitive sublattice of } N\end{array}$ & Prop. 2.3.6 \\
\hline 16 & $\begin{array}{l}(0,16) \\
\left(\mathbb{Z}_{2}\right)^{6}\end{array}$ & $\begin{array}{c}\Pi=\operatorname{span}_{\mathbb{Z}}\left\{E_{\vec{a}}, \vec{a} \in \mathbb{F}_{2}^{4}, \frac{1}{2} \sum_{\vec{a} \in H_{i}} E_{\vec{a}}\right\} \\
H_{i}, i=1, . ., 5 \text { affine hyperplanes in } \mathbb{F}_{2}^{4} \\
\text { Kummer lattice; primitive sublattice of } H_{2}(X, \mathbb{Z})\end{array}$ & Prop. 2.2 .3 & $\begin{array}{l}(16,0) \\
\left(\mathbb{Z}_{2}\right)^{6}\end{array}$ & $\begin{array}{c}\widetilde{\Pi} \cong \Pi(-1) \\
\widetilde{\Pi}=\operatorname{span}_{\mathbb{Z}}\left\{f_{n}, n \notin \mathcal{O}_{9}, \frac{1}{2} \sum_{n \in \mathcal{H}_{i}} f_{n}\right\} \\
\mathcal{H}_{i}, i=1, . ., 5 \text { octads, } \mathcal{H}_{i} \cap \mathcal{O}_{9}=\emptyset \\
\text { primitive sublattice of } N\end{array}$ & $\begin{array}{l}\text { Prop. } 2.3 .4 \\
\begin{array}{l}2.13 \\
\text { Prop. } 2.3 .3\end{array}\end{array}$ \\
\hline 8 & & & & $\begin{array}{l}(8,0) \\
\left(\mathbb{Z}_{2}\right)^{6}\end{array}$ & $\begin{aligned} \widetilde{K}= & \operatorname{span}_{\mathbb{Z}}\left\{f_{n}, n \in \mathcal{O}_{9}, \frac{1}{2} \sum_{n \in \mathcal{O}_{9}} f_{n}\right\} \\
& \text { primitive sublattice of } N\end{aligned}$ & Prop. 2.3.4 \\
\hline 7 & $\begin{array}{l}(4,3) \\
\left(\mathbb{Z}_{2}\right)^{7}\end{array}$ & $\begin{array}{c}\mathcal{K}:=\mathcal{P}^{\perp} \cap H_{*}(X, \mathbb{Z}) \\
=K \oplus \operatorname{span}_{\mathbb{Z}}\left\{v_{0}+v\right\} \\
\text { primitive sublattice of } H_{*}(X, \mathbb{Z})\end{array}$ & Prop. 2.2.4 & $\begin{array}{l}(7,0) \\
\left(\mathbb{Z}_{2}\right)^{7}\end{array}$ & $\begin{array}{c}\widetilde{\mathcal{K}}_{n_{0}}:=\widetilde{\mathcal{P}}_{n_{0}}^{\perp} \cap N \\
=\operatorname{span}_{\mathbb{Z}}\left\{f_{n}, n \in \mathcal{O}_{9} \backslash\left\{n_{0}\right\}\right\} \\
\operatorname{primitive~sublattice~of~} N^{\text {primitive }}\end{array}$ & Prop. 2.3.6 \\
\hline 6 & $\begin{array}{l}(3,3) \\
\left(\mathbb{Z}_{2}\right)^{6}\end{array}$ & 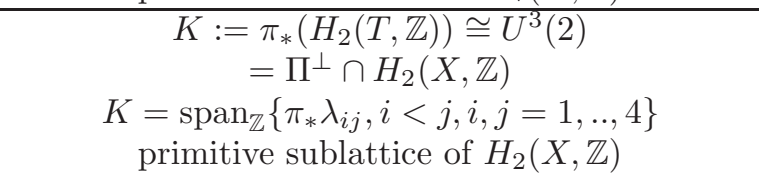 & $\begin{array}{l}\text { Prop. 2.2.3 } \\
\text { after 2.8 }\end{array}$ & & & \\
\hline 2 & $\begin{array}{l}(1,1) \\
\{\text { id }\}\end{array}$ & $\begin{array}{c}H_{0}(X, \mathbb{Z}) \oplus H_{4}(X, \mathbb{Z}) \cong U \\
\text { hyperbolic lattice }\end{array}$ & 2.3 & & & \\
\hline
\end{tabular}

Table 1: Notations for lattices introduced in section 2 and used throughout the text. 


\begin{tabular}{|c|c|c|}
\hline signature & lattice & Ref. in text \\
\hline $\begin{array}{c}\left(4, m_{-}\right), \\
m_{-} \geq 1\end{array}$ & $\begin{array}{c}L^{G} \text { sublattice of } H_{*}(X, \mathbb{Z}) \text { invariant } \\
\text { under a group } G \text { of symplectic } \\
\text { automorphisms preserving the } \\
\text { dual Kähler class }\end{array}$ & Prop. 3.2.4 \\
\hline $\begin{array}{c}\left(0,20-m_{-}\right), \\
m_{-} \geq 1\end{array}$ & $L_{G}=\left(L^{G}\right)^{\perp} \cap H_{*}(X, \mathbb{Z})$ & Prop. 3.2.4 \\
\hline $\begin{array}{c}\left(0,22-l_{-}\right), \\
l_{-} \geq 2\end{array}$ & $M_{G}:=L_{G} \oplus \operatorname{span}_{\mathbb{Z}}\left\{e, v_{0}-v\right\}$ & Prop. 3.3.6 \\
\hline $\begin{array}{c}\left(4, l_{-}-2\right), \\
l_{-} \geq 2\end{array}$ & $\begin{array}{c}M_{G}^{\prime}:=\pi_{*}\left(\left(H_{2}(T, \mathbb{Z})\right)^{G_{T}^{\prime}}\right) \oplus \operatorname{span}_{\mathbb{Z}}\left\{v_{0}+v\right\} \\
=M_{G}^{\perp} \cap H_{*}(X, \mathbb{Z})\end{array}$ & Prop. 3.3.6 \\
\hline $\begin{array}{c}\Pi^{G}:=\Pi \cap H_{*}(X, \mathbb{Z})^{G} \\
=\operatorname{span}_{\mathbb{Z}}\{e\}\end{array}$ & Prop. 3.3.6 \\
\hline
\end{tabular}

Table 2: Notations for lattices introduced in section 3 and used throughout the text. $G$ denotes the group of dual Kähler class preserving symplectic automorphisms of a Kummer surface $X$ with complex structure and dual Kähler class induced from the underlying torus $T$. The group of non-translational holomorphic symplectic automorphisms of $T$ is $G_{T}^{\prime}$.

\section{References}

[1] T. Eguchi, H. Ooguri, And Y. Tachikawa, Notes on the K3 surface and the Mathieu group $M_{24}$, Exper. Math. 20 (2011) 91 arXiv:1004.0956].

[2] O. Alvarez, T.P. Killingback, M. Mangano, and P. Windey, String theory and loop space index theorems, Commun. Math. Phys. 111 (1987), 1.

[3] E. Witten, Elliptic genera and quantum field theory, Commun. Math. Phys. 109 (1987) 525 .

[4] T. Eguchi, H. Ooguri, A. Taormina, And S.-K. Yang, Superconformal algebras and string compactification on manifolds with $S U(n)$ holonomy, Nucl. Phys. B315 (1989) 193.

[5] M.C.N. Cheng, K3 surfaces, $N=4$ dyons, and the Mathieu group $M_{24}$, Commun. Number Theory Phys. 4 (2010), 623 [arXiv:1005.5415].

[6] M.R. Gaberdiel, S. Hohenegger, and R. Volpato, Mathieu moonshine in the elliptic genus of K3, JHEP 1010 (2010) 062 [arXiv:1008.3778]. 
[7] M.R. Gaberdiel, S. Hohenegger, and R. Volpato, Mathieu twining characters for K3, JHEP 1009 (2010) 058 arXiv:1006.0221].

[8] S. Govindarajan, Brewing moonshine for Mathieu, arXiv:1012.5731,

[9] S. MukaI, Finite groups of automorphisms of K3 surfaces and the Mathieu group, Invent. Math. 94 (1988) 183.

[10] S. Kondo, Niemeier lattices, Mathieu groups and finite groups of symplectic automorphisms of K3 surfaces, Duke Math. J. 92 (1998) 593, Appendix by S. Mukai.

[11] V.V. Nikulin, Finite automorphism groups of Kaehler K3 surfaces, Trans. Mosc. Math. Soc. 38 (1980) 71.

[12] V.V. Nikulin, Integral symmetric bilinear forms and some of their applications, Math. USSR Isv. 14 (1980) 103.

[13] E. Witt, Eine Identität zwischen Modulformen zweiten Grades, Abh. math. Sem. Univ. Hamburg 14 (1941) 323.

[14] M. Kneser, Klassenzahlen definiter quadratischer Formen, Arch. Math. 8 (1957) 241.

[15] M.R. Gaberdiel, S. Hohenegger, and R. Volpato, Symmetries of K3 sigma models, Commun. Num. Theor. Phys. 6 (2012)1 [arXiv:1106.4315].

[16] D.R. Morrison, On K3 surfaces with large Picard number, Invent. Math. 75 (1984) 105.

[17] J. Milnor, On simply connected 4-manifolds, in: Symposium Internacional de Topologia Algebraica, La Universidad Nacional Autónoma de México y la UNESCO, 1958, pp. 122 128.

[18] J.-P. Serre, Cours d'arithmétique, Collection SUP: 'Le Mathématicien', vol. 2, Presses Universitaires de France, Paris, 1970.

[19] J. Milnor And D. Husemoller, Symmetric bilinear forms, Springer-Verlag, 1973.

[20] H.V. Niemeier, Definite quadratische Formen der Dimension 24 und Diskriminante 1, J. Number Theory 5 (1973) 142.

[21] I.I. PJATECKII-ŠAPIRO AND I. R. ŠAfAREvič, Torelli's theorem for algebraic surfaces of type K3, Izv. Akad. Nauk SSSR Ser. Mat. 35 (1971) 530.

[22] V.V. Nikulin, On Kummer surfaces, Math. USSR Isv. 9 (1975) 261.

[23] K. Kodaira, On the structure of compact complex analytic surfaces, I. Am. J. Math. 86 (1964) 751.

[24] I.R. ŠAfarevič, Algebraic surfaces, in: Proceedings of the Steklov Institute of Mathematics, No. 75 (1965), K. Kodaira and D.C. Spencer, eds., American Mathematical Society, Providence, R.I., 1965, pp. ix+281, Translated from Russian by Susan Walker.

[25] J.H. Conway and N.J.A. Sloane, Sphere Packings, Lattices and Groups, SpringerVerlag, New York Berlin Heidelberg London Paris Tokyo, 1988.

[26] V.V. Nikulin, Kahlerian K3 surfaces and Niemeier lattices, arXiv:1109.2879.

[27] J.A. ToDD, On representations of the Mathieu groups as collineation groups, J. London Math. Soc. 34 (1959) 406. 
[28] J.A. TodD, A representation of the Mathieu group $M_{24}$ as a collineation group, Ann. Mat. Pura Appl. (4) 71 (1966) 199.

[29] J.H. Conway, Three lectures on exceptional groups, in: Finite Simple Groups, Proceedings of an instructional conference organized by the London Mathematical Society (London and New York) (M.B. Powell and G. Higman, eds.), Academic Press (1971).

[30] C. Jordan, Traité des substitutions et des équations algébriques, Paris (1870).

[31] R. T. CuRTis, Further elementary techniques using the miracle octad generator, Proc. Edinburgh Math. Soc. 32 (1989) 345.

[32] V.S. Kulikov, Surjectivity of the period mapping for K3 surfaces, Uspehi Mat. Nauk 32 (1977), no. 4(196) 257.

[33] E. Looijenga, A Torelli theorem for Kähler-Einstein K3 surfaces, vol. 894 of Lecture Notes in Math., Springer, Berlin, 1981, pp. 107-112.

[34] Y. NAmikawa, Surjectivity of period map for K3 surfaces, in: Classification of algebraic and analytic manifolds (Katata, 1982), vol. 39 of Progr. Math., Birkhäuser Boston, Boston, MA, 1983, pp. 379-397.

[35] Y.T. Siu, A simple proof of the surjectivity of the period map of K3 surfaces, Manuscripta Math. 35 (1981) no. 3, 311.

[36] A.N. Todorov, Applications of the Kähler-Einstein-Calabi-Yau metric to moduli of K3 surfaces, Invent. Math. 61 (1980) no. 3, 251.

[37] M.F. Atiyah, On analytic surfaces with double points, Proc. Roy. Soc. London Ser. A247 (1958) 237.

[38] T. Shioda And H. Inose, On singular K3 surfaces, in: Complex Analysis and Algebraic Geometry, W.L. Bailey and T. Shioda, eds., Cambridge Univ. Press, 1977, pp. 119-136.

[39] T. ShiodA, On elliptic modular surfaces, J. Math. Soc. Japan 24 (1972) 20.

[40] I.T. Todonov, How many Kähler metrics has a K3 surface?, in: Arithmetic and Geometry: Papers dedicated to I.R.Shafarevich on the occasion of his sixtieth birthday, vol. 2, Birkhäuser, 1983, pp. 451-463.

[41] P. Griffiths And J. Harris, Principles of algebraic geometry, John Wiley \& Sons, New York, 1978.

[42] P.S. Aspinwall and D.R. Morrison, String theory on K3 surfaces, in: Mirror symmetry II, B. Greene and S.T. Yau, eds., AMS, 1994, pp. 703-716 hep-th/9404151].

[43] W. Nahm and K. Wendland, A hiker's guide to $K 3$ - Aspects of $N=(4,4)$ superconformal field theory with central charge $c=6$, Commun. Math. Phys. 216 (2001) 85 hep-th/9912067.

[44] W. Barth, C. Peters, And A. Van de Ven, Compact Complex Surfaces, SpringerVerlag, Berlin Heidelberg New York Tokyo, 1984.

[45] D. Mumford, The topology of normal singularities of an algebraic surface and a criterion for simplicity, Inst. Hautes Études Sci. Publ. Math. No. 9 (1962), 5.

[46] G. XIaO, Galois covers between K3 surfaces, Ann. Inst. Fourier (Grenoble) 46 (1996) 73. 
[47] G. Mason, Symplectic automorphisms of K3-Surfaces (after S. Mukai and V.V. Nikulin), CWI Newslett. 13 (1986) 3.

[48] A. FujIKI, Finite automorphism groups of complex tori of dimension two, Publ. Res. Inst. Math. Sci. 24 (1988) no. 1, 1.

[49] R. T. CuRTis, Symmetric generation of groups, Encyclopedia of Mathematics, Cambridge University Press, 2007.

[50] K. Wendland, Moduli spaces of unitary conformal field theories, Ph.D. thesis, University of Bonn, 2000.

[51] A. TaOrmina And K. Wendland, Symmetry-surfing the moduli space of Kummer K3s, arXiv:1303.2931.

[52] R. T. Curtis, A new combinatorial approach to $M_{24}$, Math. Proc. Camb. Phil. Soc. 79 (1976), 25-42. 